\title{
WestVirginiaUniversity
}

THE RESEARCH REPOSITORY @ WVU

Graduate Theses, Dissertations, and Problem Reports

1999

\section{Efficient and flexible algorithm for plant layout generation}

Li Weng

West Virginia University

Follow this and additional works at: https://researchrepository.wvu.edu/etd

\section{Recommended Citation}

Weng, Li, "Efficient and flexible algorithm for plant layout generation" (1999). Graduate Theses,

Dissertations, and Problem Reports. 2301.

https://researchrepository.wvu.edu/etd/2301

This Dissertation is protected by copyright and/or related rights. It has been brought to you by the The Research Repository @ WVU with permission from the rights-holder(s). You are free to use this Dissertation in any way that is permitted by the copyright and related rights legislation that applies to your use. For other uses you must obtain permission from the rights-holder(s) directly, unless additional rights are indicated by a Creative Commons license in the record and/ or on the work itself. This Dissertation has been accepted for inclusion in WVU Graduate Theses, Dissertations, and Problem Reports collection by an authorized administrator of The Research Repository @ WVU.

For more information, please contact researchrepository@mail.wvu.edu. 


\title{
EFFICIENT AND FLEXIBLE ALGORITHM FOR PLANT LAYOUT GENERATION
}

\author{
Li Weng
}

\section{DISSERTATION}

\author{
Submitted to \\ The College of Engineering and Mineral Resources \\ at \\ West Virginia University \\ In partial fulfillment of the requirements \\ for the degree of
}

Doctor of Philosophy

in

Industrial Engineering

B. Gopalakrishnan, Ph.D., Chair

Hany H. Ammar, Ph.D.

Keh-Minn Chang, Ph.D.

Majid Jaraiedi, Ph.D.

Ralph W. Plummer, Ph.D.

Department of Industrial and Management System Engineering

Morgantown, West Virginia

1999

Keywords: Plant Layout, Facilities Planning, Computer-Aided Layout Copyright 1999 Li Weng 


\title{
ABSTRACT \\ EFFICIENT AND FLEXIBLE ALGORITHM FOR PLANT LAYOUT GENERATION
}

\author{
Li Weng
}

A facilities layout, also called plant layout, consists of the production areas, production related or support areas and personnel areas within the building. Plant layout design is one of the strategic fields that determine the long run efficiency of operation.

This dissertation proposes an efficient and flexible plant layout algorithm to minimize the material handling cost and deal with change in future. A material flow forecasting tool, a scheduling module related to layout design and an evaluation method of flexible layouts are also proposed by this dissertation. A computer-based system will be developed to integrate all of the functions.

The first chapter of this paper introduces the issues in plant layout design. Because the volume and the mix of products to be produced are typically not known with certainty nor are they static over time, it is desirable to design a flexible layout to accommodate these changes. Chapter two gives a brief review of various algorithms and programs of layout design. Chapter three provides insight into the approach taken for the proposed flexible layout design. Then it presents all major algorithms involved in forecasting, pair exchange layout design, flexible layout design and evaluation. Chapter four is concerned with the computer-based system analysis and design. Chapter five uses several case studies to validate the algorithms and computer system. Chapter six explains the contributions of this research. Chapter seven gives the conclusions and future work. 


\section{ACKNOWLEDGEMENT}

I would like to express my sincere gratitude to my research advisor, Dr. B.

Gopalakrishnan, for his invaluable guidance, support and constant encouragement throughout the length of this research work at West Virginia University. I would also like to thank my committee members, Dr. Ralph W. Plummer, Dr. Majid Jaraiedi, Dr. Keh-Minn Chang and Dr. Hany H. Ammar, for their advice and assistance. 


\section{TABLE OF CONTENTS}

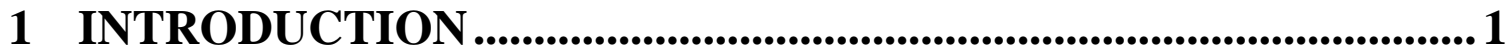

1.1 Concept of Facilities Planning and Plant Layout ............................... 1

1.2 ImPortance of Plant Layout ............................................................ 2

1.3 OBJECTIVES OF THE PLANT LAYOUT STRATEGY …................................... 2

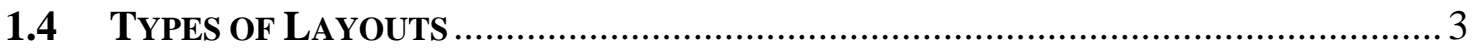

1.5 Layout Design Methods and Computer PaCkageS................................ 3

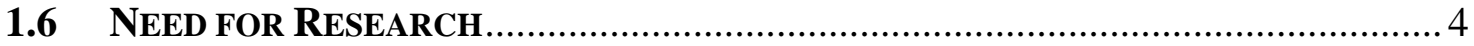

1.6.1 Department Shape and Area ........................................................................... 5

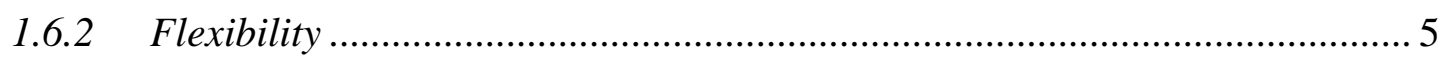

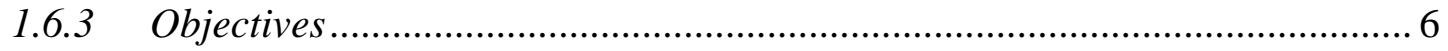

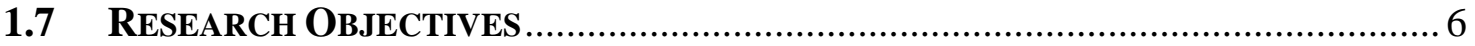

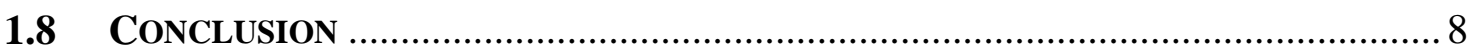

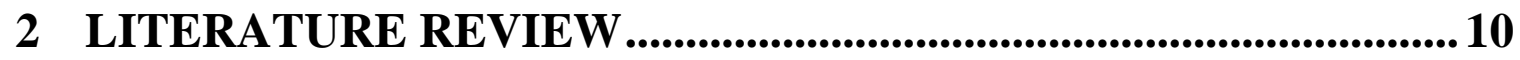

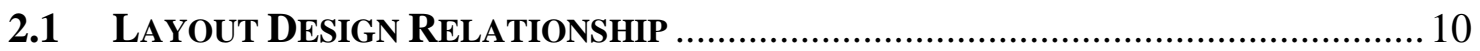

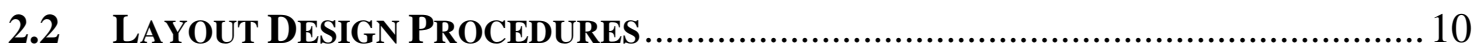

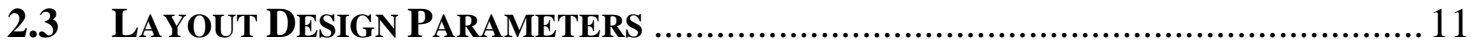

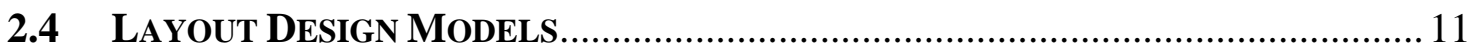

2.5 Computer-Aided Layout Design SofTWARE ANd CoMPANIES ................. 13

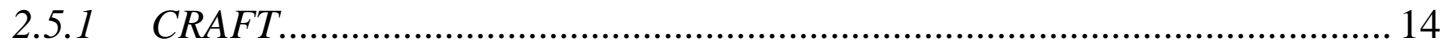

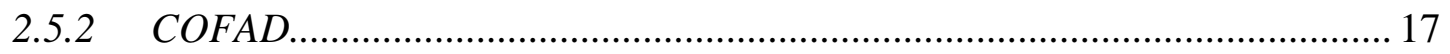

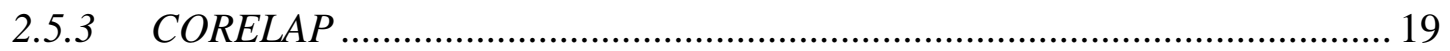

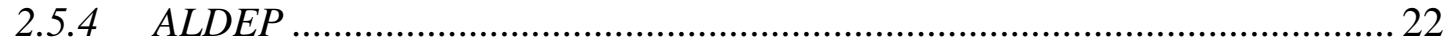

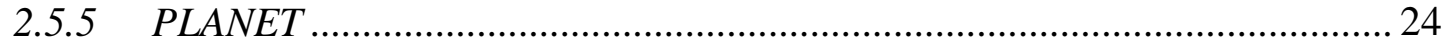

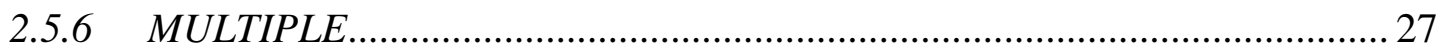




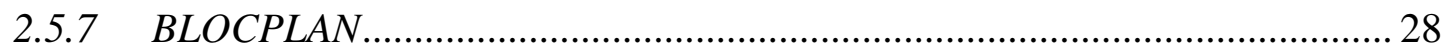

2.5.8 SHAPE

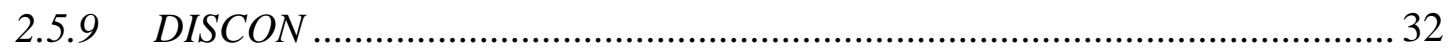

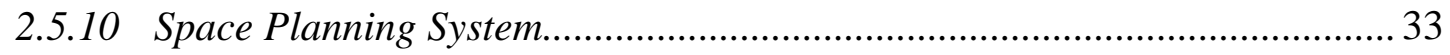

2.6 CuRrent Flexible Layout Design ReSEarCh.......................................... 34

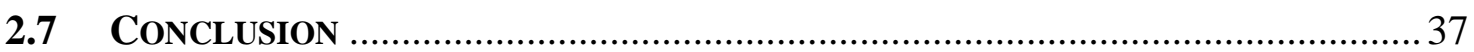

\section{RESEARCH APPROACH AND ALGORITHMS ..........................38}

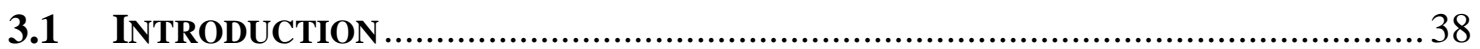

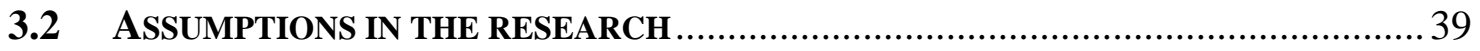

3.3 FLEXIBLE LAYOUT DESIGN SYSTEM …………...........................................

3.4 Computer Program Data Flow Diagram ………………...................... 42

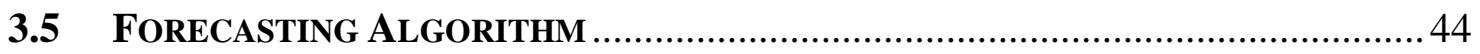

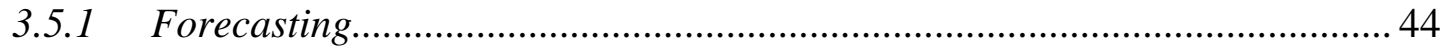

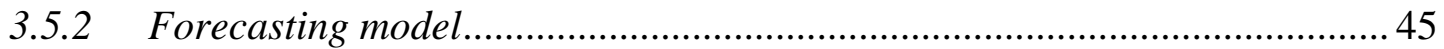

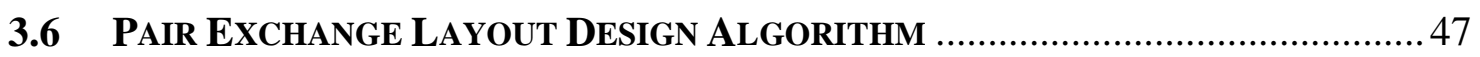

3.7 FLEXIBLE LAYOUT DESIGN ALgORITHM ...................................................... 49

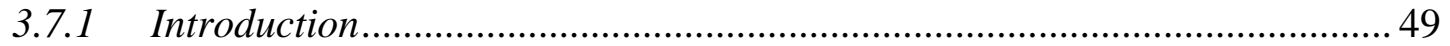

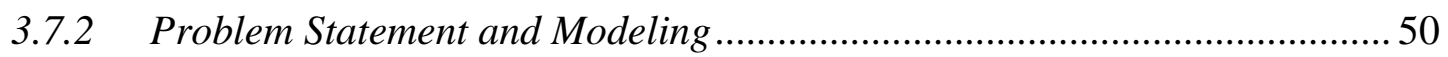

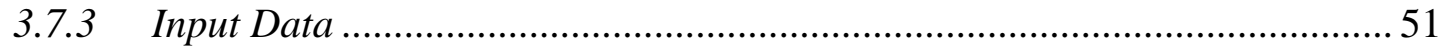

3.7.4 Flexible Layout Design Procedures......................................................... 51

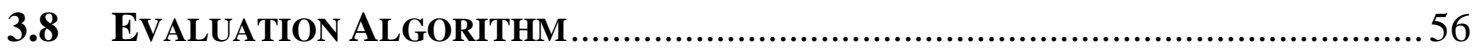

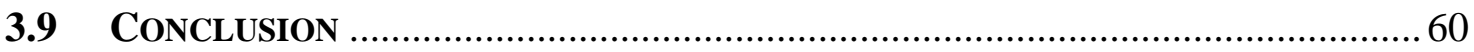

\section{COMPUTER PROGRAM DESIGN ....................................................... 61}

4.1 OVERALL PROGRAM STRUCTURE..........................................................................61

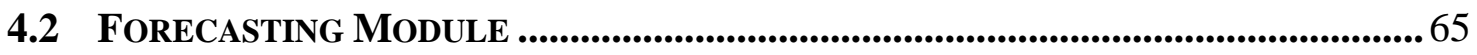

4.3 Initialization Module and Pair Exchange Layout Design Module .... 67

4.4 Flexible Layout DeSign Module .........................................................................69 
4.5 RESCHEDULE_FLOW MODULE ............................................................................... 74

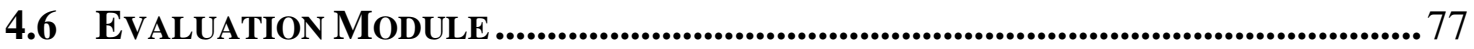

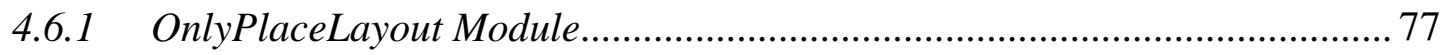

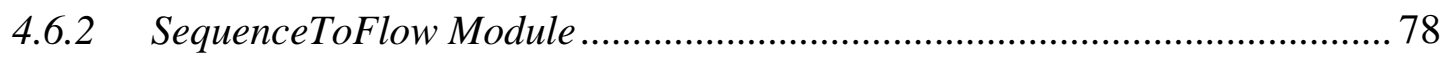

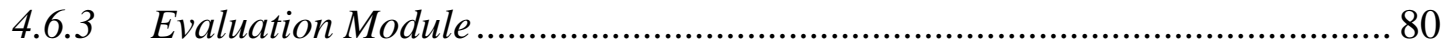

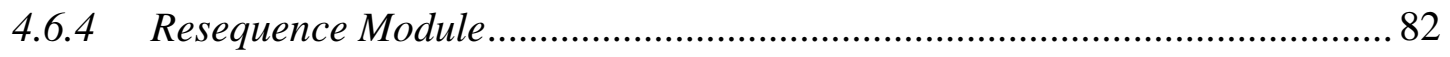

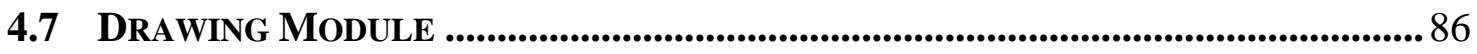

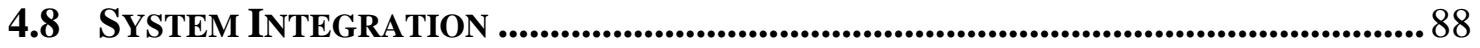

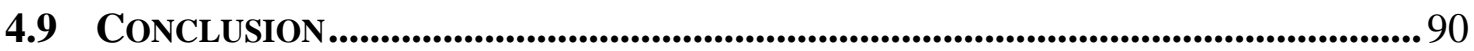

\section{SYSTEM IMPLEMENTATION AND VALIDATION ................... 91}

5.1 SYSTEM IMPLEMENTATION ........................................................................ 91

5.2 Deterministic Material Flow CASE STUDY ................................................ 91

5.3 VALIDATION OF LiTERATURE DATA ...................................................................96

5.3.1 Flexible Layout Design Method and Comparison to CRAFT Method.......... 99

5.3.2 Comparison to CORELAP Method .............................................................. 104

5.3.3 Comparison to ALDEP Method ............................................................. 106

5.3.4 Comparison to Some Publishing ............................................................ 108

5.4 ForeCASTING VALIDATION ................................................................ 110

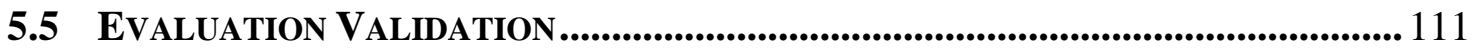

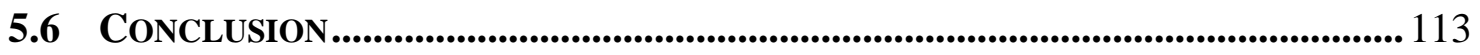

\section{CONTRIBUTIONS OF THE RESEARCH ................................ 114}

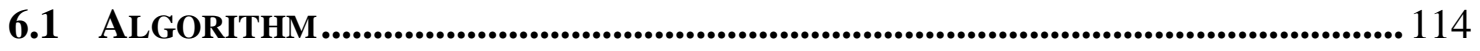

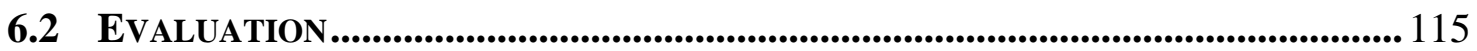

6.3 INTEGRATION........................................................................................................ 116

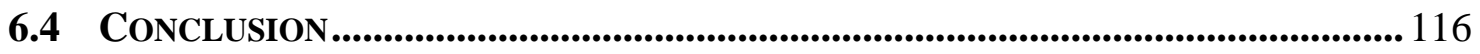

7 CONCLUSIONS AND FUTURE WORK ....................................117 


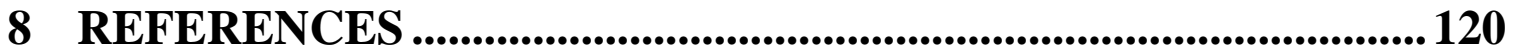

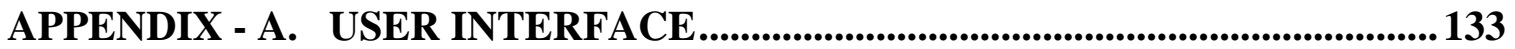

APPENDIX - B. RELATIONSHIPS OF TABLES/QUERIES.................................... 134

APPENDIX - C. LIST OF ENTITIES .......................................................................... 135

APPENDIX - D. ENTITY INTERACTIONS.................................................................139 


\section{LIST OF FIGURES}

Figure 2-1 Relationship between Product, Process, Schedule and Layout Design......... 10

Figure 2-2 Systematic Layout Planning (SLP) Procedure [Muther, 1973] ..................... 12

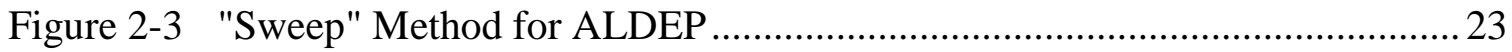

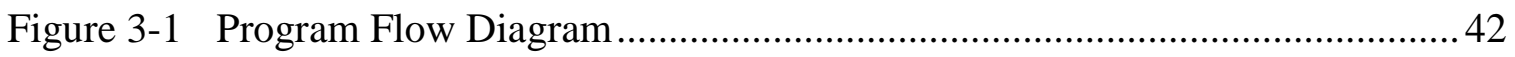

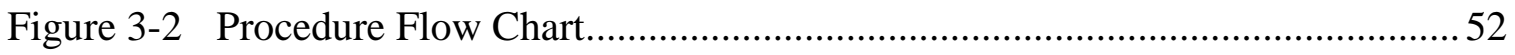

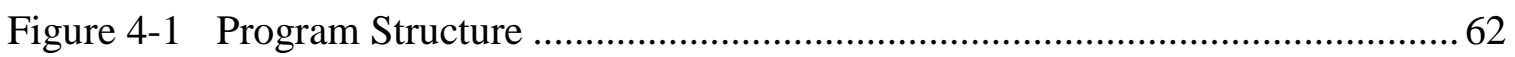

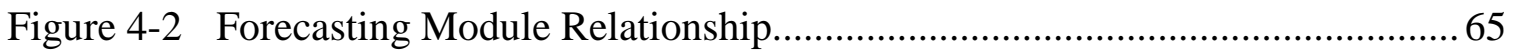

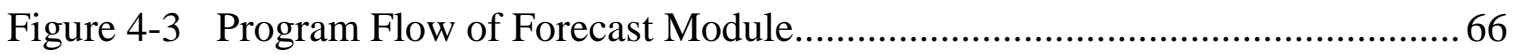

Figure 4-4 Pair Exchange Layout Design Module Relationship ................................. 68

Figure 4-5 Program Flow of Module PairExchange ............................................. 70

Figure 4-6 Flexible Layout Design Module Relationships ...................................... 72

Figure 4-7 Program Flow of Module Split_dept.................................................. 73

Figure 4-8 Reschedule Module Relationships ...................................................... 75

Figure 4-9 Program Flow of Module Reschedule_flow ............................................ 76

Figure 4-10 OnlyPlaceLayout Module Relationship ............................................... 78

Figure 4-11 The Program Flow of Module OnlyPlaceLayout ......................................... 79

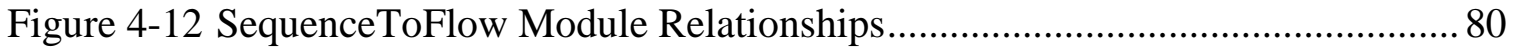

Figure 4-13 Program Flow of Module SequenceToFlow ........................................... 81

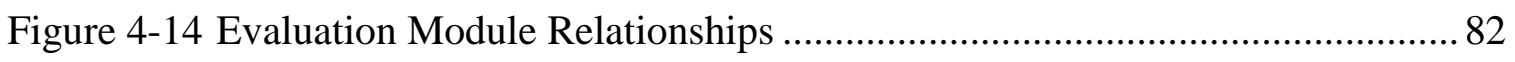

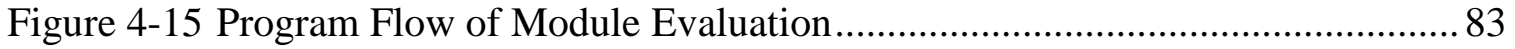

Figure 4-16 Resequence Module Relationships...................................................... 84

Figure 4-17 Program Flow of Module Resequence ................................................. 85

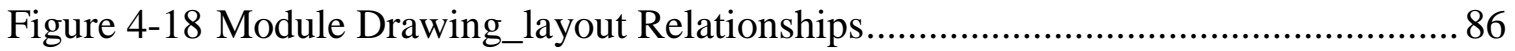

Figure 4-19 Program Flow of Module Drawing ..................................................... 87

Figure 5-1 Layout After Department J Is Split ..................................................... 93

Figure 5-2 Layout After Department L Is Split ................................................... 95

Figure 5-3 Layout After Department D Is Split ................................................ 96 


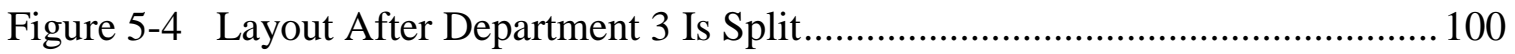

Figure 5-5 Layout After Department 1 Is Split...................................................... 102

Figure 5-6 Layout After Department 6 is Split....................................................... 103

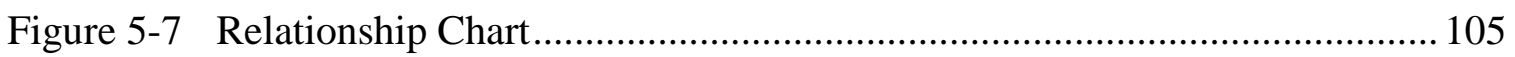

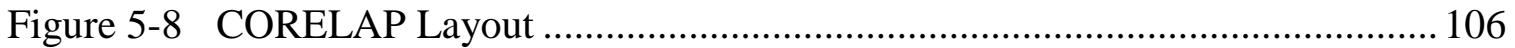

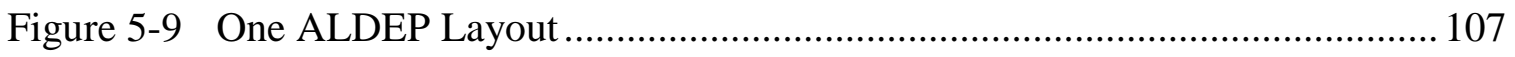

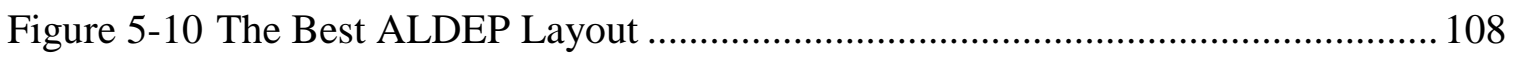




\section{LIST OF TABLES}

Table 5-1 Material Flows Chart........................................................................ 92

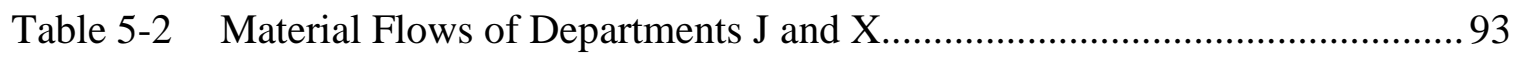

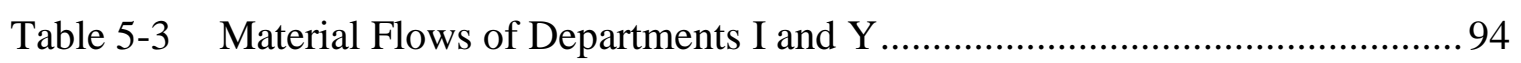

Table 5-4 Material Flows of Departments D and Z ............................................... 95

Table 5-5 Five Possible Material Flow Charts ........................................................ 97

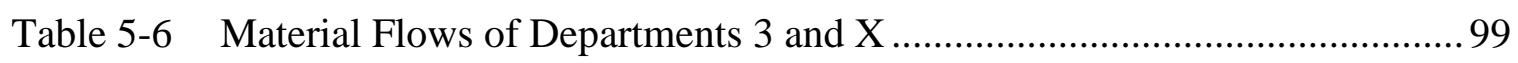

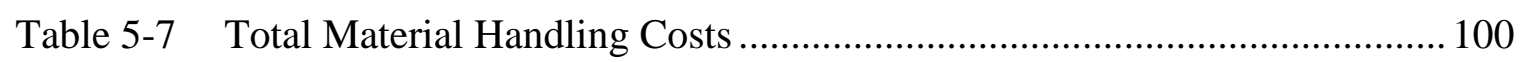

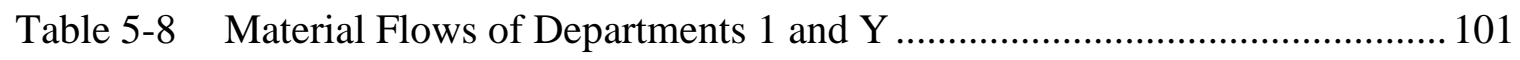

Table 5-9 Total Material Handling Costs ............................................................... 102

Table 5-10 Material Flows of Departments 6 and Z .............................................. 103

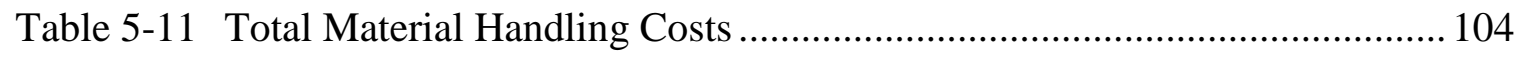

Table 5-12 ALDEP Layouts and Material Handling Costs ........................................ 107

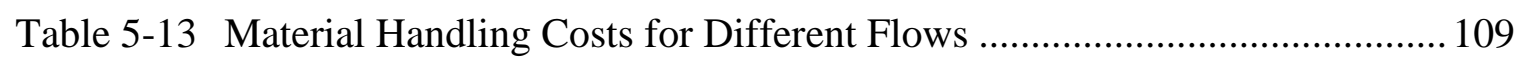

Table 5-14 Expected Material Handling Costs for Different Probabilities ................... 110

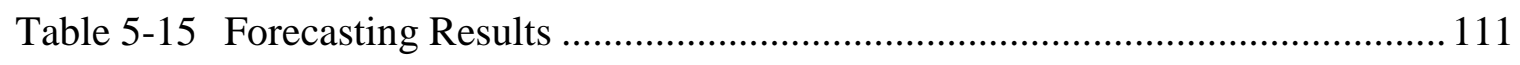

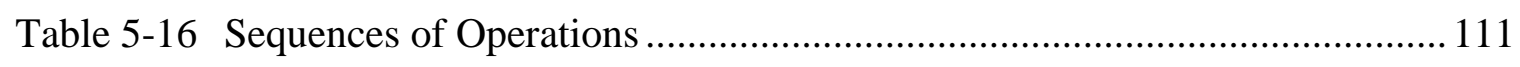

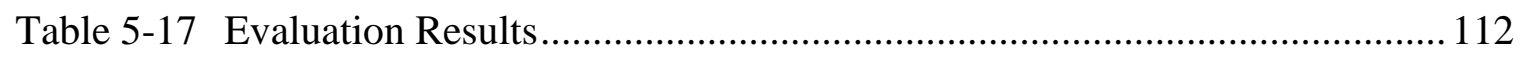




\section{INTRODUCTION}

\subsection{Concept of Facilities Planning and Plant Layout}

Facilities planning is a complex and broad subject that covers several disciplines. It involves civil, electrical, industrial and mechanical engineers, as well as architects, consultants, managers and urban planners.

According to Tompkins and White [Tompkins, 1996], facilities planning determines how an activity's tangible fixed assets best support achieving the activity's objective. Facilities planning can be divided into two components: facilities location and facilities design.

Facilities location is about placement of the facility on a specific plot of land with respect to customers, suppliers and other facilities. Facilities design consists of the facility systems design, the layout design and the handling systems design. The facility systems consist of the structural systems, the environmental systems, the lighting/electrical systems and safety systems. The layout consists of all equipment, machinery and furnishings within the building structure. The handling system consists of the mechanisms needed to satisfy the required facility interactions.

For a manufacturing plant, the facilities layout, also called plant layout, consists of the production areas, production related or support areas and personnel areas within the building. 


\subsection{Importance of Plant Layout}

Plant layout problems can occur in a large number of ways and can have significant effects on the overall effectiveness of the production system. According to Tompkins [Tompkins, 1996], since 1955, approximately $8 \%$ of the Gross National Product (GNP) has been spent annually on new facilities in the Unites States, and it is generally agreed that effective facilities planning can reduce material handling cost by at least 10 to $30 \%$. The size of the investment in new facilities each year makes the field of facilities planning important.

\subsection{Objectives of the Plant Layout Strategy}

Some typical plant layout objectives are to

- Meet the economic demand: minimize investment in equipment and material handling cost.

- Meet the requirement of product design and volume.

- Meet the requirement of process equipment and capacity: minimize overall production time; maintain flexibility of arrangement and operation; minimize variation in types of material handling equipment; facilitate the manufacturing process.

- Meet the requirement of quality of work life: provide for employee convenience, safety and comfort; facilitate the organizational structure.

- Meet the requirement of building and site constraints: utilize existing space most effectively. 


\subsection{Types of Layouts}

Layouts can be classified as seven types: fixed position layout; process oriented layout, also called job shop; group layout; office layout; retail/service layout; warehouse layout; product-oriented layout.

\subsection{Layout Design Methods and Computer Packages}

It is highly desirable that the optimum plant layout be designed. Unfortunately, the magnitude of the problem is so great that true system optimization is beyond current capabilities. The approach normally taken in solving the plant layout problem is to try to find a satisfactory solution. Previously, facilities layout problems were solved primarily by using iconic models. Then analytical approaches were developed.

In general, plant layout problems can be solved by any of the following approaches:

- Exact mathematical procedures.

- Heuristics.

- Probabilistic approaches.

- Graph theory.

A number of different procedures have been developed to aid the facilities planner in designing layouts. These procedures can be classified into two main categories: construction type and improvement type. Construction type layout methods basically 
involve developing a new layout from scratch. Improvement procedures generate layout alternatives based on an existing layout.

Based on the above two procedures, many algorithmic approaches have been developed. Some of them are Systematic Layout Planning (SLP) procedure, steepest descent search method by pairwise exchange, graph-based construction method, programming, network, Tabu search, simulated annealing and genetic algorithm. Based on these approaches, many computer-aided layout routines have been developed. Some of them are CRAFT [Buffa], COFAD [Tompkins], CORELAP [Lee], ALDEP [Seehof], PLANET [Tompkins], MULTIPLE [Bozer], BLOCPLAN [Katzel], SHAPE [Hassan], DISCON [Drezner] and SPS [Liggett].

There are also a few commercial packages available for facility layout design [Facilities Planning Software Buyer's Guide]. To name some, Archibus ${ }^{\circledR}$ is offered by Archibus Incorporation, Boston, Massachusetts. FactoryCAD $^{\circledR}$, FactoryPLAN ${ }^{\circledR}$ and FactoryFLOW $^{\circledR}$ are offered by Cimtechnologies Corporation, Ames, Iowa. LayOPT ${ }^{\circledR}$ is offered by Production Modeling Corporation, Dearborn, Michigan. FactoryModeler ${ }^{\circledR}$ is

offered by Systems Espace Temps, Inc., Cap Rouge, Quebec, Canada. PROMODEL ${ }^{\circledR}$ is offered by Promodel Corporation, Orem, Utah.

\subsection{Need for Research}

Hundreds of articles and dozens of computer programs have been written to solve facilities layout problems. Despite this, there are some key shortcomings of the existing programs. These drawbacks need further research. 


\subsubsection{Department shape and area}

A major drawback of existing layout procedures is how they represent a department within the boundary region. Layout problems are uniquely distinguished from other location problems by the existence of departmental areas. A number of existing algorithms represent departments as points, unit size, equal areas or circles [Drezner, 1980], rather than actual areas. Those procedures that consider the departments as areas often result in irregular shapes requiring considerable manual adjustment to create a useful layout.

\subsubsection{Flexibility}

Traditional approaches to the plant layout problem have assumed that the volume of flow between pairs of departments is deterministic. But it can easily be seen that production plans are subject to revisions due to changes in demand, product mix, new technology, etc. James A. Tompkins [Tompkins, 1996, p.307] noted some of these changes:

"Changes in the design of existing product, the elimination of products from the product line, and the introduction of new products; Changes in the processing sequences for existing products, replacements of existing processing equipment, and changes in the use of general-purpose and special purpose equipment; Changes in production quantities and associated production schedules, resulting in the need for capacity changes; Changes in the organizational structure as well as changes in management philosophies concerning 
production strategies such as the adoption of Just-in-Time concepts, Total Quality Management, etc."

Due to these changes the schedule for production will be subject to change, in which case the volume of flow among the departments would also change. Therefore, the layout should be designed to accommodate such changes. Flexibility in facilities design is often defined as the capability of a layout to react to disturbances caused by future events [Webster, 1980]. That means a layout that can still operate efficiently under a dynamic manufacturing environment. A flexible layout is desirable because it can handle future changes in product mix, product volume, the process, organizational structure and technology. If a layout is not flexible, under a variety of circumstances, the layout is not able to operate efficiently, so a rearrangement cannot be avoided. Besides rearrangement cost, this will raise some problems such as production shutdown during the rearrangement and resistance from management.

\subsubsection{Objectives}

The practical layout should meet multiple objectives, not a single objective. But most of the approaches consider only one objective. The results cannot be directly used without some manual adjustment. Multiple objectives for layout, especially qualitative objectives, need further research.

\subsection{Research Objectives}

The main research objective of this dissertation is to present a methodology that allows flexibility to be incorporated into the design of a facility. 
Under the main research objective the various functions are

\subsubsection{Develop a forecasting tool that can be used to forecast the flow among departments.}

This forecasting tool involves taking historical data and projecting them into the future with a statistical model. Some of the typical forecasting models are moving averages method, exponential smoothing method and linear regression method. The statistical model used here is the linear regression method. The results are the prediction of the future material flows among departments. This information will be used for creating a layout.

\subsubsection{Develop a new heuristic method that can be used to design a flexible layout with minimum material handling cost.}

A flexible layout created by heuristic method of this research can accommodate changes in future production demand, product mix, etc. Rearrangement is not needed so there is no rearrangement cost. Furthermore, this heuristic method can reduce material handling cost by shortening the distance among the departments.

\subsubsection{Develop an evaluation tool to select the most flexible layout.}

This evaluation tool is used to analyze and evaluate the layout alternatives. The change in material handling cost due to the change in material flow will be analyzed. The most flexible layout will be selected from the candidate set. 


\subsubsection{Integrate the layout design and production schedule by incorporating the schedule into layout design.}

Several possible material flows, instead of one single flow, will be considered simultaneously at the stage of layout design. For every layout alternative, each material flow is scheduled to reduce the distance among departments. For different material flows, there are different schedules. Then the layout alternatives will be evaluated based on these schedules. By incorporating the future schedule into layout design, different future situations can be handled. The layout created is more flexible.

\subsubsection{Develop a computer program to integrate all of the above functions for user to design a flexible layout.}

A computer program will be developed to implement and integrate all functions. The program will have a user-friendly interface and data management system, as well as algorithms. Users can use the program to design, evaluate and select a flexible layout.

\subsubsection{Analyze and evaluate several layout design methods including the method presented in this research by using case studies.}

Some case studies will be presented to compare this research's algorithm to some well-known computerized layout algorithms. Some algorithms and cases from the references will be selected for comparison.

\subsection{Conclusion}

The subject of layout design has been a popular topic for many years. It is one of the most popular subjects of current publications, conferences and research. From earlier discussions, it is evident that plant layout design plays a key role in the plant operation. 
A good layout well suited to the manufacturing philosophy is the fundamental starting point for total production system design. Because the volume and the mix of products to be produced is typically not known with certainty nor is it static over time, despite many existing layout design procedures the designed layouts don't have sufficient flexibility to meet the actual stochastic production requirements. Therefore, the approach of flexible layout design is necessary for manufacturing organizations to improve production operation and reduce operation cost. Flexible layout should give factories many practical advantages to remain competitive and on the first class level. 


\section{LITERATURE REVIEW}

\subsection{Layout Design Relationship}

The functions of product, process, schedule and layout design should interact. The facilities planner is dependent on timely and accurate input from product, process, and schedule designers to carry out his task effectively. Figure 2-1 [Tompkins, 1996] illustrates the need for close coordination among these four functions.

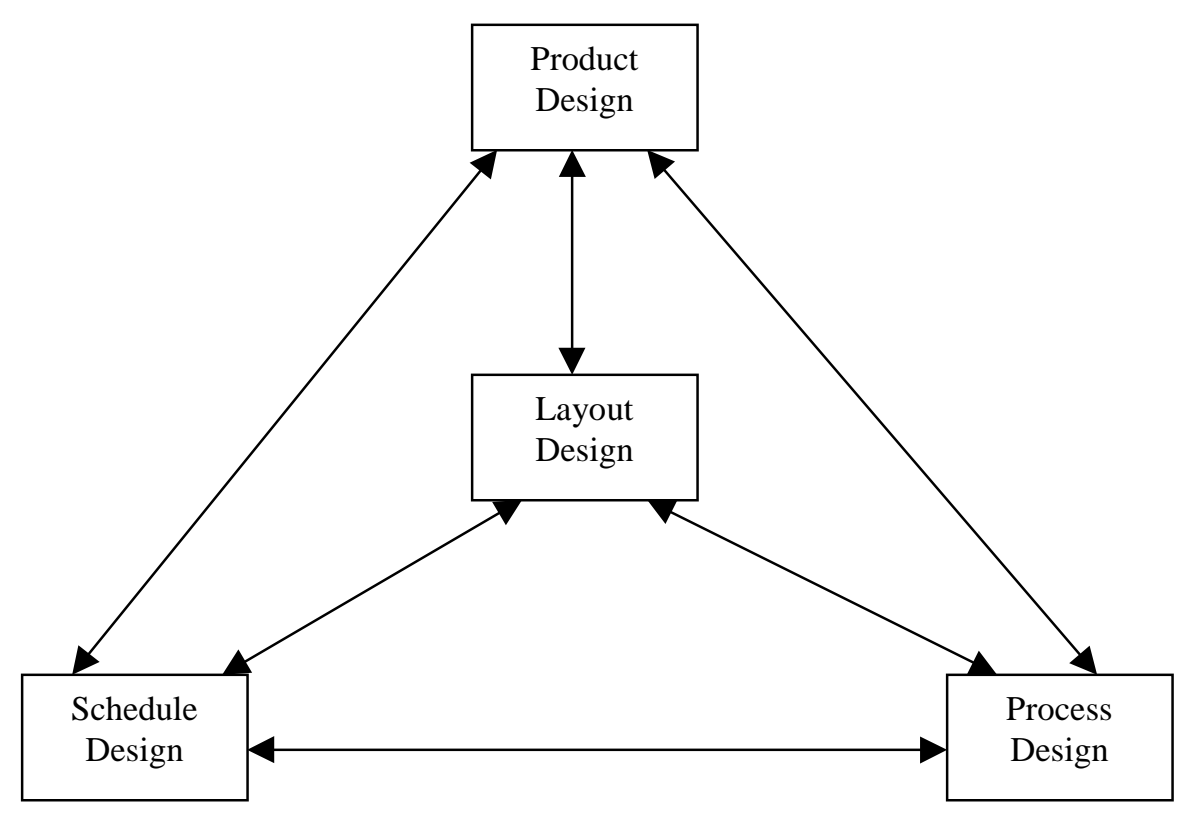

Figure 2-1 Relationship between Product, Process, Schedule and Layout Design

\subsection{Layout Design Procedures}

Among many different procedures, an organized approach to layout planning has been developed by Muther [Muther, 1973] and has received considerable publicity due to 
the success derived from its application in solving a large variety of layout problems. The approach is referred as Systematic Layout Planning (SLP). SLP has been applied to a variety of problems involving production, transportation, storage, supporting services and office activities.

The framework for SLP is given in Figure 2-2. The first five steps of SLP involve the analysis of the problem. Steps 6 through 9 constitute the search phase of the design process. The selection phase of the design process coincides with step 10.

\subsection{Layout Design Parameters}

Different procedures or computer-aided layout routines use different input data and control parameters, but some data are common: building dimensions, number of departments, shape and size/area of departments and material flow or relationship between departments.

\subsection{Layout Design Models}

Based on different algorithms, the layout problem can have different models. According to objective functions, there are two basic objectives [Tompkins, 1996]: one aims at minimizing the total material handling cost while the other aims at maximizing an adjacency score.

The formulation of the model for minimizing the total material handling cost is given as follows [Tompkins, 1996]: 


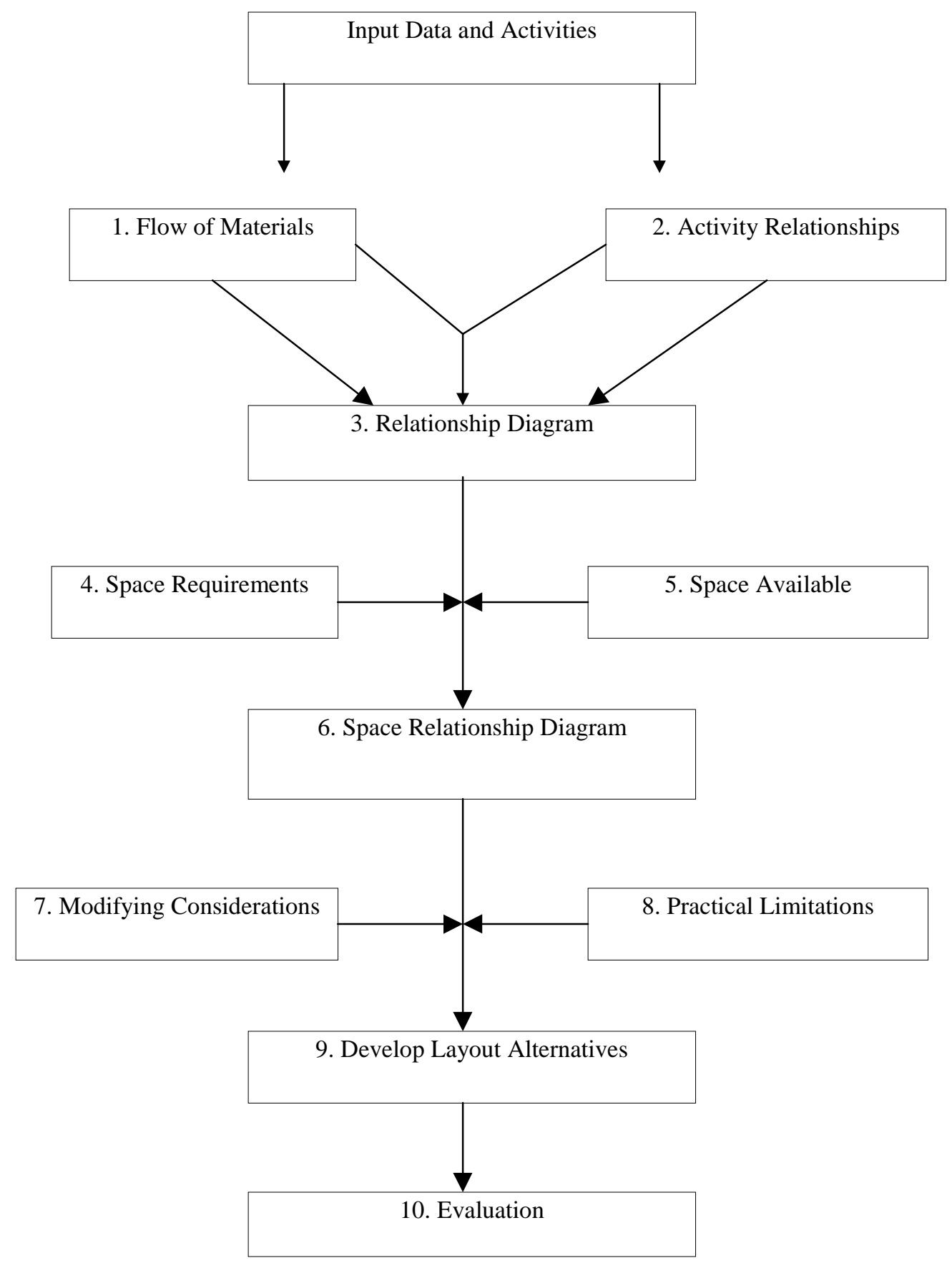

Figure 2-2 Systematic Layout Planning (SLP) Procedure [Muther, 1973] 
$\operatorname{Min} \mathbf{z}=\sum_{i=1}^{m} \sum_{j=1}^{m} \mathbf{f}_{\mathbf{i j}} \mathbf{c}_{\mathbf{i j}} \mathbf{d}_{\mathbf{i j}}$

Where

$$
\begin{aligned}
\mathrm{z} & =\text { total material handling cost. } \\
\mathrm{f}_{\mathrm{ij}} & =\text { material flow from department } \mathrm{i} \text { to department } \mathrm{j} . \\
\mathrm{c}_{\mathrm{ij}} & =\text { cost of moving a unit load one distance unit from department } \mathrm{i} \text { to } \\
& \text { department } \mathrm{j} . \\
\mathrm{d}_{\mathrm{ij}} & =\text { distance from department } \mathrm{i} \text { to } \mathrm{j} . \\
\mathrm{m} & =\text { number of departments. }
\end{aligned}
$$

The formulation of the model for maximizing an adjacency score is given as follows [Tompkins, 1996]:

$$
\operatorname{Max} \mathbf{z}=\sum_{i=1}^{m} \sum_{j=1}^{m} \mathbf{f}_{\mathbf{i j}} \mathbf{x}_{\mathbf{i j}}
$$

Where

$$
\begin{aligned}
\mathrm{Z} & =\text { adjacency score. } \\
\mathrm{f}_{\mathrm{ij}} & =\text { score between department } \mathrm{i} \text { and department } \mathrm{j} . \\
\mathrm{x}_{\mathrm{ij}} & =1, \text { if department } \mathrm{i} \text { and } \mathrm{j} \text { are adjacent in the layout. } \\
& =0, \text { otherwise. }
\end{aligned}
$$

\subsection{Computer-Aided Layout Design Software and Companies}

There are two basic types of programs among computer-aided facilities planning [Hales, 1984]. One is the improvement program. This kind of programs starts with a 
feasible solution, i.e. initial layout. They try to improve this initial layout to get a better solution. Usually quantitative flow inputs like from-to chart are used here. Examples of these programs are MULTIPLE [Bozer, 1994] and BLOCPLAN [Katzel, 1987]. Another one is the construction program. This kind of program generally develops a layout in an open area. Usually qualitative flow inputs like relationship chart are used here. Examples of these programs are SHAPE [Hassan, 1986] and SPS[Liggett, 1981].

\subsubsection{CRAFT}

CRAFT stands for Computerized Relative Allocation of Facilities Technique. It was introduced in 1963 by Buffa, Armour and Vollman [Buffa, 1963]. It is one of the earliest layout algorithms. It is a heuristic improvement routine. Its objective is to minimize transportation cost $=($ From-to matrix $) *($ Move cost matrix $) *($ Distance matrix). Its result is local optimal.

CRAFT has some assumptions: there are no "negative" relationships; all flows start and stop at department centroids; all movements are rectilinear; move costs are independent of equipment utilization; and move costs are linear with distance.

CRAFT needs the following input data: initial layout (building dimensions, number of departments, departments' areas, departments' position); from-to material flow matrix; move cost matrix; and number and location of fixed departments.

CRAFT has the following parameters to control the algorithm: same area interchange restriction to be relaxed to varying degrees; two or three-way interchanges; and significant level to make exchanges. 
CRAFT uses dummy departments for the following purposes: fixed to a specific area; make layout rectangular or square; represent fixed facilities such as stairways, elevators, restrooms and docks; represent aisles; and fill building.

CRAFT uses the following procedures to improve a layout:

- Place the layout on a coordinate system.

- Determine the centroids of the departments in initial layout.

- Calculate distance matrix between departments.

- Calculate the transportation cost by multiplying the distance matrix by the from-to matrix and by the move cost matrix.

- Make the interchange that offers the greatest estimated reduction in transportation costs. Check all possible interchanges that have equal areas or common borders.

- Repeat until no improvement is found.

CRAFT can have the following interchanges: pair-wise interchanges; three-way interchanges; pair-wise followed by three-way interchanges; three-way followed by pair-wise interchanges; the best of pair-wise or three-way interchanges.

CRAFT can do the sensitivity analysis by using various initial layouts or by using various from-to values.

CRAFT has the following limitations: 
- It cannot handle a change in material flow. It assumes the material flow is deterministic. For different material flows it creates different layouts. Each layout is only used for a specific situation.

- Number of departments is less than 40 .

- Path dependence: Different initial layouts give different final solutions.

- Department shapes deteriorate rapidly with the number of iterations. Outputs contain unrealistic locations, shapes, and alignments. Manual adjustments are always required.

- The improvement algorithms cannot generally consider a negative "X" relationship.

- The improvement algorithms do not deal easily with other-than flow relationship.

- Architectural influences and other qualitative factors are very difficult to consider. They are usually ignored.

- Costs may not be significant, known, and linear in distance as assumption. Maybe there is more than one piece of material handling equipment between two departments. In this case, there is more than one material handling cost between two departments. For example, CRAFT has only one material handling cost $c_{i j}$ from department $i$ to department $j$. The user cannot define more than one $c_{i j}$ and cannot have different flows from department $i$ to department $\mathrm{j}$.

Examples of CRAFT are presented in Chapter 5. 


\subsubsection{COFAD}

COFAD stands for COmputerized FAcilities Design. It was introduced by Tompkins. It is also an improvement routine. It is an improved version of CRAFT [Tompkins, 1984]. Its objective is also to minimize material-handling cost. But it tries to consider layout and material handling systems simultaneously.

COFAD needs the following input data: initial layout; from-to matrix; and move cost equations. The move cost equations depend on the material handling equipment:

- Fixed path equipment ( conveyors, cranes, hoists, etc. )

FPMC $=$ FPFC + FPVC $*$ ML

where

$\begin{array}{lll}\text { FPMC } & =\text { Fixed Path Move Cost } & (\$ / \mathrm{yr}) \\ \text { FPFC } & =\text { Fixed Path Fixed Cost } & (\$ / \mathrm{yr}) \\ \text { FPVC } & =\text { Fixed Path Variable Cost } & (\$ / \mathrm{ft}) \\ \text { ML } & =\text { Move Length } & (\mathrm{ft} / \mathrm{yr})\end{array}$

- Variable path equipment ( Vehicles: truck, platform truck, etc. )

VPMC $=$ VPFC $*$ EUT + VPVC $*$ MTIME

where

$\begin{array}{llr}\text { VPMC } & =\text { Variable Path Move Cost } & (\$ / \mathrm{yr}) \\ \text { VPFC } & =\text { Variable Path Fixed Cost } & (\$ / \mathrm{yr}) \\ \text { EUT } & & =\text { Equipment UTilization for this move }(\%) \\ \text { VPVC } & =\text { Variable Path Variable Cost } & (\$ / \mathrm{hr})\end{array}$




$\begin{array}{rlr} & =\text { ANTC/OPHY } \\ \text { ANTC } \quad= & \text { ANnual Total variable Cost } & (\$ / \mathrm{yr}) \\ & (\text { labor power, maintenance }) & \\ \text { OPHY } \quad & \text { OPerating Hours per Year } & (\mathrm{hr} / \mathrm{yr}) \\ \text { MTIME } \quad= & \text { Move TIME } & (\mathrm{hr} / \mathrm{yr}) \\ & & \\ & & \end{array}$

COFAD uses the following procedures to improve a layout:

- Improve initial layout ( CRAFT-like procedure).

- Determine costs of all feasible material handling equipment. Select the one with the lowest cost.

- Calculate the utilization of each type of equipment. Calculate the deviation (differences) for each type of equipment.

- Transfer some assignments from the equipment type with the largest deviation to the equipment type with the smallest deviation to minimize the total deviations.

- For each move the cost of which is greater than original move cost, try all feasible equipment types and recalculate the total cost to check whether reassign it or not to get a minimal cost material handling system.

- Repeat until change in cost and number of changes in material handling system assignments is less than the initially input steady-state percentage.

COFAD can do sensitivity analysis by varying the flow volume or by changing the product mix. 
Because COFAD is similar to CRAFT, it has the same limitations as CRAFT.

\subsubsection{CORELAP}

CORELAP stands for COmputerized RElationship LAyout Planning. It was developed by Lee and Moore in 1967 [Lee, 1967]. CORELAP is the oldest and best known construction routine. Its objective is to create a layout with "high-ranking" departments close together. It is a computerized version of Muther's Systematic Layout Planning(SLP) [Muther, 1973, 1961]. The approximations used in the relationship diagram may be more appropriate than the exact cost approach of CRAFT and COFAD because of lack of data.

The assumption of CORELAP is that the department will have a dispatch area and a receiving area on the side of its layout nearest its neighbor.

The input data of CORELAP are number of departments; department areas; relationship chart; and weights for relationship chart. The optional input data are scale of output printout; length to width ratio; and department pre-assignment (only along the periphery of the layout ).

CORELAP uses the letter symbols A, E, I, O, U and $\mathrm{X}$ for the closeness relationship:
$\mathrm{A}=$ Absolutely necessary
$\mathrm{E}=$ Especially important
$\mathrm{I}=$ Important 


$$
\begin{array}{lll}
\mathrm{O} & = & \text { Ordinary closeness OK } \\
\mathrm{U} & = & \text { Unimportant } \\
\mathrm{X}= & \text { Not desirable }
\end{array}
$$

The letter ratings are converted to their numerical equivalents $(\mathrm{A}=6, \mathrm{E}=5$, etc. $)$. The weighted relationship values ( $\mathrm{A}=3^{5}=243, \mathrm{E}=3^{4}=81$, etc. $)$ are used for placing departments.

Selection procedure (sequence) of CORELAP:

- Calculate the Total Closeness Rating (TCR):

$$
\mathrm{TCR}_{\mathrm{i}}=\sum_{\mathrm{j}=1}^{\mathrm{m}} \mathrm{V}_{\mathrm{ij}}
$$

where $\mathrm{V}_{\mathrm{ij}}=$ Values of relationships between department $\mathrm{i}$ and

$$
\text { all other departments. }\left(\mathrm{V}_{\mathrm{ii}}=0\right)
$$

- Select the department with the highest TCR as the first one.

Tie-breaking rule:

. department having the largest area

. department having the lowest department number

- Scan the relationship chart. Select the department having A with the first one. If none, then E. ... If two or more, select the one having the highest TCR. If still tied, use tie rule.

- For the third one, select the department having A with the first one. If none, select the department having A with the second one. If none, select the department having $\mathrm{E}$ with the first one. ...

- Repeat until all the departments are selected. 
Placement decision of CORELAP:

- Locate rectangular shape departments if permitted. Length-to-width is determined by user.

- Place the first department at the center of layout.

- Calculate the Placing Rating (PR) for all available locations. $\mathrm{PR}=\Sigma$ (weighted closeness ratings between the department to be placed and its neighbors already in layout).

- Choose the location with the highest placing rating.

- Tie-breaking: Take the one with the largest boundary length. Boundary length $=$ (number of unit square sides that the department to be placed has in common with its neighbors).

Evaluation of layout created by CORELAP:

- Calculate the layout score:

Layout score $=\Sigma_{\text {all dept. }}($ Closeness rating $) *($ Length of shortest path $)$

- The lower, the better.

- Path is rectilinear between departments, not between centroids as in CRAFT, COFAD, and PLANET.

Sensitivity analysis by CORELAP:

- Change the relationship chart.

- Change the weighted rating values.

- Change the departmental areas.

- Change the layout scale (unit square). 
- Change the value of the length-to-width ratio.

Limitations of CORELAP:

- It cannot handle a change in relationship among departments. If a relationship changes, CORELAP has to create a new layout.

- The building shape may be irregular. Manual adjustment is needed. An example of a layout created is like this:

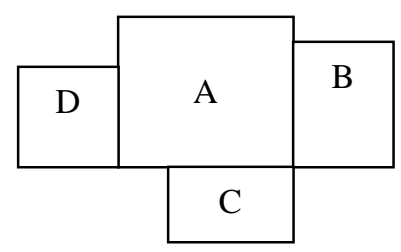

- Shortest rectilinear path may not always be a realistic measure.

Limitations for general construction routines:

- Ignores the direction of flow among departments.

- Some important relationships are not considered.

- It is the departments instead of relationships that are considered in order of priority or importance.

An example of CORELAP is presented in Chapter 5.

\subsubsection{ALDEP}

ALDEP stands for Automated Layout DEsign Program. It was developed by Seehof and Evans [Seehof, 1967]. It is also a construction routine. ALDEP is a variation of CORELAP. Its objective is also to create a layout with "high-ranking" departments 
close together. But ALDEP has special characteristics of randomness, up to three floors capability and departments ( docks, elevators, aisles ) that can be fixed.

The input data of ALDEP are length, width, and area of each floor; location and size of restricted area for each floor; scale of layout printout; number of layouts to be generated; number of departments; department areas; relationship chart; and minimum allowable score for an acceptable layout.

Selection procedure (sequence) of ALDEP:

- Randomly select a department.

- Add a department with an important relationship with previous departments. If none, add an unimportant department randomly.

- Continue until all departments are added.

Placement decision of ALDEP:

- All departments are square or rectangular

- First department is put at the upper left corner

- Use "sweep" method to locate next department. Figure 2-3 shows an example of this method.

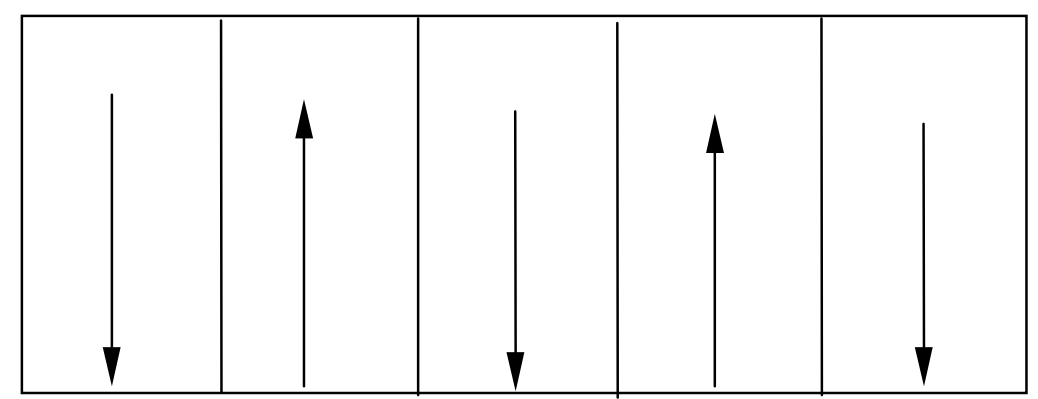

Figure 2-3 "Sweep" Method for ALDEP 
Evaluation of layout created by ALDEP:

- Check all adjacent departments. Sum the closeness values as rating value of this layout.

\section{Limitations of ALDEP:}

- It cannot handle a change in relationship among departments. If a relationship changes, ALDEP has to create new layouts.

- It ignores the direction of flow among departments.

- Some important relationships will not be considered.

- It is the departments instead of relationships that are considered in order of priority or importance.

Differences between ALDEP and CORELAP:

- By procedure: ALDEP selects the first department randomly. CORELAP selects the first department according to Total Closeness Rating.

- By philosophy: ALDEP generates many layouts and rates each layout (up to 20). CORELAP generates one best layout.

An example of ALDEP is presented in Chapter 5.

\subsubsection{PLANET}

PLANET stands for Plant Layout ANalysis Evaluation Technique. It was developed by Deisenroth and Apple [Konz, 1985]. It is also a construction routine. But its 
objective is to minimize the material handling cost $=($ From-to matrix $) *($ Distance moved matrix).

Assumptions of PLANET are all moves are rectangular from the department centroid; and move cost is linear with move length and independent of equipment utilization.

\section{Input data of PLANET:}

- Number of departments and areas.

- Priority of the placement of department into the layout (Highest 1 --- 9 lowest).

- Material flow data (one of three methods): from-to chart; penalty matrix (Penalty value $=-9$--- 99. The larger the penalty value, the more important the closeness); production data (Production sequence for each part; flow volumes; and cost/move/100ft for each part [ It can be simplified to unity]).

- Change material flow data to flow-between cost chart.

Selection procedure (sequence) of PLANET:

- Method A:

- The first pair of departments to enter must be in the highest priority group and have the highest flow-between cost.

- The next one will have the highest flow-between cost with any department in the layout and is in the highest priority group.

- Continue until all departments are selected.

- Method B:

- The first pair is like method A. 
- The next one is in the highest priority group and has the highest sum of flow-between costs with all departments in layout.

- Continue until all departments are selected.

- Method C:

- The first department is in the highest priority group and has the highest sum of flow-between costs with all other departments.

- Continue until all departments are selected.

Placement decision of PLANET:

- The first two are adjacent in the center.

- Locate the next one in order to minimize the increase in handling cost. Centroids and distances between centroids and each point around the perimeter of the existing layout are first determined. Volume-distance $=($ distance $)($ flow-between cost chart).

- Continue until done.

Limitations of PLANET:

- It cannot handle a change in material flow. It uses deterministic material flow to create the layout.

- It ignores the direction of flow among departments.

- It cannot fix departments.

- It may generate unrealistic shapes.

- It cannot be used to evaluate the effects of manually adjusting the layout. 


\subsubsection{MULTIPLE}

MULTIPLE stands for MULTI-floor Plant Layout Evaluation. It was developed by Bozer, Meller and Erlebacher [Bozer, 1994]. It is an improvement algorithm. It is similar to CRAFT, but it can exchange any two departments despite their areas. It can control department shapes and use flexible department areas $\left(\mathrm{A}_{i}^{\mathrm{L}} \leq \operatorname{Area}_{i} \leq \mathrm{A}_{\mathrm{i}}^{\mathrm{U}}\right)$.

The objective of MULTIPLE is to minimize

$$
\text { Moving cost }=\quad \Sigma_{i=1}{ }^{N} \Sigma_{j=1}{ }^{N}\left(C_{i j}{ }^{H} d_{i j}^{H}+C_{i j}{ }^{V} d_{i j}{ }^{V}\right) f_{i j}
$$

where

$$
\begin{aligned}
\mathrm{C}_{\mathrm{ij}}{ }^{\mathrm{H}} & =\text { Horizontal cost / unit load / unit distance } \\
\mathrm{C}_{\mathrm{ij}}{ }^{\mathrm{V}} & =\text { Vertical cost / unit load / unit distance } \\
\mathrm{d}_{\mathrm{ij}}{ }^{\mathrm{H}} & =\text { Horizontal distance } \\
\mathrm{d}_{\mathrm{ij}}{ }^{\mathrm{V}} & =\text { Vertical distance } \\
\mathrm{f}_{\mathrm{ij}} & =\text { Flow from department } \mathrm{i} \text { to } \mathrm{j}
\end{aligned}
$$

MULTIPLE uses a different layout representation from CRAFT. It uses a matrix to represent the layout. Each element represents a grid. The area of a department is represented by a certain number of grids. The spacefilling curve is used to visit all the grids except fixed departments.

MULTIPLE's exchange with unequal areas:

- Exchange department $i, j$. Say $A_{i}>A_{j}$.

- If $\mathrm{A}_{\mathrm{i}}^{\mathrm{L}} \leq \mathrm{A}_{\mathrm{j}}, \mathrm{A}_{\mathrm{j}}^{\mathrm{U}} \geq \mathrm{A}_{\mathrm{i}}$, make the exchange by compressing department $\mathrm{i}$ and expanding department $\mathrm{j}$. 
- If not, make the exchange by compressing the departments around $\mathrm{j}$ and expanding the departments around i.

- Make the exchange without compressing or expanding. Move all departments between department $\mathrm{i}$ and $\mathrm{j}$ along the spacefilling curve.

MULTIPLE's measure of department shape:

- Shape irregularity $=$ Perimeter $/$ Area $=P_{i} / A_{i}$

- Square shape: $\mathrm{P}_{\mathrm{i}}^{*}=4 \sqrt{\mathrm{A}_{\mathrm{i}}}$

- Shape measure: $\Omega_{\mathrm{I}}=\left(\mathrm{P}_{\mathrm{i}} / \mathrm{A}_{\mathrm{i}}\right) /\left(\mathrm{P}_{\mathrm{i}}^{*} / \mathrm{A}_{\mathrm{i}}\right)=\mathrm{P}_{\mathrm{i}} \mathrm{A}_{\mathrm{i}}^{-0.5} / 4$

Algorithm of MULTIPLE:

- Consider all two-way, area-feasible exchanges.

- Calculate the costs.

- Check other constraints for the best exchange with minimum cost.

- Take this best exchange if feasible.

- Repeat until no improvement.

Because the overall approach of MULTIPLE is similar to CRAFT, its limitations are also similar to CRAFT.

\subsubsection{BLOCPLAN}

BLOPLAN stands for Block Layout Overview with Computerized Planning using Logic and Algorithms: New issue. It was developed by Donaghey and Pire [Katzel, 
1987]. It is a menu-driven, conversational routine. It is used for a layout that has fewer than 18 departments.

Input data of BLOCPLAN: number of departments; size and area of each department; relationship chart; fixed departments; and seven choices (three algorithms, adjust relationship, manually insert departments, review saved layouts, stop).

Three logic algorithms:

- Improvement: uses initial layout and pair exchange.

- Construction: puts highest rating department to center. There is some randomness.

- Random: creates a variety of random layouts and gives the score compared to the best theoretical layout.

\section{Limitations of BLOCPLAN:}

- It cannot handle a change in material flow. It uses fixed flows to create the layout.

- Maximum number of departments is 18 .

- The unit costs must be symmetric between two departments. That means $\mathrm{c}_{\mathrm{ij}}=\mathrm{c}_{\mathrm{ji}}$.

\subsubsection{SHAPE}

SHAPE stands for Selection of materials Handling equipment and Area Placement Evaluation. It was developed by Hassan, Hogg and Smith [Hassan, 1986]. It is a 
heuristic construction algorithm. Its objective is to minimize total movement costs $=$ (move distance) (material volume).

Formulation of SHAPE:

- Represents the layout by a matrix of a unit square.

- User selects the size of unit squares. The size of unit affects the overall layout solution.

- The departments enter the layout like the new items enter the warehouse.

- Symbols:

$\mathrm{N} \quad=$ dimension of layout region, unit squares.

$\mathrm{A}_{\mathrm{i}} \quad=$ area of department $\mathrm{i}$, unit squares.

$\mathrm{n} \quad=$ number of departments.

$\mathrm{d}_{\mathrm{ij}} \quad=$ distance between center of department $\mathrm{i}$ and center of unit square $\mathrm{j}$.

$\mathrm{f}_{\mathrm{ik}} \quad=$ flow between departments $\mathrm{i}$ and $\mathrm{k}$.

$\sum_{\mathrm{j}}^{\mathrm{Ak}} \mathrm{d}_{\mathrm{ij}} / \mathrm{A}_{\mathrm{k}}=$ average distance that $\mathrm{f}_{\mathrm{ik}}$ travels.

$\mathrm{x}_{\mathrm{kj}} \quad=1$ if $\mathrm{j} \in \mathrm{k}$.

$=0$ if $\mathrm{j} \notin \mathrm{k}$.

- Model:

$\operatorname{Min} \sum_{k=2}{ }^{n} \sum_{i=1}^{k-1} f_{i k} \sum_{j=1}{ }^{N}\left(d_{i j} / A_{k}\right) x_{k j}$

Subject to

$$
\begin{array}{lll}
\sum_{\mathrm{j}=1}{ }^{\mathrm{N}} \mathrm{x}_{\mathrm{kj}} & =\mathrm{A}_{\mathrm{k}}, & \mathrm{k}=1, \ldots, \mathrm{n} \\
\sum_{\mathrm{k}=1}^{\mathrm{n}} \mathrm{x}_{\mathrm{kj}} & =1, & \mathrm{j}=1, \ldots, \mathrm{N}
\end{array}
$$




$$
\mathrm{x}_{\mathrm{kj}} \quad=\quad\{0,1\} \quad \forall \mathrm{j}, \mathrm{k}
$$

Selection procedure (sequence) of SHAPE:

- Similar to PLANET \& CORELAP.

- From-to chart is used as relationship chart.

- Classify flow-value as major or minor.

- $\quad$ Rank $=\sum$ major flow values.

Placement and construction of SHAPE:

- The departments are square or rectangular.

- The first one is placed in the center.

- The next one is around the previous departments. Check the four sides of entered layout. Take the side with the minimum movement cost.

- Departments with lesser flow rates are put on corners or sides.

\section{Limitations of SHAPE:}

- It cannot handle a change in material flow. Deterministic flow is used to create the layout.

- Some important relationships will not be considered.

- It is the departments instead of relationships that are considered in order of priority or importance.

- The building shape may be irregular. Manual adjustment is needed. 


\subsubsection{DISCON}

DISCON stands for DISpersion-CONcentration. It was developed by Drezner [Drezner, 1980]. It has some special assumptions: departments have circular shapes; and the distance is from center to center.

DISCON uses Lagrangian Differential Gradient (LDG) method. Formulation of DISCON:

$$
\begin{aligned}
\operatorname{Min} \mathbf{F}(\mathbf{x}, \mathbf{y}) & =\quad \sum_{i=1}{ }^{n-1} \sum_{j=i+1}{ }^{n} C_{i j} d_{i j} \\
& =\quad \sum_{i=1}{ }^{n-1} \sum_{j=i+1}{ }^{n} C_{i j}\left[\left(x_{i}-x_{j}\right)^{2}+\left(y_{i}-y_{j}\right)^{2}\right]^{1 / 2}
\end{aligned}
$$

Subject to

$$
\lambda\left(\mathrm{R}_{\mathrm{i}}+\mathrm{R}_{\mathrm{j}}-\mathrm{d}_{\mathrm{ij}}\right) \quad \leq \quad 0, \quad \lambda>0
$$

Where

$$
\begin{array}{ll}
\mathrm{n} & =\text { number of departments } \\
\mathrm{d}_{\mathrm{ij}} & =\text { distance between centers of departments } \mathrm{i} \text { and } \mathrm{j} \\
\mathrm{x}_{\mathrm{i}}, \mathrm{y}_{\mathrm{i}} & =\text { coordinate value of center of department } \mathrm{i} \\
\mathrm{C}_{\mathrm{ij}} & =\text { cost/unit distance between } \mathrm{i} \text { and } \mathrm{j} \\
\mathrm{R}_{\mathrm{i}} & =\text { radius of department } \mathrm{i}
\end{array}
$$

The procedure of DISCON is that first there is a dispersion. All departments are in the same center and explode to get the initial layout. Then there is a concentration. The departments are compressed to a dense arrangement to get the local optimal.

\section{Limitations of DISCON:}


- It cannot handle a change in material handling cost. The fixed cost/unit distance [i.e., (flow)x(cost/unit flow/unit distance)] is used to create a layout.

- The department shape is circular, which is not normal.

- Use of centroid locations in measuring distances is sometimes not a good method.

\subsubsection{Space Planning System}

The Space Planning System (SPS) was developed by Liggett and Mitchell [Liggett, 1981]. It combines the construction and improvement algorithms. First it uses sophisticated constructive initial placement strategy, then uses simple iterative improvement strategy. It also uses probability theory and gives sensitivity information.

The formulation of SPS:

Minimize $\quad \rho \in \mathrm{S} \Sigma_{\mathrm{i}} \mathbf{f}_{\mathrm{i} \rho(\mathrm{i})}+\Sigma_{\mathrm{i}} \Sigma_{\mathrm{j}} \mathbf{q}_{\mathrm{ij}} \mathbf{c}_{\rho(\mathrm{i}) \rho(\mathrm{j})}$

Where:

$$
\begin{array}{ll}
\mathrm{M} & =\{1, \ldots, \mathrm{m}\} \quad \text { ( set of grids of all departments ) } \\
\mathrm{N} & =\{1, \ldots, \mathrm{n}\} \text { ( set of grids of plane ) } \\
\mathrm{S} & =\text { set of all possible mappings: } \mathrm{M} \rightarrow \mathrm{N} \\
\rho & =\text { a particular map, } \rho \in \mathrm{S}, \rho(\mathrm{i} \in \mathrm{M})=\mathrm{j} \in \mathrm{N} \\
\mathrm{f}_{\mathrm{ij}} & =\text { fixed cost of assigning } \mathrm{i} \in \mathrm{M} \rightarrow \mathrm{j} \in \mathrm{N} \\
\mathrm{q}_{\mathrm{ij}} \quad \quad \text { interaction between } \mathrm{i} \text { and } \mathrm{j} \in \mathrm{M} \\
\mathrm{c}_{\mathrm{ij}} \quad=\text { distance between } \mathrm{i} \text { and } \mathrm{j} \in \mathrm{N}
\end{array}
$$

The constructive placement stage of SPS: 
- Pre-assign fixed departments.

- All unassigned department-location combinations are evaluated at each step.

- Use probability theory to predict the effect of a particular assignment ( Expected value of the objective ).

- Sort departments by shape ratio. Proportion shape ratio $=$ (short side of bounding rectangle) / (long side). Coherence shape ratio $=$ (number of units in department) / (number of units in bounding rectangle).

- $\quad$ Place first group (size P), then next group, ...

The improvement stage of SPS:

- Simple pair-wise exchange.

- Modify shape according to shape ratio.

The limitations of SPS:

- It cannot handle a change in material flow. Deterministic flow is used to create a layout.

- The building shape may be irregular. Manual adjustment is needed.

- Few departments have exactly the same area.

\subsection{Current Flexible Layout Design Research}

Flexibility in facilities design is often defined as the capability of a layout to react to disturbances caused by future events [Webster, 1980]. Some typical changes are in product mix, product volume, the process, organizational structure and technology. 
Some work has been done in flexible layout design. Tompkins [Tompkins, 1996] and Langevin [Langevin, 1994] used the spine method to handle flexibility. It requires all material flow in the plant to be channeled through a 'spine' that remains unchanged with variations in product volume and mix. Sometimes the departments need to be rearranged although it is somewhat easy in this situation. The approach makes the material handling flexible, but it does not reduce the distance among departments when there is a change in the material flow.

Lacksonen [Lacksonen, 1994], Balakrishnan [Balakrishnan, 1993], Afentakis [Afentakis, 1990], and Rosenblatt [Rosenblatt, 1986] developed dynamic layouts to handle flexibility. The general dynamic layout problem is to find a series of block layouts that minimize the flow costs at each time period and the rearrangement costs between time periods. The main characteristic here is that rearrangement is needed. Besides rearrangement cost, this will raise some problems such as production shutdown during the rearrangement and resistance from management. Montreuil [Montreuil, 1991] developed dynamic layouts using a different philosophy. He didn't design one layout for each time period but only designed one final layout. Then a linear programming model was used to conduct the previous phase layouts that should 'grow up' to a final layout. The main advantage here is that there is no rearrangement, but how to shorten the distance among departments and how to utilize the reserved area for expansion are not considered.

Dahel [Dahel, 1993] utilized Group Technology (GT) to design a flexible layout. Group Technology is another relatively new manufacturing philosophy that can address 
the problem of flexibility. This requires that the machines that need to produce a family of parts can be grouped into a machine cell. GT tries to absorb the effects of changing product volume and mix within the cell, but when there is a change in material flow, inter-cell flow usually cannot be avoided. That will increase the material handling cost and affect the cell utilization.

Bozer [Bozer, 1997] challenged the assumption that the distance is from centroid to centroid. The expected-distance was used in his paper for material handling cost calculation. His objective was to make measurement of distance more reasonable, but his research was still based on deterministic situation. Flexibility was not considered in his paper. Bozer [Bozer, 1994] also developed an algorithm called MULTIPLE which is similar to CRAFT, but it considered multiple floors, and it could exchange two departments more easily and accurately by using spacefilling curves. However, flexibility was not considered. Jajodia [Jajodia, 1992] developed an algorithm called CLASS. A simulated annealing method was used in CLASS. The simulated annealing method can relax the pair-exchange method's 'path dependent' limitation and improve the result, but flexibility is not considered.

Heragu [Heragu, 1991] developed both linear continuous and linear mixed integer models for layout design. Montreuil [Montreuil, 1993] developed a linear programming model for layout design. They tried to get optimal solutions for small-size problems, but none of these models involved flexibility. Meller [Meller, 1996] combined the distance objective and adjacent objective to get a weighted objective. Then he designed a layout in terms of this weighted objective. A layout that is insensitive to different weights is 
called a robust layout. Rosenblatt [Rosenblatt, 1992] used expected flow to create a flexible layout. A comparison of Rosenblatt's result with the results from the algorithm presented in this research is given in Chapter 5. Sly [Sly, 1995] described an interactive 3-D animated graphics method integrated with MRP systems, spreadsheets, databases and simulation systems. It is based on AutoCAD. It focuses mainly on interface and graphics, not optimization, and it doesn't consider flexibility.

Some approaches used the criteria to select the flexible layouts. Tompkins [Tompkins, 1980] used penalty to quantify/measure flexibility. These kinds of approaches did not have algorithms to create the layouts. They used other methods to create the layout alternatives. Then some criteria were used to select the flexible layouts. In these cases, the results depended on the layout candidate set created by other methods.

\subsection{Conclusion}

From the discussion above, it can be concluded that there have been numerous research activities in the areas of layout design. There have also been a number of algorithms for flexible layout design. However, it must be realized that the layout design problem is still under research. Therefore, the study reported here attempts to present a new method for flexible layout design and provide an analysis tool to evaluate the flexible layout alternatives. Although a lot of effort has been put into layout design, it seems that more research is needed to design better layouts to improve material handling efficiency. 


\section{RESEARCH APPROACH AND ALGORITHMS}

\subsection{Introduction}

In this section of the report, the overall development of a new layout design algorithm and computer-based system are outlined. Then the major algorithms are described in detail. A different approach is described for incorporating flexibility in layout designs. In brief, the approach for plant layout design comprises a flow forecasting tool, a pair exchange layout design algorithm, a new layout design algorithm, a scheduling tool, a layout drawing tool and an evaluation tool that gives the layout alternatives, analysis and evaluation.

Most of the factory layout software developed over the last 30 years has been grounded within the function factory paradigm [Sly, 1996]. In a function factory, processors having the same functional capabilities are organized into a function department. Function layout, also called job shop layout, has the advantage of handling flexibility but at the expense of material handling among the departments.

After the introduction of group technology, the cellular layouts are used where volume warrants a special arrangement of machinery and equipment. In a group factory, cells are formed around manufacturing responsibility for particular product families. All processors necessary for producing the entire demand for the product family are organized into a group cell. 
The traditional approach to cell formation is to create an independent cell for each part family. Machine sharing and intercell flows are discouraged. However, with product mixes and volumes being subject to change, traditional cells will affect machine utilization. Furthermore, intercell flows will occur in the future. So the cellular layout reduces the cost of material handling but can't easily handle flexibility.

The approach given here utilizes the advantages of both job shop layout and cellular layout. A hybrid layout is designed to integrate job shop layout and cellular layout to have the ability to handle flexibility.

\subsection{Assumptions in the Research}

The following assumptions were made for the method described in this research:

1. The factory is a process type factory.

2. All movement is between department centers.

3. The objective to be minimized is the material handling costs.

According to [Lacksonen, 1994], indirect costs are the largest portion of a company's total operating cost. To keep a company competitive, engineers focus on reducing the company's indirect costs. A major component of the indirect costs is material handling equipment and operation.

4. The travel distances are rectilinear or Euclidean.

Assumptions here are commonly encountered in the literature. 


\subsection{Flexible Layout Design System}

The flexible layout design algorithm given in this research is to locate the departments adjacent to each other. This adjacency of departments allows the change of material flow without physically relocating the departments. This will save rearrangement costs and avoid other rearrangement problem like rearrangement time and resistance from management.

It is not easy to make one department adjacent to all others. A different approach that is called flexible layout design algorithm is used to split the department into two or more portions as necessary. These portions will be placed into the different locations of the layout so that adjacency of departments can be satisfied. It is as though the layout consists of several cells, but they are not real cells; they are more like several subordinate job shops put together.

When material flows change due to a change of volume of products, product mix, etc., each flow between two departments needs to be rescheduled. It is like cells are reformed for this particular flow. So each time the flows change, the formation of cells is different without rearrangement. These cells can overlap without causing problems. Here when the layout is designed, the schedule is also considered and integrated into the layout design.

The layout created with more than one same department has two advantages. First, it reduces the distances among the departments. Hence, it reduces the total material handling cost. Therefore, it is efficient. Secondly, it provides the opportunity to reschedule future material flows in spite of their uncertainty at the design stage. It is the 
material flow that accommodates the layout, not the layout that accommodates the material flow. By changing the philosophy, we have a layout that is flexible for future changes. The pair exchange layout design algorithm is used to improve the layout. This algorithm and flexible layout design algorithm are working together to create a flexible layout.

For any layout designs which consider both known and future product mixes and volumes, the alternative with the lowest total material handling cost is considered to be the most flexible and hence the best layout. Minimum expected material handling cost is the criterion for determining the most flexible layout from among various alternatives. So the total expected material handling cost for a particular layout design is used to provide a quantitative measure of a design's flexibility.

Due to the uncertainty of future material flow, flow forecasting is also one portion of this research. Flow forecasting utilizes the linear regression method to create the forecasting results. This forecasting result will be used for layout design.

When the future material flow is finally known, the flows between the departments can be rescheduled to reduce the distances between the departments. So the layout is also flexible to any change of material flow that is different from any of forecasting material flows.

After the layouts are created, an evaluation method is presented to analyze and evaluate the layout alternatives created from the above flexible layout design algorithm and other methods and select the most flexible layout from the candidate set. The 
evaluation method utilizes probability theory. It analyzes the change in material handling cost due to the change in material flow and selects the one layout that is the most insensitive to the change in material flow as the most flexible layout.

\subsection{Computer Program Data Flow Diagram}

A computer program was developed to implement and integrate the above methods for users to use for layout design. The program flow diagram is shown in Figure 3-1.
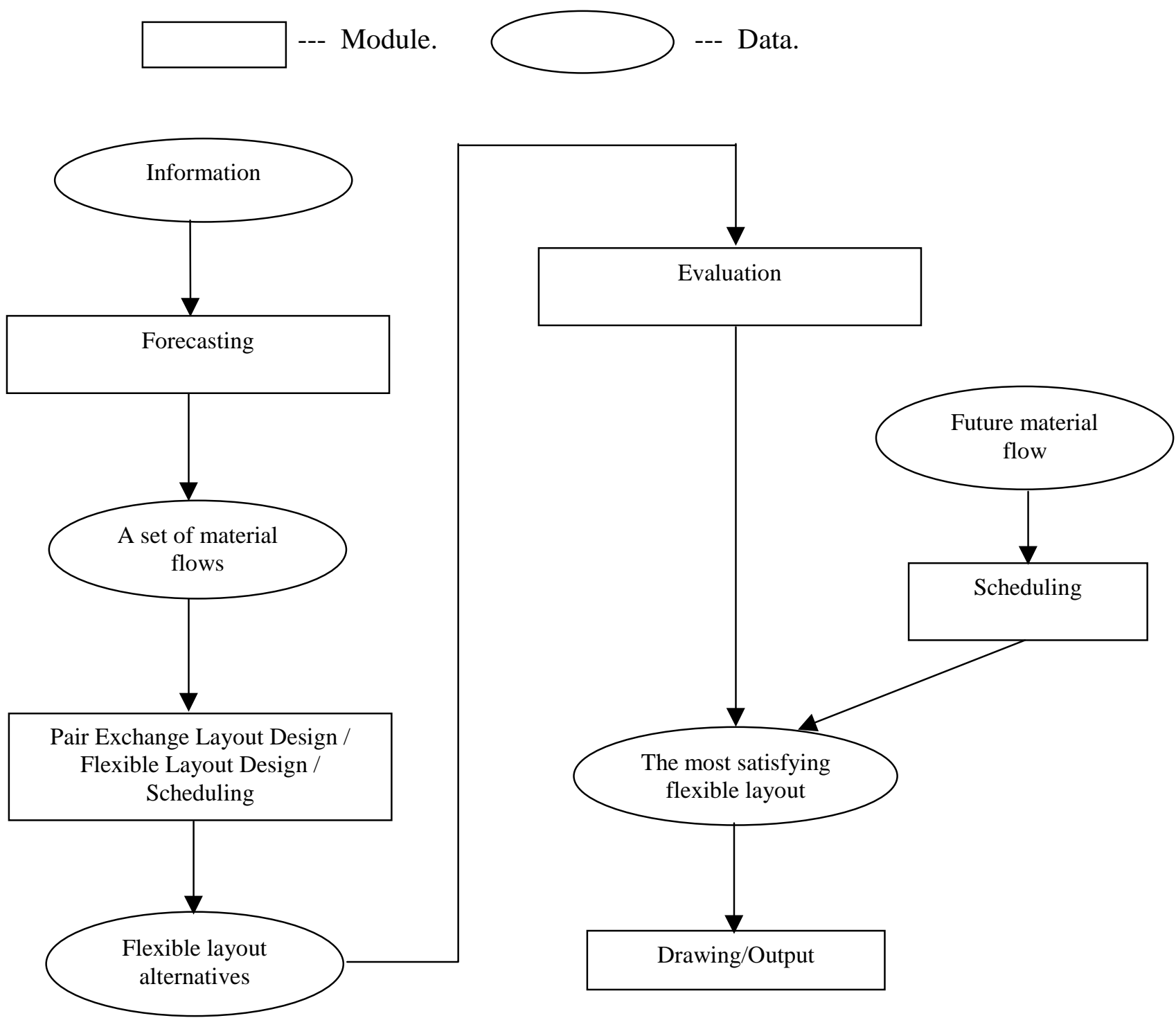

Figure 3-1 Program Flow Diagram 
The system consists of six major modules. The modules are Forecasting, Pair Exchange Layout Design, Flexible Layout Design, Scheduling, Layout Evaluation, and Layout Drawing.

The Forecasting module takes the historical production data as the input. It uses the linear regression method to create future material flows. The user can change these results based on his/her knowledge.

The Pair Exchange Layout Design module takes the Forecasting module's future information output as input. It starts with an initial layout, and then considers all possible two-way department exchanges. The layout is updated according to the best exchange. The process continues until no further exchange can reduce the total material handling cost.

The Flexible Layout Design module takes both the Forecasting module's future information output and the output of the Pair Exchange Layout Design module as input. It uses the flexible layout design algorithm to create several flexible layout alternatives, which shorten the distance among the departments and provide opportunities for rescheduling. Hence, the layout created reduces the total material handling cost and has the capacity to react to disturbances in the future.

The Scheduling module is used to help the Flexible Layout Design module to realize the created layout's flexibility. The module reschedules the material flows according to final layout to shorten the material moving distance among the departments and reduce the total material handling cost. 
The Layout Evaluation module uses probability theory to analyze and evaluate the layout candidates from the Layout Design module. It takes the part's sequence of operations as input and calculates the mean and variance of total material handling cost. The one layout that is the most insensitive to future change is chosen as the most flexible layout. The Layout Drawing module can draw and print out the final layout as well as all other layout candidates.

The above discussion provides a general outline of the flexible layout design system and the computer program structure. The modules of the computer programs have specific algorithms to perform the intended functions. Also, the modules are interconnected with each other. Specific details of the flexible layout design algorithm and each of the modules of the computer program are provided in the following sections and chapters.

\subsection{Forecasting Algorithm}

With the layout design in this paper, that the demand is uncertain is assumed. So we are trying to make better estimates of what will happen in the future in the face of uncertainty. That is why the forecasting function is included in the system.

\subsubsection{Forecasting}

Forecasting is the art and science of predicting future events. It may involve taking historical data and projecting them into the future with some sort of statistical model. Or it may be a subjective or intuitive prediction of the future. Or it may involve a combination of these. Forecasts are usually classified by the future time horizon that they 
cover as following three categories: short-range forecast, medium-range forecast, and long-range forecast. For layout design, medium-range and long-range forecasts are useful.

There are two general approaches to forecasting. One is quantitative analysis; the other is a qualitative approach. Quantitative forecasts employ a variety of statistical models that use historical data to forecast demand. Qualitative forecasts incorporate important factors such as the decision maker's intuition, emotions, personal experiences and value system in reaching a forecast. Some of the quantitative forecasting methods are naive approach, moving averages model, exponential smoothing model and linear regression model.

\subsubsection{Forecasting model}

The linear regression model [Heizer, 1996] is employed in this forecasting module. The formula is

$$
\hat{y} \quad=\mathbf{a}+\mathbf{b x}
$$

where,

$$
\begin{array}{lll}
\hat{\mathrm{y}} & =\quad \text { Estimated value of the dependent variable } \\
\mathrm{a} & =\quad \text { Y-axis intercept } \\
\mathrm{b} & =\quad \text { Slope of the regression line } \\
\mathrm{x} & =\quad \text { Independent variable, which is time here }
\end{array}
$$

By using the least squares method, "a" and "b" can be found as follows: 
$\begin{aligned} \text { b } & =\frac{\sum_{i=1}^{n} x_{i} y_{i}-n \bar{x} \bar{y}}{\sum_{i=1}^{n} x_{i}{ }^{2}-n \bar{x}^{2}} \\ \text { a } \quad & =\bar{y}-b \bar{x}\end{aligned}$

where,

$\begin{array}{lll}\overline{\mathrm{x}} & = & \text { Average of the value of the } \mathrm{x}^{\prime} \mathrm{s} \\ \overline{\mathrm{y}} & = & \text { Average of the value of the } \mathrm{y}^{\prime} \mathrm{s} \\ \mathrm{n} & = & \text { Number of data points }\end{array}$

A $(1-\alpha) 100 \%$ confidence interval for the mean response $\mathrm{E}\left(\mathrm{y}_{\mathrm{h}}\right)$ can be calculated as below:

$\hat{y}_{\mathbf{h}}-\mathbf{t}(\mathbf{1}-\boldsymbol{\alpha} / \mathbf{2} ; \mathbf{n}-\mathbf{2}) \sqrt{\operatorname{MSE}\left(\frac{1}{\mathrm{n}}+\frac{\left(\mathrm{x}_{\mathbf{h}}-\overline{\mathrm{x}}\right)^{2}}{S_{\mathrm{xx}}}\right)}<\mathbf{E}\left(\mathbf{y}_{\mathbf{h}}\right)$

$$
<\quad \hat{y}_{\mathbf{h}}+\mathbf{t}(\mathbf{1}-\boldsymbol{\alpha} / \mathbf{2} ; \mathbf{n}-\mathbf{2}) \sqrt{\operatorname{MSE}\left(\frac{1}{\mathrm{n}}+\frac{\left(\mathrm{x}_{\mathrm{h}}-\overline{\mathrm{x}}\right)^{2}}{\mathrm{~S}_{\mathrm{xx}}}\right)}
$$

where,

$$
\begin{array}{lll}
\mathrm{t}(1-\alpha / 2 ; \mathrm{n}-2) & = & \text { Value of the } \mathrm{t} \text {-distribution with } \mathrm{n}-2 \text { degrees of freedom } \\
\mathrm{S}_{\mathrm{xx}} & =\sum_{i=1}^{n}\left(x_{i}-\bar{x}\right)^{2} \\
\mathrm{MSE} & =\mathrm{SSE} /(\mathrm{n}-2)
\end{array}
$$


SSE

$$
=\quad \sum_{i=1}^{n}\left(y_{i}-\hat{y}_{i}\right)^{2}=\sum_{i=1}^{n}\left(y_{i}-\bar{y}\right)^{2}-b \sum_{i=1}^{n}\left(x_{i}-\bar{x}\right)\left(y_{i}-\bar{y}\right)
$$

There are four steps to compute a forecast:

Step 1: $\quad$ Compute the slope "b" by using the equation (3-2).

Step 2: $\quad$ Compute the y-intercept "a" by using the equation (3-3).

Step 3: $\quad$ Calculate the forecast $\hat{y}$ by using the equation (3-1).

Step 4: Calculate the confidence interval of $\mathrm{E}(\hat{\mathrm{y}})$ by using the equation (3-4).

\subsection{Pair Exchange Layout Design Algorithm}

The flexible layout design algorithm, which handles the uncertain material flow, is a heuristic improvement method. It starts with an initial layout, so the procedure is pathdependent. Different initial layouts can result in different solutions. In order to get a better solution, it is important to have a good starting point. That is why a pair exchange layout design method is used to create a layout, and its result is used as an initial layout.

This method was first used by CRAFT. It considers all possible two-way department exchanges and takes the one that gives the largest material handling cost reduction as the best exchange. The pair exchange method only picks up the best exchange at each iteration. It does not 'look back' or 'look forward' during the search. Such a solution is very likely to be only locally optimal.

The procedure used here is that from an initial layout, all possible two-way department exchanges will be considered. For each exchange, a temporary layout is built 
and all departments' center points are recalculated. These actual center points are used to calculate the total material handling cost. After all exchanges' total material handling costs are calculated, the exchange that has the smallest total material handling cost is the best exchange. If the smallest total material handling cost is less than the previous layout's total material handling cost, the layout is modified according to this exchange. The procedure continues until no further reduction in material handling cost can be obtained.

The method used here is a CRAFT-like algorithm, but it has some improvement. When comparing an exchange, CRAFT assumed that after two departments make the exchanges, their central points are the same as the previous departments. CRAFT used the estimated position of departments to calculate the material handling cost. If the departments differ in size, the estimated central points may deviate significantly from their correct locations. As a result, the actual reduction in the material handling cost may be overestimated or underestimated. The pair exchange layout design algorithm implemented here uses the actual central points for material handling calculation, so the result is more accurate.

CRAFT assumed that the material flow is deterministic, so it had only one flow chart. The algorithm implemented here considers the material flow as uncertain. Users can give a distribution of the flow chart.

CRAFT considered only one piece of material handling equipment for the material flow between two departments. That means that there is only one unit cost between two departments. The algorithm implemented here divides the flows according to different 
materials. Each material can have its own material handling equipment. So users can have more than one unit cost between two departments.

\subsection{Flexible Layout Design Algorithm}

\subsubsection{Introduction}

In this section, the flexible layout design algorithm is described in detail. This algorithm considers change in material flow. The material flow is assumed to be stochastic and uncertain. Under this condition, several possible material flows are forecasted by the forecasting model. The flexible layout design algorithm takes these possible material flows as input data, then splits the departments and reschedules the flows among departments. The distances among the departments are thus reduced. Then the total material handling cost is reduced. Furthermore, splitting the departments provides the opportunity to reschedule material flows among the departments. When there are unexpected material flows in the future, by rescheduling them we can still keep the total material handling cost low with this layout. The algorithm makes the layout flexible to disturbance in the future. Some case studies presented in Chapter 5 show significant cost reduction.

While this algorithm is designed for uncertain material flow condition, it is also a good design method for deterministic and known material flow condition. It can still reduce the total material handling cost by shortening the distance among the departments. One case study presented in Chapter 5 shows this kind of cost reduction. 
Furthermore, although this algorithm is dedicated to create a layout that is flexible to material flow change and doesn't need to be rearranged, it can also be used for dynamic layout design. For each period, one layout is created by this algorithm. By shortening the distance among the departments, it can also get a good result.

\subsubsection{Problem Statement and Modeling}

Here the problem is to arrange " $\mathrm{m}$ " departments with different areas into a rectangular plant building to minimize the total expected material handling cost given several possible future material flows. The material flows can have the different cost per unit distance. The model is presented below:

$$
\operatorname{Min} \mathbf{z}=\sum_{i=1}^{m} \sum_{j=1}^{m} \sum_{k=1}^{l_{i j}} \overline{\mathbf{f}}_{\mathbf{i j k}} \mathbf{c}_{\mathbf{i j k}} \mathbf{d}_{\mathbf{i j}}
$$

Where

$$
\begin{array}{ll}
\mathrm{z} & =\text { total material handling cost. } \\
\overline{\mathrm{f}_{\mathrm{ijk}}} \quad= & \text { expected flow of material } \mathrm{k} \text { from department } \mathrm{i} \text { to department } \mathrm{j} . \\
\mathrm{c}_{\mathrm{ijk}} & =\text { cost of moving a unit load of material } \mathrm{k} \text { one unit distance from } \\
& \text { department } \mathrm{i} \text { to department } \mathrm{j} . \\
\mathrm{d}_{\mathrm{ij}} & =\text { distance from department } \mathrm{i} \text { to } \mathrm{j} . \\
\mathrm{m} & =\text { number of departments. } \\
\mathrm{l}_{\mathrm{ij}} & =\text { number of types of materials from department } \mathrm{i} \text { to } \mathrm{j} .
\end{array}
$$




\subsubsection{Input Data}

The input data are

- Building dimensions (Width and length).

- Each department's area.

- Material handling equipment information (Moving cost per unit distance).

- Material handling equipment and material relationship (Which piece of material handling equipment is used for a specific material and how many units per trip).

- Quantity of material flow between two departments.

Apart from these data, the user also needs to give some design parameters:

- Distance definition (Rectilinear or Euclidean).

- Number of bands in the layout.

- Initial layout.

\subsubsection{Flexible Layout Design Procedures}

The procedure flow chart is shown in Figure $3-2$. 


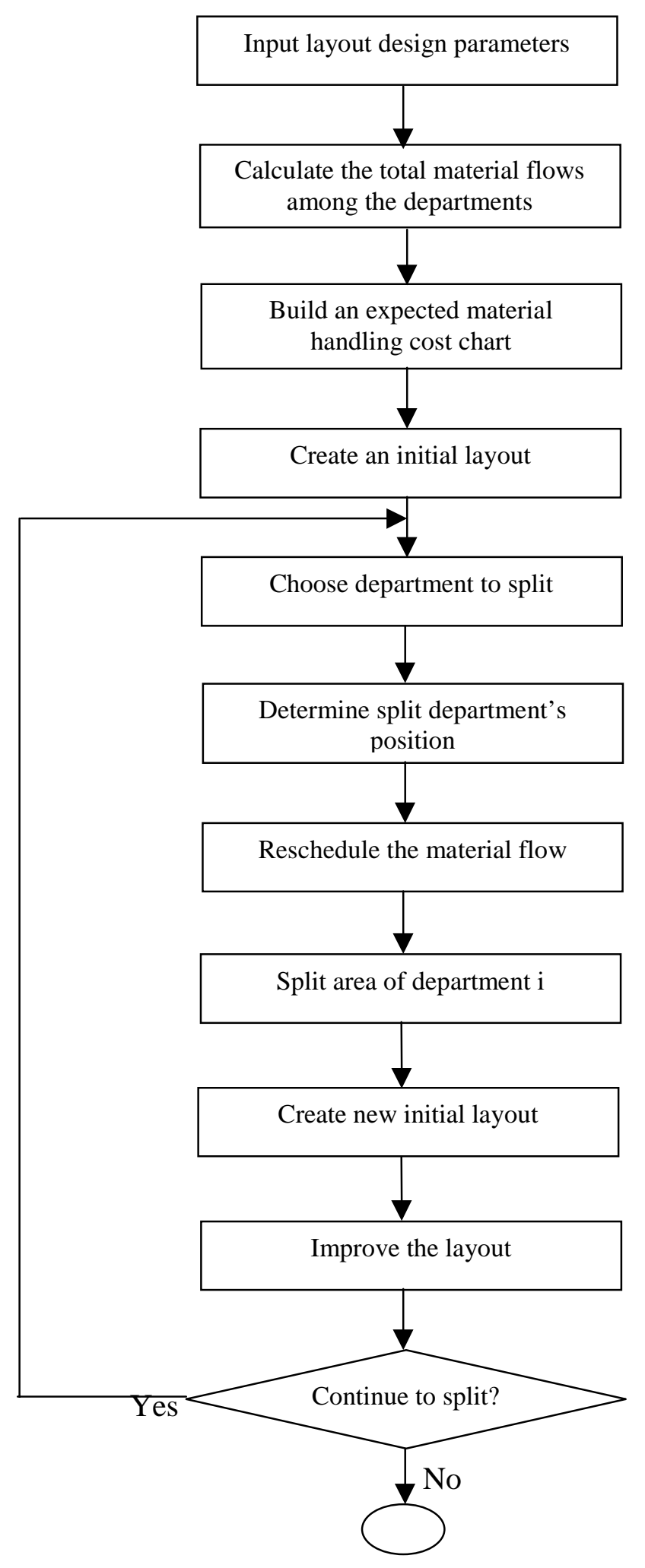

Figure 3-2 Procedure Flow Chart 


\subsubsection{Step 1: Calculate the total material flows among the departments}

- There could be more than one material flow between two departments. The materials could be handled by different equipment. So the material handling cost is calculated to represent all these effects of different material flows:

$\operatorname{MHCost}_{\mathrm{ij}}=\sum_{k=1}^{l_{i j}} \mathbf{c}_{\mathrm{ijk}} \mathbf{f}_{\mathrm{ijk}}+\sum_{k=1}^{l_{j i}} \mathbf{c}_{\mathrm{jik}} \mathbf{f}_{\mathbf{j i k}}$

Where,

MHCost $_{\mathrm{ij}}=$ Material handling cost per unit distance between departments $\mathrm{i}$ and $\mathrm{j}$.

$\mathrm{c}_{\mathrm{ijk}}\left(\mathrm{c}_{\mathrm{jik}}\right)=$ Cost of moving a unit load of material $\mathrm{k}$ from department i to $j(j$ to i)

$\mathrm{f}_{\mathrm{ijk}}\left(\mathrm{f}_{\mathrm{jik}}\right) \quad=\quad$ Flow of material $\mathrm{k}$ from department $\mathrm{i}$ to $\mathrm{j}$ ( $\mathrm{j}$ to $\mathrm{i}$ )

$1_{\mathrm{ij}}\left(l_{\mathrm{ji}}\right) \quad=\quad$ Number of types of materials from department $\mathrm{i}$ to $\mathrm{j}$ ( $\mathrm{j}$ to $\mathrm{i}$ )

After MHCost ${ }_{\mathrm{ij}}$ is calculated, a material handling cost per unit distance chart is built for each possible flow set.

\subsubsection{Step 2: Build an expected material handling cost chart}

- Calculate an expected material handling cost per unit distance chart. If the probability is unknown for each flow set, it is assumed to have a uniform distribution. 


\subsubsection{Step 3: Create an initial layout}

- Use the Pair Exchange Layout Design algorithm to create an initial layout.

\subsubsection{Step 4: Choose departments to split}

- List all pair departments that are not adjacent to each other. Calculate the total non-adjacent material handling cost per unit distance for each department.

$$
\text { Non_MHCost } t_{\mathbf{i}} \quad=\quad \sum_{\mathrm{k}=1}^{\mathrm{n}_{\mathrm{i}}} \text { MHCost }_{\mathbf{i k}}
$$

Where

Non_MHCost $\mathrm{i}_{\mathrm{i}} \quad=$ Non-adjacent material handling cost per unit distance for department i

MHCost $_{\mathrm{ik}}=$ Material handling cost per unit distance between departments $\mathrm{i}$ and $\mathrm{k}$ which are not adjacent

$\mathrm{n}_{\mathrm{i}} \quad=$ Number of departments that are not adjacent with department i

- Select the department (i) that has the greatest non-adjacent material handling cost per unit distance. This department is chosen to be split. It is split to departments $i_{i}$ and $i_{k}$.

\subsubsection{Step 5: Determine split department's position}

- List department i's non-adjacent material handling cost per unit distance with each department. 
- The department $(\mathrm{k})$ that has the most non-adjacent material handling cost per unit distance with department $\mathrm{i}$ will be selected as the referred position.

- Department $i_{i}$ remains in department i's position. Department $i_{k}$ will be inserted next to department $\mathrm{k}$. This is done in order to create a good initial layout.

\subsubsection{Step 6: Reschedule the material flow}

- For each department that has flows with department i, calculate its distance from departments $\mathrm{i}$ and $\mathrm{k}$. Department k's position is used to estimate the position of department $i_{k}$, which will be inserted next to it.

- If the distance is shorter for $\mathrm{i}_{\mathrm{i}}$, the flow is assigned to $\mathrm{i}_{\mathrm{i}}$. Otherwise the flow is assigned to $i_{k}$.

- If the distance is almost the same, assign this flow to balance of $i_{i}$ and $i_{k}$ 's total non-adjacent material handling cost per unit distance.

- The flow between $i_{i}$ and $i_{k}$ is zero.

- Change the material handling cost per unit distance chart by using the above information.

\subsubsection{Step 7: $\quad$ Split area of department $i$}

- Calculate total flows for $\mathrm{i}, \mathrm{i}_{\mathrm{i}}$ and $\mathrm{i}_{\mathrm{k}}$.

- $\operatorname{Area}\left(\mathrm{i}_{\mathrm{i}}\right)=\left[\operatorname{Flows}\left(\mathrm{i}_{\mathrm{i}}\right) / \operatorname{Flows}(\mathrm{i})\right] * \operatorname{Area}(\mathrm{i})$

$$
\operatorname{Area}\left(\mathrm{i}_{\mathrm{k}}\right)=\left[\operatorname{Flows}\left(\mathrm{i}_{\mathrm{k}}\right) / \operatorname{Flows}(\mathrm{i})\right] * \operatorname{Area}(\mathrm{i})
$$




\subsubsection{Step 8: New initial layout}

- Take layout from step 3 as the basic layout.

- Department $\mathrm{i}_{\mathrm{i}}$ remains in department i' position.

- Department $\mathrm{i}_{\mathrm{k}}$ is inserted next to department $\mathrm{k}$. Insert department $\mathrm{i}_{\mathrm{k}}$ to department k's left side and right side respectively to get two new initial layouts.

\subsubsection{Step 9: Improve the layout}

- Use the Pair Exchange Layout Design algorithm again to find the 'best' layout.

\subsubsection{Step 10: Continue to split}

- Go back to step 4 to select another candidate department to split, or

- If satisfied with the layout, stop.

\subsubsection{Step 11: Schedule future material flows}

When the future material flows are finally known, they are rescheduled to take advantage of this flexible layout to reduce the distances among the departments.

\subsection{Evaluation Algorithm}

The evaluation algorithm developed here utilizes probabilistic methods to evaluate layouts. It has the following assumptions:

1) Each part has a sequence of operations. An operation is finished by one department. During the operations, the quantity of parts doesn't change. 
2) Parts' sequences of operations are independent of each other. The demands of parts are independent of each other.

3) The sequence of operations is feasible. The schedule of one sequence of operations doesn't affect other schedules.

4) The demands of parts are uncertain. They are random variables. They have normal distribution. Their mean and variance are known. The normal distribution assumption can be relaxed later on.

Here the sequence of operations is considered instead of from-to flow because fromto flows are not independent of each other so it is difficult to consider their distribution. The sequence of operations can be converted to from-to flow. Therefore users can use either from-to flow information or sequence of operations to design a layout. The system provides more options to users.

In terms of a part's sequence of operations, we have the following equation for calculating the total material handling cost:

\begin{tabular}{|c|c|c|}
\hline MHCost & $=$ & $\sum_{i} \sum_{j, k}\left(\mathbf{q}_{\mathbf{i}} / \mathbf{n}_{\mathbf{i j k}}\right) * \mathbf{c}_{\mathbf{i j k}} * \mathbf{d}_{\mathbf{j k}}$ \\
\hline \multicolumn{3}{|l|}{ Where } \\
\hline MHCost & $=$ & Total Material Handling Cost \\
\hline $\mathrm{q}_{\mathrm{i}}$ & $=$ & Quantity of part $\mathrm{i}$ in sequence of operations \\
\hline $\mathrm{n}_{\mathrm{ijk}}$ & $=$ & Quantity of part i per trip from department $\mathrm{j}$ to $\mathrm{k}$ \\
\hline $\mathrm{c}_{\mathrm{ijk}}$ & $=$ & Unit cost for part $\mathrm{i}$ from department $\mathrm{j}$ to $\mathrm{k}$ \\
\hline$d_{\mathrm{jk}}$ & $=$ & Distance from department $\mathrm{j}$ to $\mathrm{k}$ \\
\hline
\end{tabular}


Within the sequence of operations, the part is the same so the material handling equipment is the same. Then the unit cost is the same. $c_{\mathrm{ijk}}$ can be simply expressed by $c_{\mathrm{i}}$. $n_{\mathrm{ijk}}$ can be simply expressed by $\mathrm{n}_{\mathrm{i}}$. Equation (3-8) becomes

$$
\operatorname{MHCost}=\sum_{i}\left[\left(\mathbf{c}_{\mathrm{i}} / \mathbf{n}_{\mathbf{i}}\right) *\left(\sum_{j, k} \mathbf{d}_{\mathbf{j k}}\right) * \mathbf{q}_{\mathbf{i}}\right]
$$

Here $c_{i}, n_{i}$ and $d_{j k}$ are constant. $q_{i}$ is a random variable and independent of each other. So MHCost is also a random variable and a linear combination of all of $\mathrm{q}_{\mathrm{i}}$. Since all of $\mathrm{q}_{\mathrm{i}}$ is normally distributed, MHCost is also normally distributed. The mean $\mu$ and variance $\sigma^{2}$ of MHCost can be calculated as following:

$$
\begin{aligned}
& \boldsymbol{\mu}(\mathbf{M H C o s t})=\sum_{i}\left[\left(\mathbf{c}_{\mathbf{i}} / \mathbf{n}_{\mathbf{i}}\right) *\left(\sum_{j, k} \mathbf{d}_{\mathbf{j k}}\right) * \boldsymbol{\mu}\left(\mathbf{q}_{\mathbf{i}}\right)\right] \\
& \sigma^{2}(\text { MHCost })=\sum_{i}\left\{\left[\left(\mathbf{c}_{\mathbf{i}} / \mathbf{n}_{\mathbf{i}}\right) *\left(\sum_{j, k} \mathbf{d}_{\mathbf{j k}}\right)\right]^{2} * \sigma^{2}\left(\mathbf{q}_{\mathbf{i}}\right)\right\}
\end{aligned}
$$

$\mu\left(\mathrm{q}_{\mathrm{i}}\right)$ and $\sigma^{2}\left(\mathrm{q}_{\mathrm{i}}\right)$ can be estimated from historical data of $\mathrm{q}_{\mathrm{i}}$ :

$$
\begin{aligned}
& \hat{\mu}\left(\mathbf{q}_{\mathbf{i}}\right)=\bar{q}_{i}=\sum_{j=1}^{n} \mathbf{q}_{\mathbf{i j}} / \mathbf{n} \\
& \hat{\sigma}^{2}\left(\mathbf{q}_{\mathbf{i}}\right)=\sum_{j=1}^{n}\left(\mathbf{q}_{\mathbf{i j}}-\bar{q}_{i}\right)^{2} /(\mathbf{n}-\mathbf{1})
\end{aligned}
$$

where,

$$
\begin{array}{lll}
\hat{\mu}\left(\mathrm{q}_{\mathrm{i}}\right) & =\text { Point estimate of } \mu\left(\mathrm{q}_{\mathrm{i}}\right) \\
\hat{\sigma}^{2}\left(\mathrm{q}_{\mathrm{i}}\right) & = & \text { Point estimate of } \sigma^{2}\left(\mathrm{q}_{\mathrm{i}}\right) \\
\mathrm{q}_{\mathrm{ij}} & = & \text { Demand of part } \mathrm{i} \text { at period } \mathrm{j}
\end{array}
$$




$$
\begin{array}{lll}
\bar{q}_{i} & =\quad \text { Average of the value of the } \mathrm{q}_{\mathrm{ij}} \\
\mathrm{n} & =\quad \text { Number of periods }
\end{array}
$$

Since MHCost is normally distributed, there is 0.6826 probability that $\mu-\sigma<$ MHCost $<\mu+\sigma ; 0.9544$ probability that $\mu-2 \sigma<$ MHCost $<\mu+2 \sigma$; and 0.9974 probability that $\mu-3 \sigma<$ MHCost $<\mu+3 \sigma$. So after calculating the mean and variance of total material handling cost, we can know the range of the total material handling cost of each layout under uncertain situations. The range is the criterion for evaluating the flexible layout.

Hence, not only the mean of the total material handling cost is used for evaluation, which is very common, but also the variance of the total material handling cost can be used for evaluation. This new evaluation tool can provide more accurate information for comparing layouts under uncertainty. The layout with less variance, i.e., more narrow range, is the more flexible layout.

We can still evaluate layouts by using mean and variance without normal distribution assumptions. Let's say MHCost is not necessarily normally distributed. Then Chebyshev's Theorem [Walpole, 1993] can be used. According to that theorem, there is more than 0.75 probability that $\mu-2 \sigma<$ MHCost $<\mu+2 \sigma$; and 0.888 probability that $\mu$ $-3 \sigma<$ MHCost $<\mu+3 \sigma$. These probabilities are not better than those with normal distribution but are still useful for evaluating the flexibility of layouts. 


\subsection{Conclusion}

In this chapter a general outline of the flexible layout design system and the computer program structure were discussed, and the major algorithms were described in detail. Among them the forecasting algorithm was applied to layout design. The pair exchange layout design algorithm is an improvement over the existing one. It is used to create an initial layout. The flexible layout design algorithm is a new algorithm in layout design. It is used to create flexible layout with less total material handling cost. Evaluation algorithm is also a new algorithm that applies the probability theory to layout evaluation. 


\section{COMPUTER PROGRAM DESIGN}

\subsection{Overall Program Structure}

A computer-based system was developed to implement the flexible layout design algorithm and other functions related to layout design. The system consists of ten subsystems: (1) Forecasting; (2) Initialization; (3) Pair exchange layout design algorithm; (4) Placing layout; (5) Drawing layout; (6) Flexible layout design algorithm; (7) Reschedule flow; (8) Layout Evaluation; (9) Re-sequencing the operations; (10) Converting sequence into from-to flow. Figure 4-1 shows the overall program structure and information relationships.

The forecasting sub-system is a medium and long-range forecast system. It is a demand forecast that projects demand for a company's products. The linear regression method is used in the system. The user needs to enter historical data that are the input to the forecast algorithm. Estimates of future demands are the outputs that are used in the initialization model.

The initialization sub-system takes into account the future demands, material and the material handling system's cost information. It turns all this information into a from-to flow chart in which the cost is also considered. The from-to flow chart is used in the layout design model. 
(1) Forecasting

(Table) Forecasting

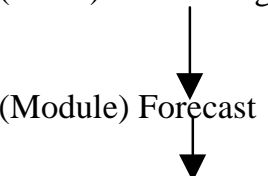

(Table) Quantity_per_month

(2) Initialization

(Table) Quantity_per_month $\leftarrow$

(Module) Fromto

(Module) Expected_fromto_queryToTable

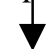

(Table) Expected fromto

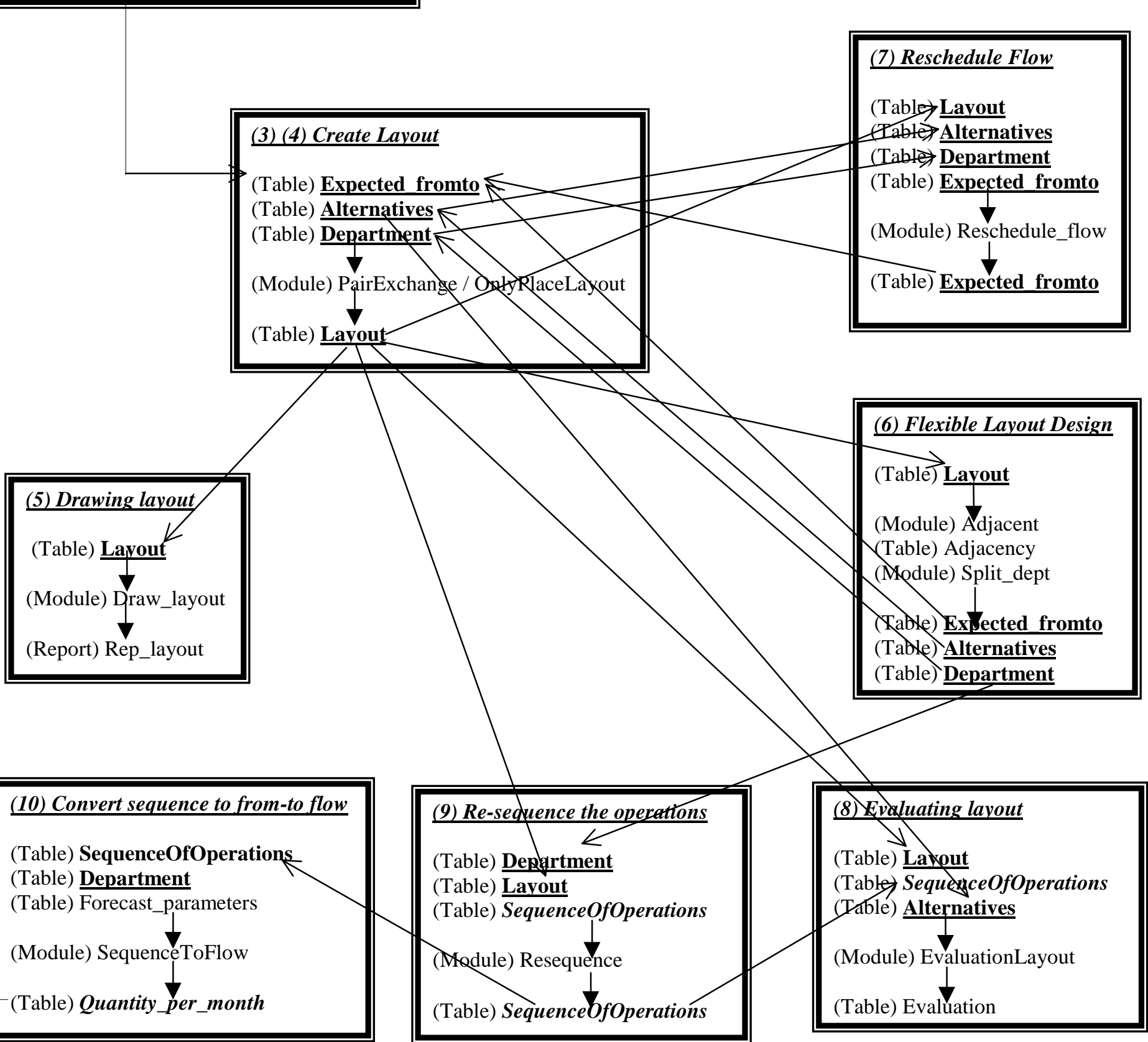

Figure 4-1 Program Structure 
The pair exchange layout design sub-system uses the pair exchange algorithm to create a layout. It starts with an initial layout, then considers all possible two-way department exchanges and identifies the best exchange, that is, the one that yields the greatest reduction in the material handling cost. Once the best exchange has been identified, the sub-system updates the layout according to the best exchange. The process continues until no further reduction in material handling cost can be obtained. Because the algorithm picks only the best exchange at each iteration, it is a local optimal procedure.

The placing layout sub-system doesn't have any algorithm. It just places the departments into the layout according to given order and calculates the material handling cost. It is a simple layout evaluation. The material handling cost is used as the evaluation criterion for layouts created by different methods.

The flexible layout design sub-system implements the flexible layout design algorithm. It uses the layout created by the pair exchange layout design sub-system as the initial layout, then splits several departments to form a hybrid layout that combines the job shop layout and cellular layout. By shortening the distances between departments and providing opportunities for rescheduling, the layout created by this algorithm is efficient and has the capacity to react to disturbances caused by future events. That is, it is a flexible layout.

The reschedule flow sub-system is used to help the flexible layout design sub-system to make the created layout flexible. Given the uncertainty of material flow, future 
material flows may be very different from any flows used for creating the layout. This module reschedules the material flow according to the final layout to shorten the distances between the departments and reduce the total material handling cost. This makes the layout efficient and flexible. The sub-system only makes sense after the flexible layout design sub-system has been used. The latter makes rescheduling possible.

The drawing layout sub-system is used to output a layout. The material handling cost and split status are also given with the layout.

The layout evaluation sub-system provides a tool to evaluate a layout. It uses the parts' sequence of operations as input and assumes that the quantity of parts is a random variable with certain distribution. Then the material handling cost's mean and variance are calculated and used as criteria of evaluation.

Re-sequencing the operations sub-system takes the parts' original sequence of operations as input, then according to split status, re-arranges the parts' sequence of operations in order to take advantage of split departments to reduce the material handling cost.

The converting sequence into from-to flow sub-system converts the parts' sequence of operations into flows between two departments. Then these from-to flows can be used at the layout design model.

The system is implemented by using Microsoft Access $97^{\circledR}$ and Visual Basic ${ }^{\circledR}$. 


\subsection{Forecasting Module}

The Forecasting module involves a linear regression model to generate the forecast. The relationship of this module is shown in Figure 4-2.

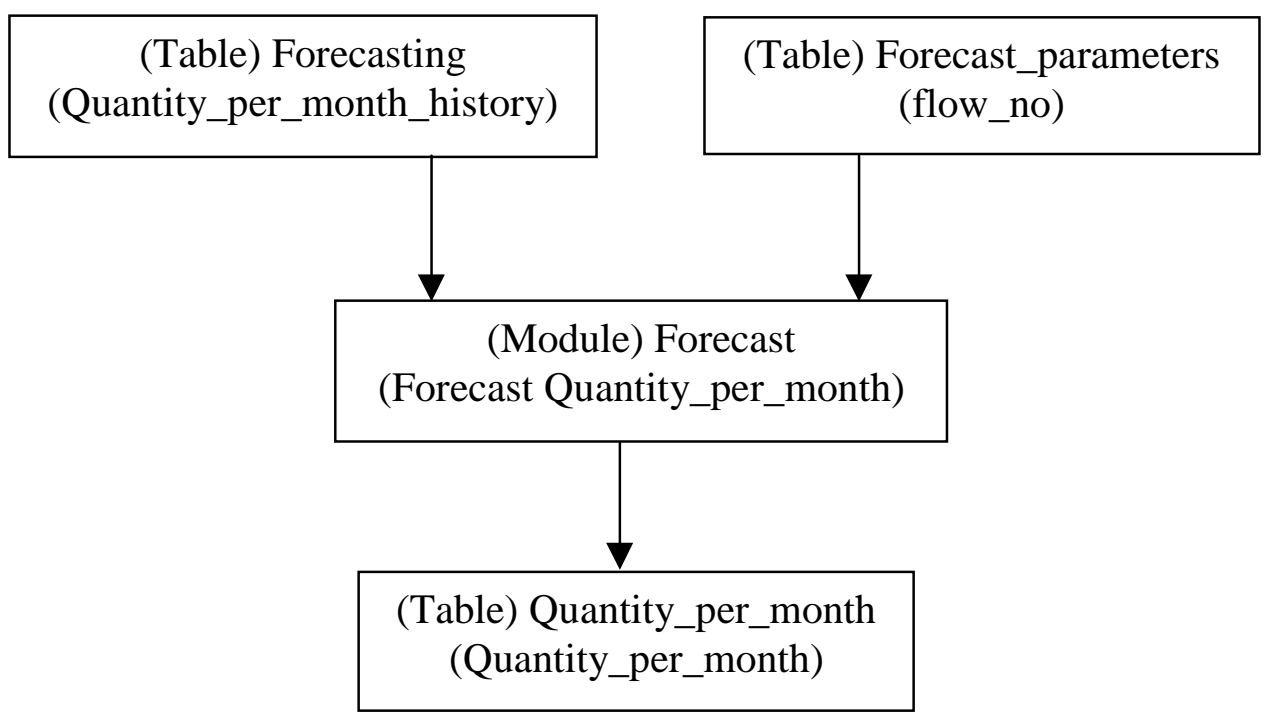

Figure 4-2 Forecasting Module Relationship

Users first need to input the historical data regarding the quantity of each material flow between two departments, which are stored in the table Forecasting. The module Forecast utilizes the above information and generates a forecast by using the linear regression method. The program flow of the module Forecast is shown in Figure 4-3. The result of forecasting, which is the future quantity of each material flow between two departments, is stored in the table Quantity_per_month. This information will be used by the layout design module. 


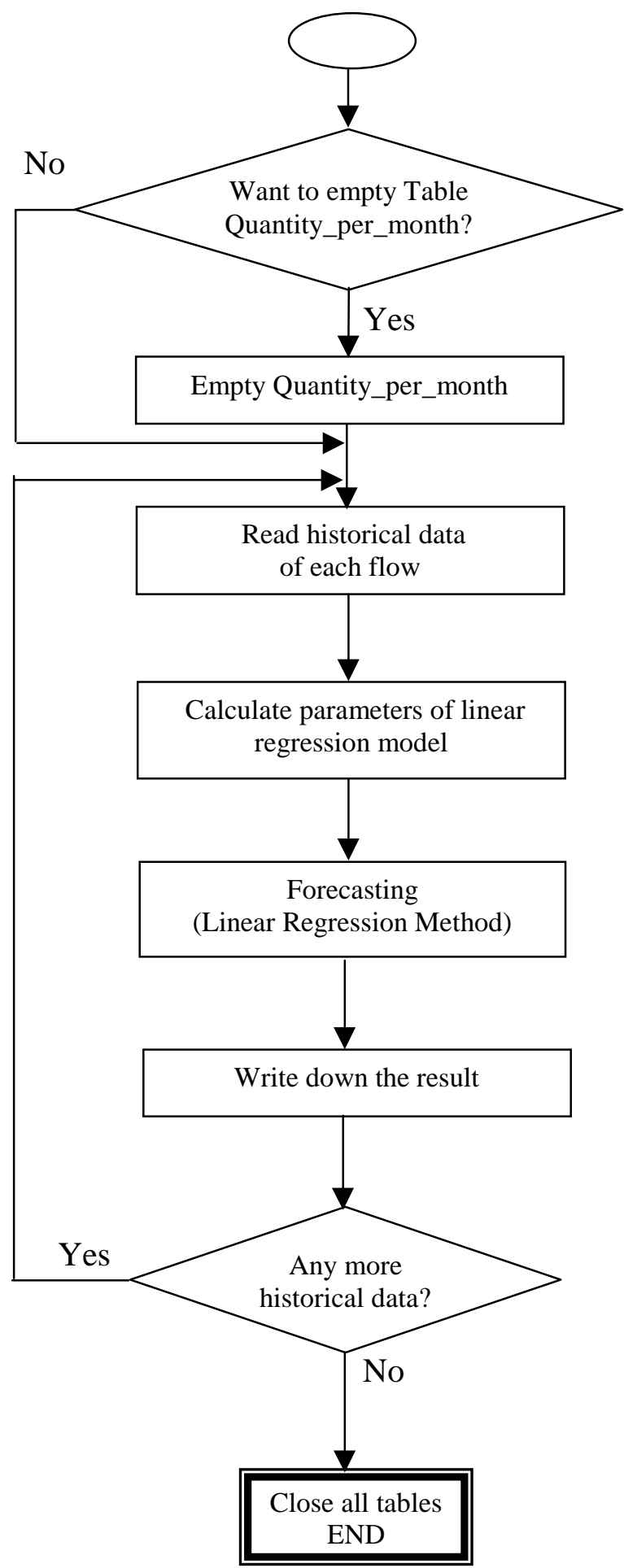

Figure 4-3 Program Flow of Forecast Module 
For example, the quantities of material 1 moved between departments A and B during the last nine periods are $12,17,20,19,24,26,31,32,36$. These data are input by the user and stored in the table Forecasting. The linear regression method is used, and the forecasting value for the next period is calculated as 38.24. 38.24 is stored in the table Quantity_per_month as the future quantity of material 1 moved between departments A and $\mathrm{B}$.

\subsection{Initialization Module and Pair Exchange Layout Design Module}

These two modules are used to create an initial layout and improve the layout with the Flexible Layout Design module. The relationships of these two modules is shown in Figure 4-4.

The pair exchange layout algorithm uses a from-to flow chart as the input information. Usually a from-to flow chart does not exist. What the plant has is information about material handling equipment, raw material, material handling equipment and raw material relationships, etc. Therefore, an initialization module was developed to build from-to flow charts.

Users should first define a material handling system which includes information about the moving cost per unit distance for each piece of material handling equipment, which material handling equipment is used for a specific material and how many 


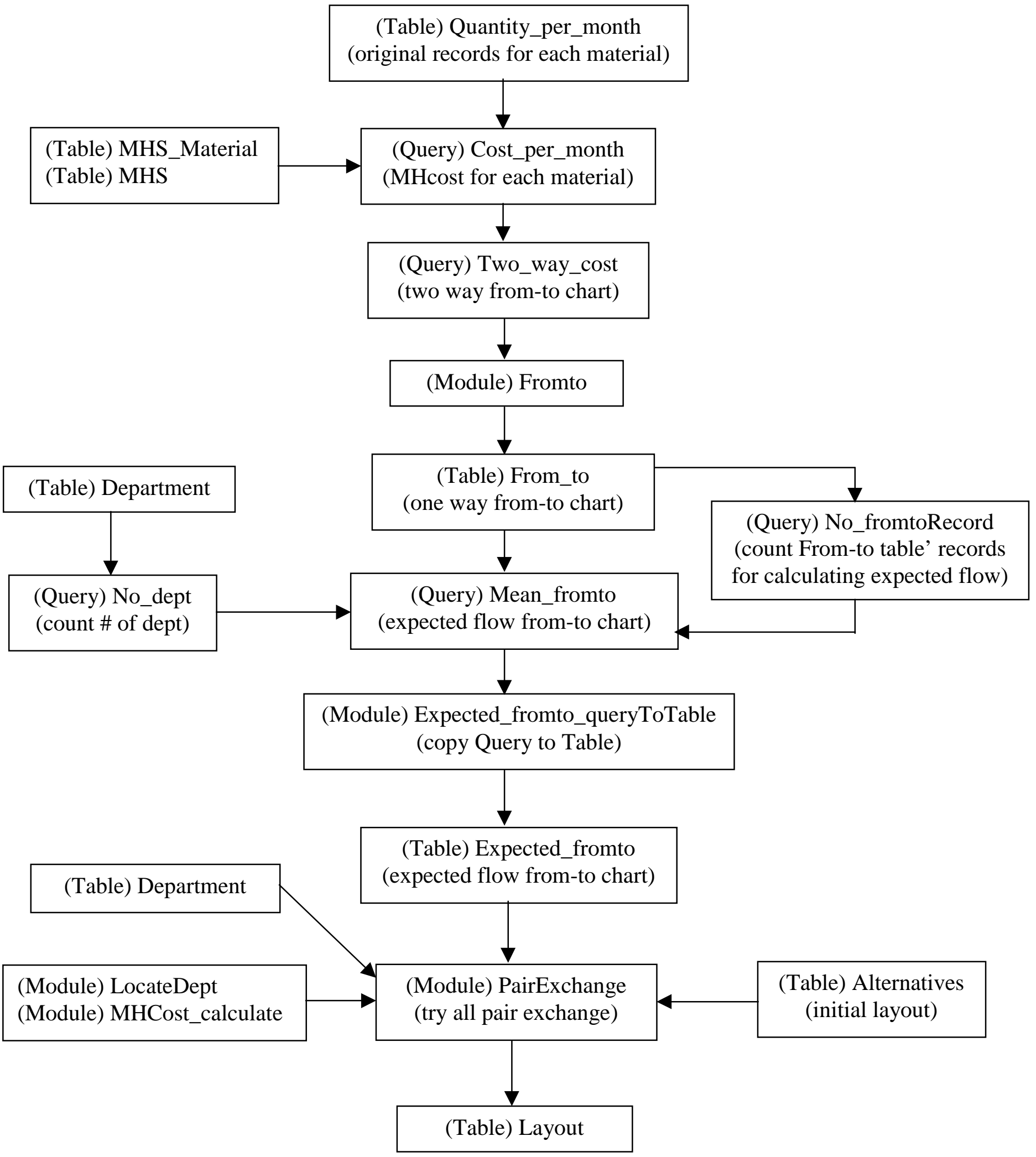

Figure 4-4 Pair Exchange Layout Design Module Relationship 
units per trip. All above information is stored in two tables: MHS and MHS_material. Other data need to be input by the user are building width and length, each department's area, distance definition, number of bands in the layout and initial layout. These data are stored in the table Department and Alternatives. The quantity of material flow can be input by the user or can be the result of the forecasting. It is stored in the table Quantity_per_month. The initialization module combines the flows of different materials based on the moving cost for each possible material flow. Then it calculates the expected material flow from all possible material flows. The result is stored in the table Expected_fromto. This result is used as the from-to chart for the layout design. In terms of this from-to chart, all pair exchanges are tested and the best one is selected for real exchange. This process continues until no improvement can be obtained by further exchange. The final layout is stored in the table Layout. Figure 4-5 shows the program flow of the module PairExchange.

\subsection{Flexible Layout Design Module}

This module is a computer implementation of the algorithm described in Chapter 3. An initial layout from the result of the pair exchange module is checked to see the adjacent status of each department. Adjacency depends on the department's position in layout. Material flow is a weight factor for the adjacency. If two departments are adjacent to each other, the distance between them should be short. The material handling cost is expressed as

MHCost $=($ Flow $) *($ Cost/trip/unit distance $) *($ Distance $)$ 


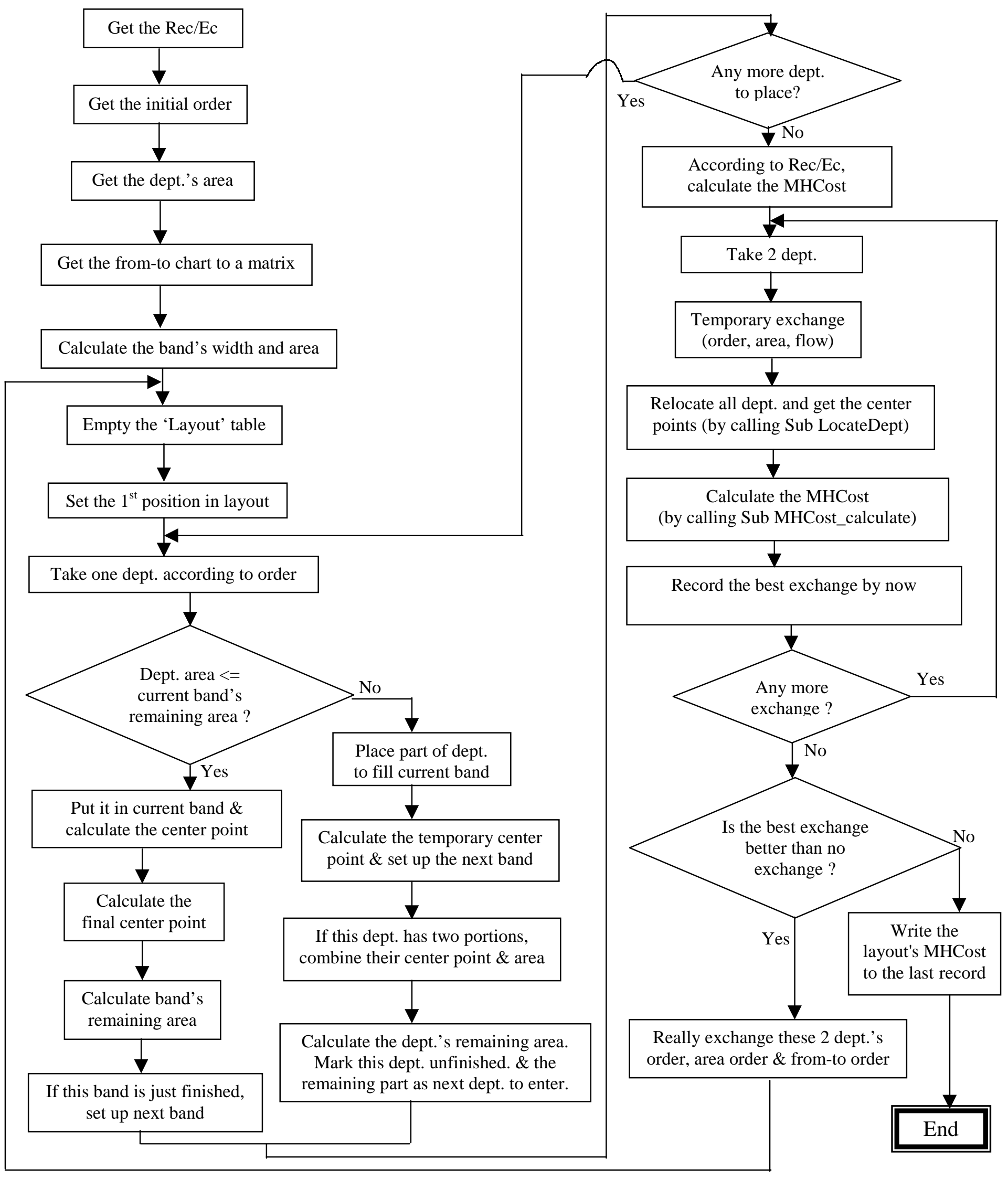

Figure 4-5 Program Flow of Module PairExchange 
Therefore, when the distance goes down, the material handling cost goes down. The layout is good. If not, the algorithm takes their adjacent relationship as a possible improvement opportunity. All departments are checked, and all non-adjacent relationships are taken into account. From equation (4-1) we can see that the material handling cost is proportional to (material flow) * (unit cost). If this portion is large, in which case we cannot control it, we prefer to reduce the distance first. Otherwise the material handling cost will go up significantly. So this portion is used as a weight factor for non-adjacent relationships. Among those departments that have non-adjacent relationships, the one that has the maximum (material flow) * (unit cost) will be considered for a split by the algorithm.

Figure 4-6 shows the information relationships of this module. From the initial layout of departments, the adjacency of departments is analyzed by the module Adjacent. The information about non-adjacent departments is stored in the table Adjacency. Microsoft Access Query is used to select a department that has the total maximum non-adjacent (material flow) * (unit cost) with other departments. This department is to be split. Microsoft Access Query is also used to select a department that has the maximum nonadjacent (material flow) * (unit cost) with the split department. This department is used to locate one portion of the split department. The Module Split_dept splits the selected department and locates the new portion. It then re-schedules the material flows among the new portions of the split departments and other departments. The rule of rescheduling is to assign the material flows to one department and one of the portions of the split department in order to have a shorter distance. This layout is an improvement to the one created by the pair exchange method. Furthermore, this layout is ready to use the 


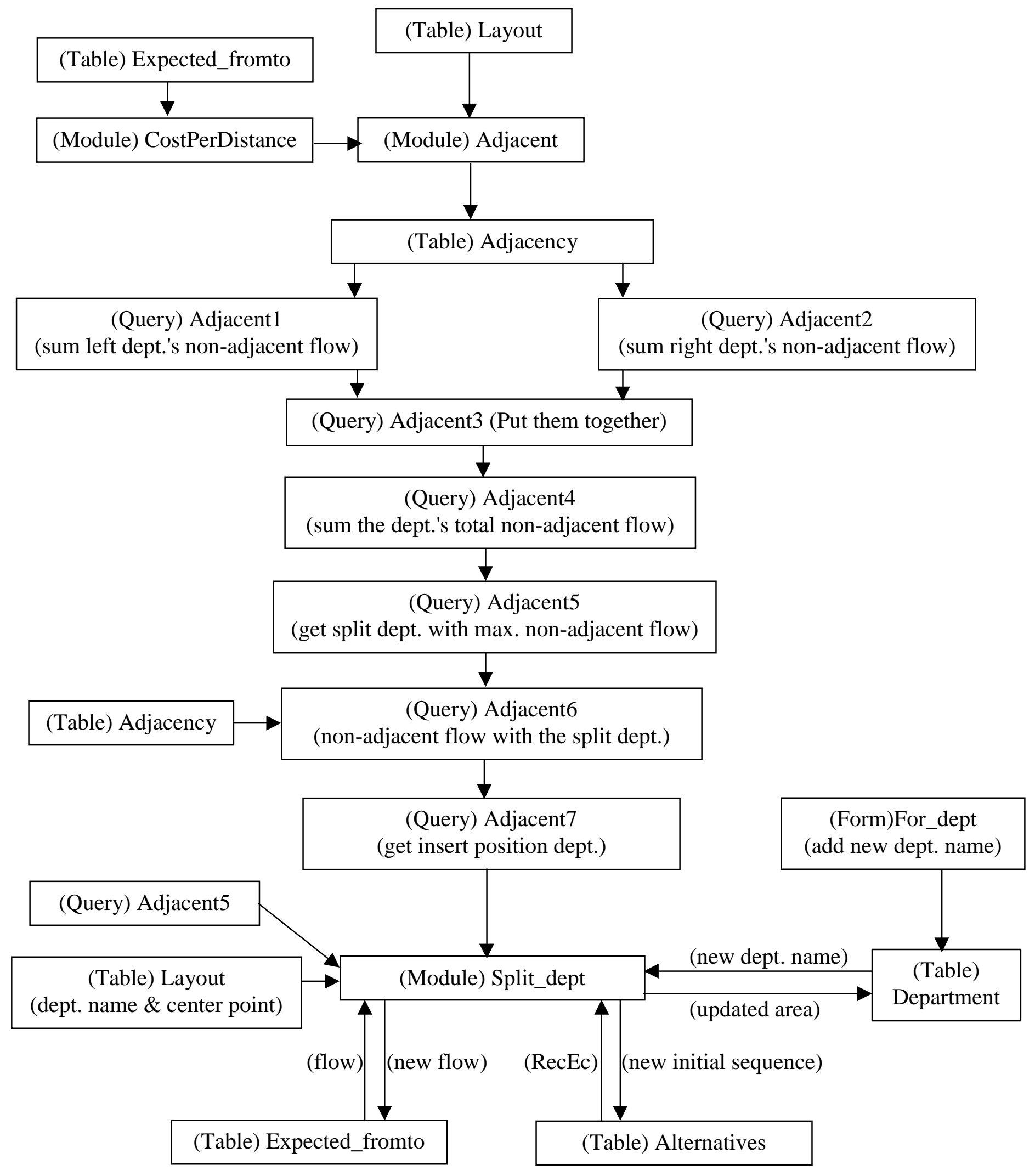

Figure 4-6 Flexible Layout Design Module Relationships 


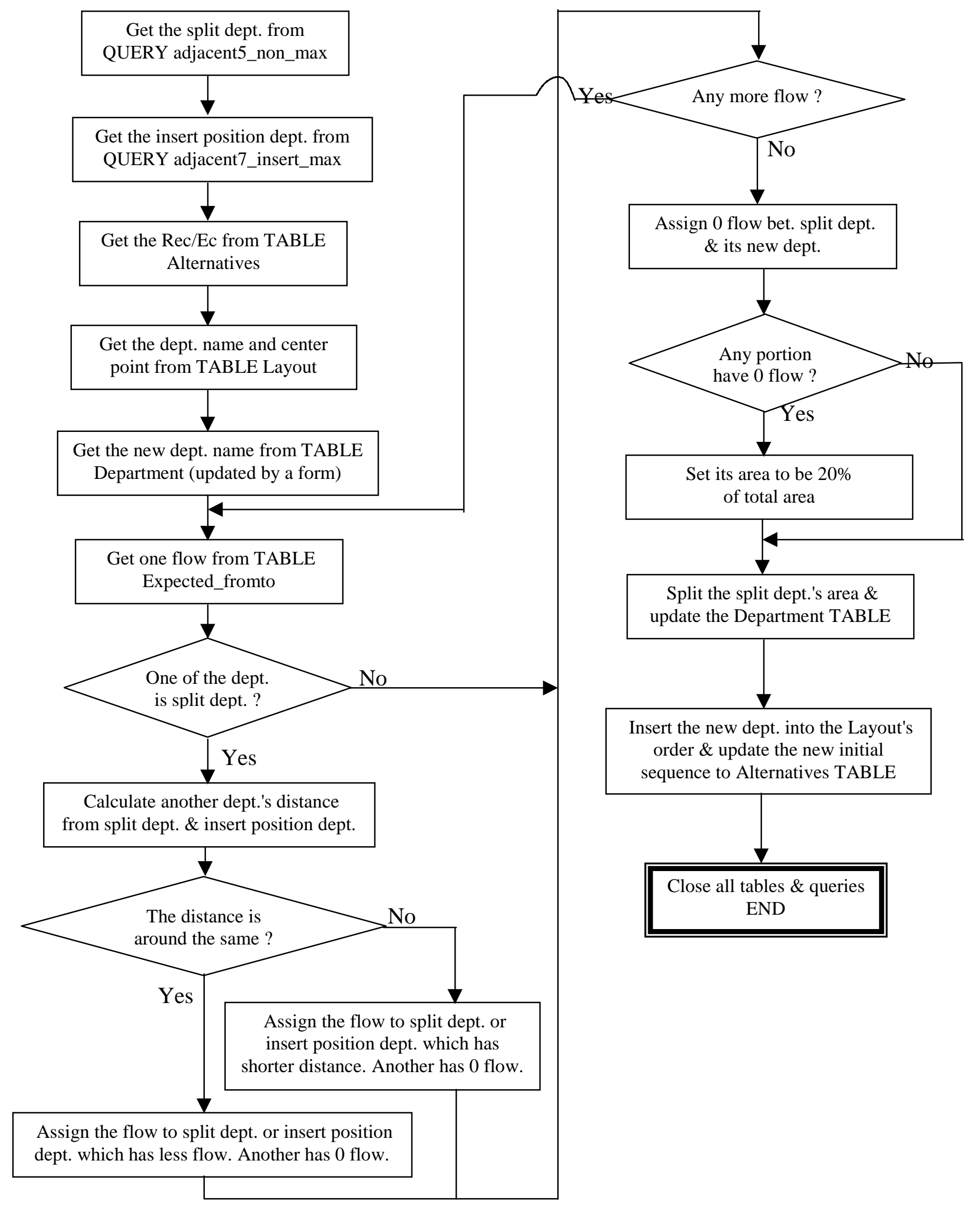

Figure 4-7 Program Flow of Module Split_dept 
pair exchange method again to check for possible further improvement. The procedure can be repeated until a satisfying result is reached. Figure 4-7 shows the program flow of the module Split_dept.

\subsection{Reschedule_flow Module}

This module is related to the Flexible Layout Design module. The Flexible Layout Design module uses the given material flows to create a layout. The purpose is to minimize the total material handling cost. The result is better than most other layout design methods. Chapter 5 will show the comparisons. However, it is a heuristic algorithm. It doesn't guarantee the optimal solution. Some improvements can be made here. One way is to do the scheduling again. Within the Flexible Layout Design Algorithm the schedule of forecasting material flows is integrated to get a better result. Here the future known material flows are rescheduled according to the final layout so that the total material handling cost can be reduced further.

In this research the material flow is considered to be uncertain. The layout designed by the method presented in this dissertation should be flexible regarding the change of material flow in the future. This module helps to realize that. If the future material flows turn out to be different from any possible results of forecasting or other methods, the layout created by the Flexible Layout Design Algorithm can shorten the distances between the departments and reduce the total material handling cost by rescheduling the material flows using this module. This makes the layout efficient and flexible. 
The Reschedule_flow module only makes sense after the Flexible Layout Design Algorithm is used. The Flexible Layout Design Algorithm provides the opportunity to reschedule. For layouts created by other methods, this module still works but the result is the same as before. There is no rescheduling. The Flexible Layout Design Algorithm makes the rescheduling possible.

Figure 4-8 shows the information relationships of this module. According to department positions in the layout from the table Layout and department split states from the table Department, the module Reschedule_flow checks two departments for each flow from the table Expected_fromto. If neither of these two departments is split, then nothing can be done. If one or both of them are split, then each combination of split portions including the original one is checked to see which pair has the shortest distance. Then the module Reschedule_flow reschedules the flow to these two departments. The table Expected_fromto is updated according to this change. All material flow will be checked.

Figure 4-9 shows the program flow of the module Reschedule_flow.

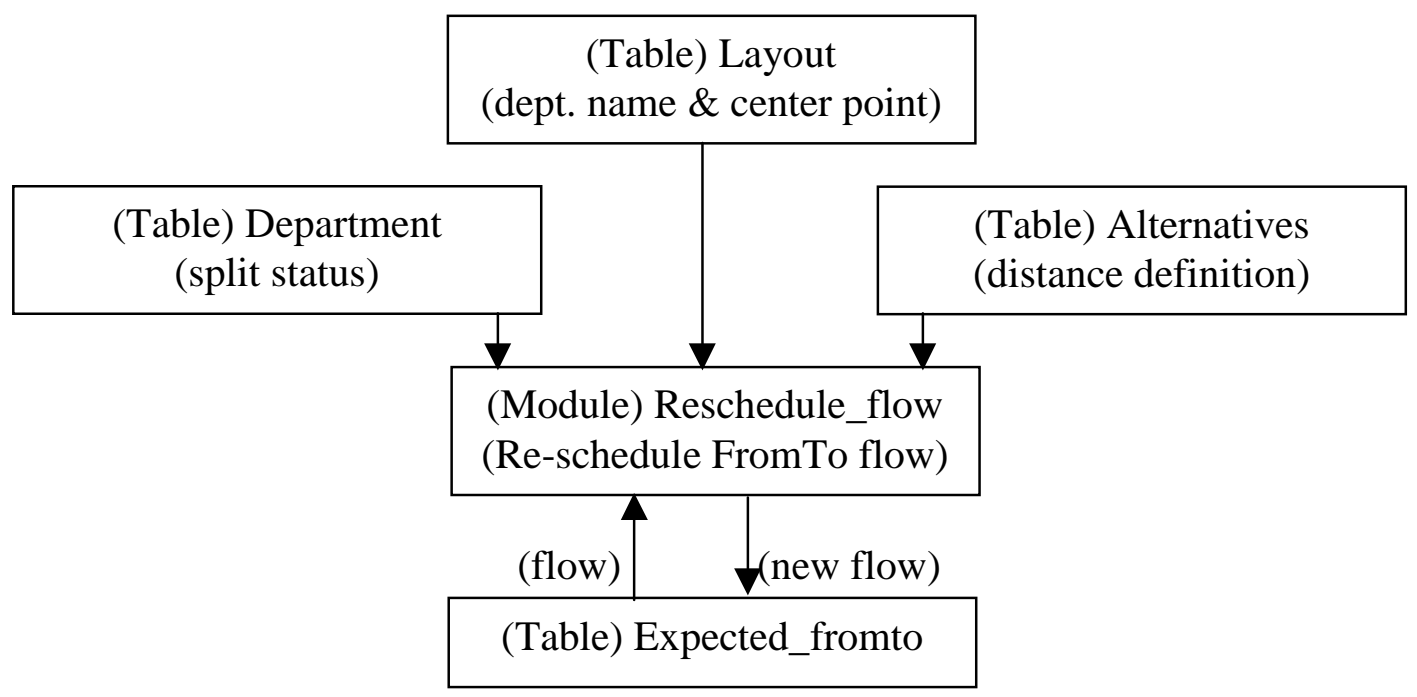

Figure 4-8 Reschedule Module Relationships 


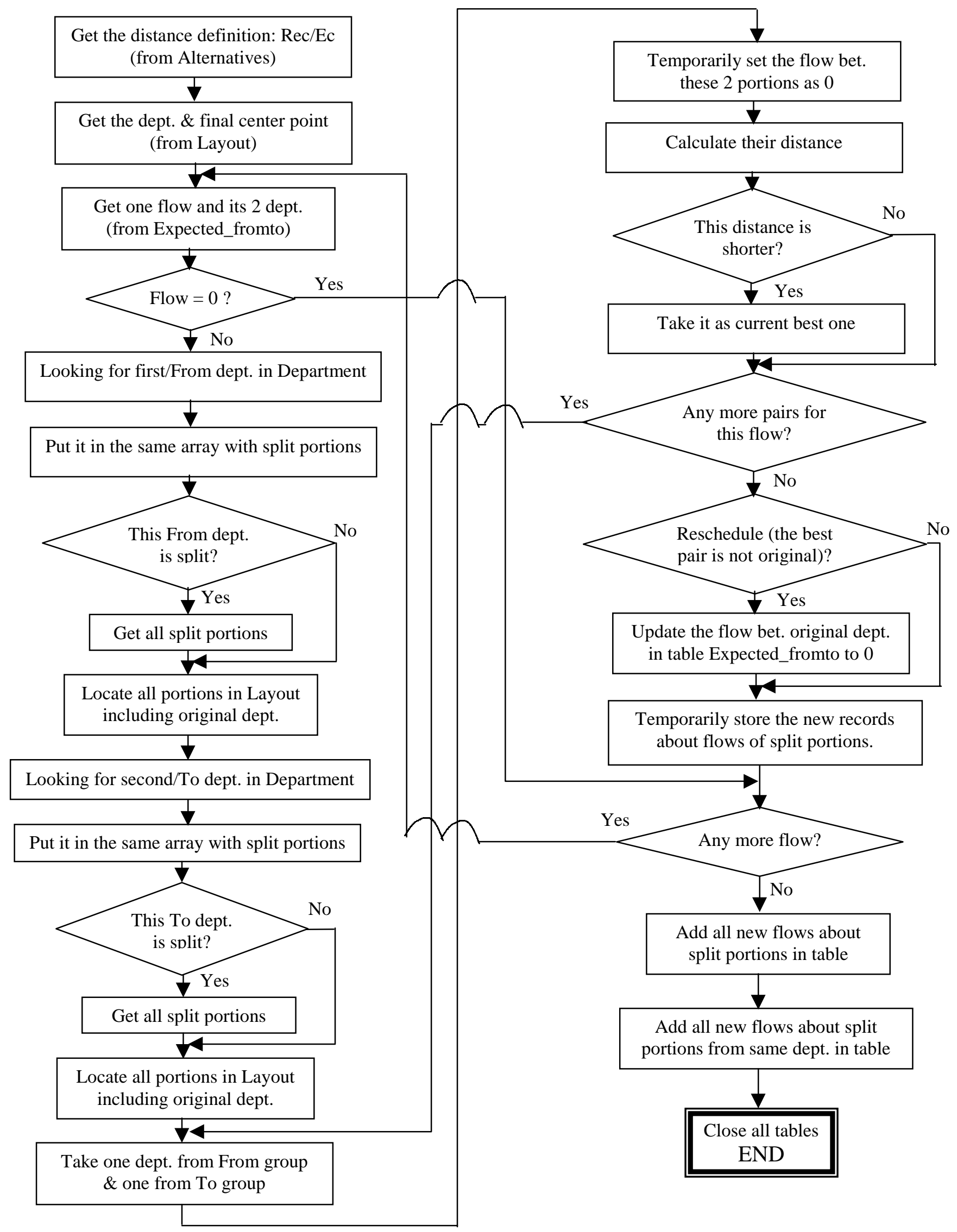

Figure 4-9 Program Flow of Module Reschedule_flow 


\subsection{Evaluation Module}

\subsubsection{OnlyPlaceLayout Module}

The Evaluation module is a relatively independent module. It can evaluate any other layouts, not necessarily from the pair exchange method or flexible layout design method. In order to realize this function, another module named the OnlyPlaceLayout was developed. The module OnlyPlaceLayout is a very simple evaluation tool. For any layout, if the area of departments and the order of departments are given, then the position of each department can be determined and the total material handling cost is calculated. The total material handling cost then can be used as a simple criterion for evaluation. The position of each department obtained here will be used for output of any layout's drawing and the evaluation module for more in-depth evaluating.

Figure 4-10 shows the OnlyPlaceLayout module relationships. Department area and order in the layout are used for determining positions of departments. Material flow is used for calculating the material handling cost.

Figure 4-11 shows the program flow of the module OnlyPlaceLayout. 


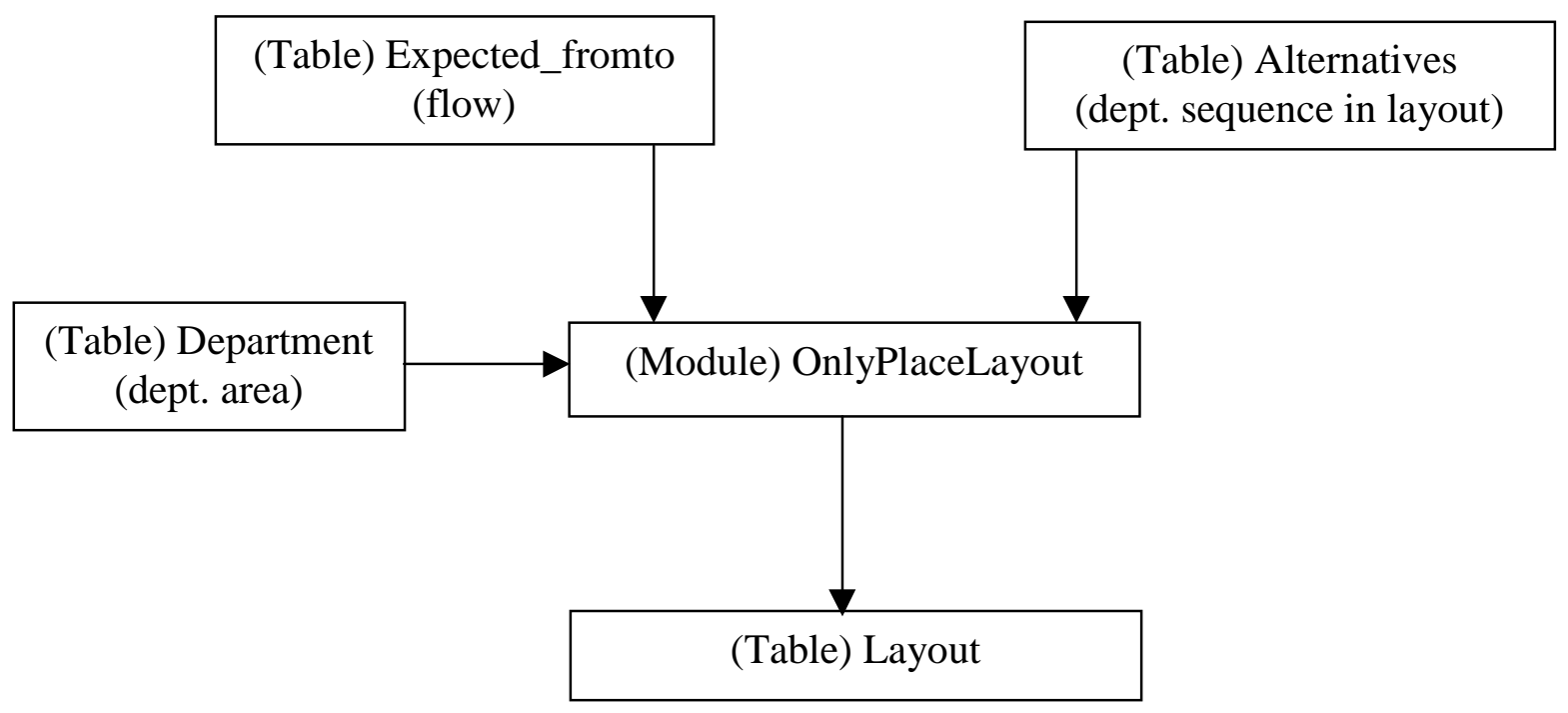

Figure 4-10 OnlyPlaceLayout Module Relationship

\subsubsection{SequenceToFlow Module}

Here the sequence of operations instead of from-to flow is used for evaluation. They are consistent. The sequence of operations can be converted to from-to flow. The module SequenceToFlow realizes this function. Figure 4-12 shows the relationships of the module SequenceToFlow. It separates the sequence of operations and combines the same flows between two departments. The result is the quantity of material flow, which is stored in the table Quantity_per_month.

Figure 4-13 shows the program flow of the module SequenceToFlow. 


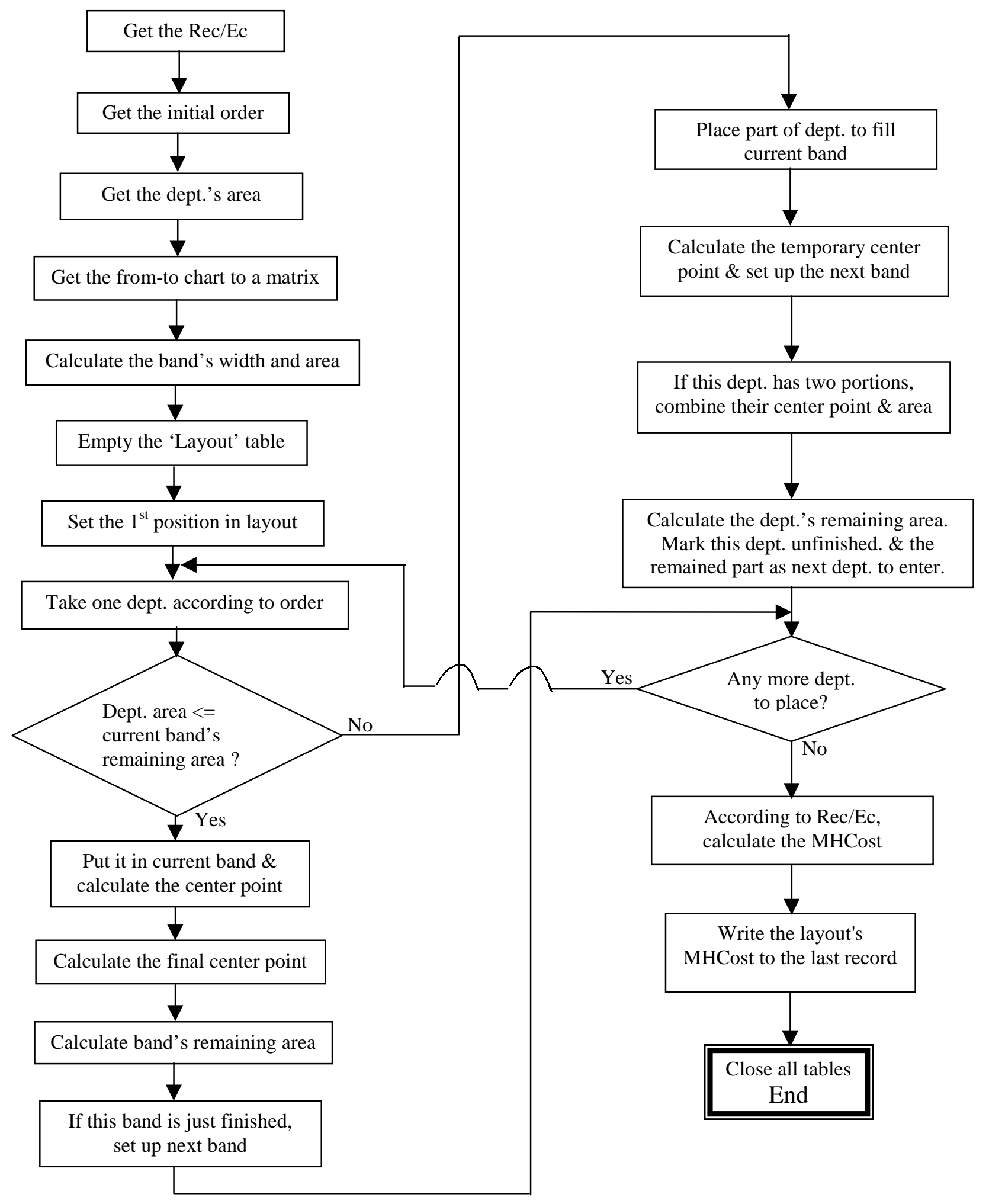

Figure 4-11 The Program Flow of Module OnlyPlaceLayout 


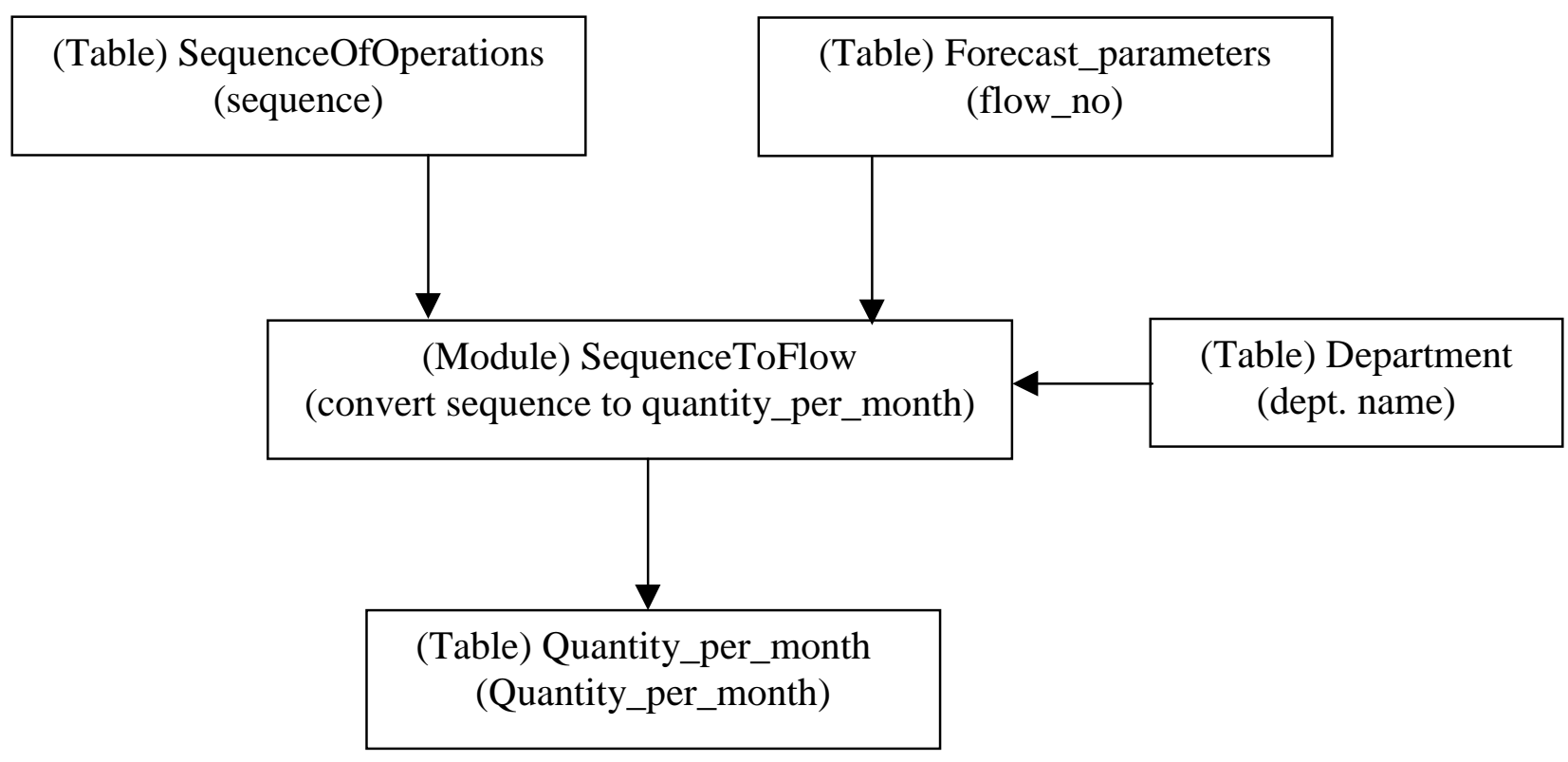

Figure 4-12 SequenceToFlow Module Relationships

\subsubsection{Evaluation Module}

Figure 4-14 shows the Evaluation module relationships. Department positions from the table Layout are used for distance calculation. Parts' quantity distribution and sequence of operations are from the table SequenceOfOperations. Other data are from the tables Alternatives, MHS_material and MHS. The result, MHCost distribution, is stored in the table Evaluation.

Figure 4-15 shows the program flow of the module Evaluation. 


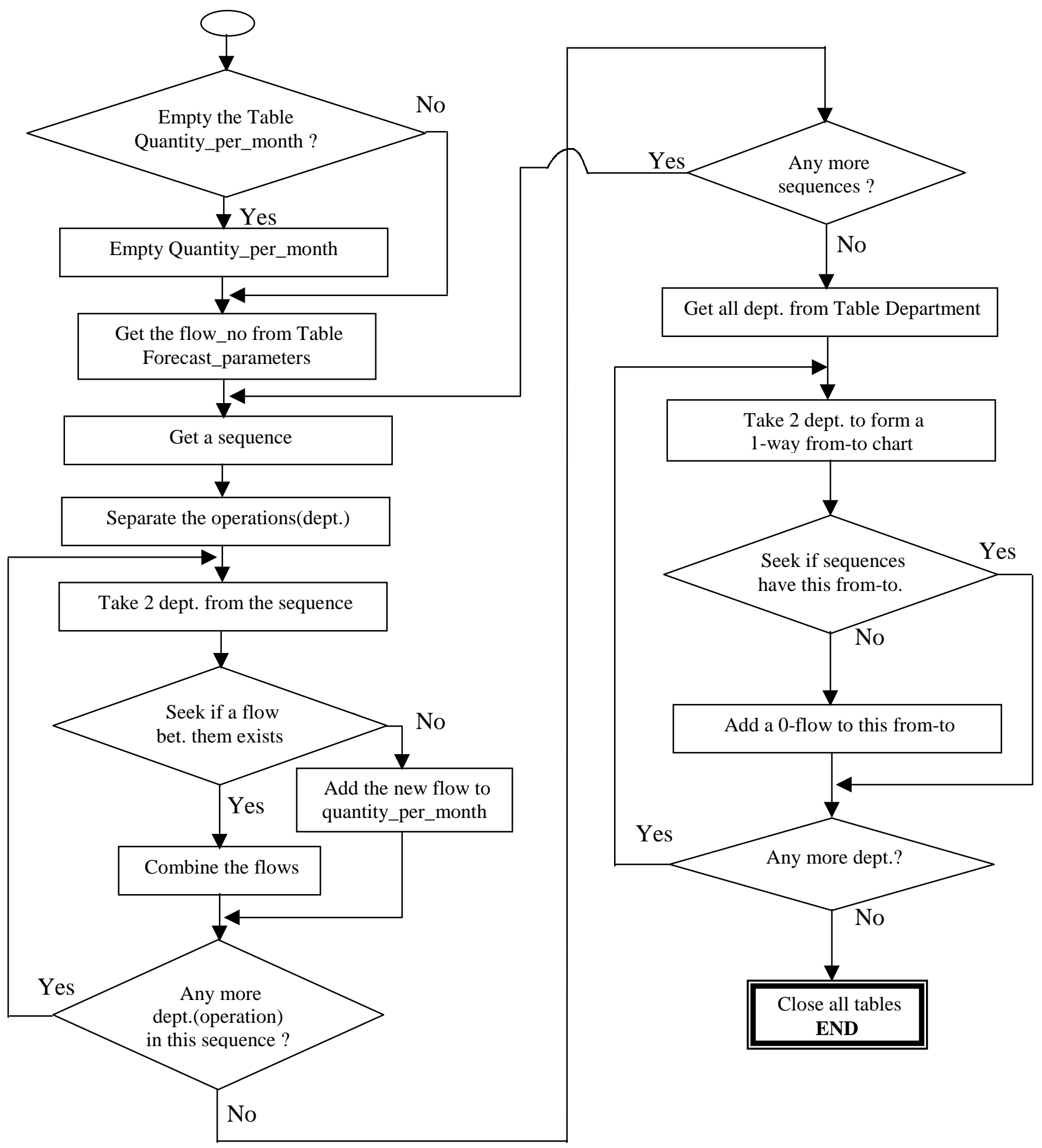

Figure 4-13 Program Flow of Module SequenceToFlow 


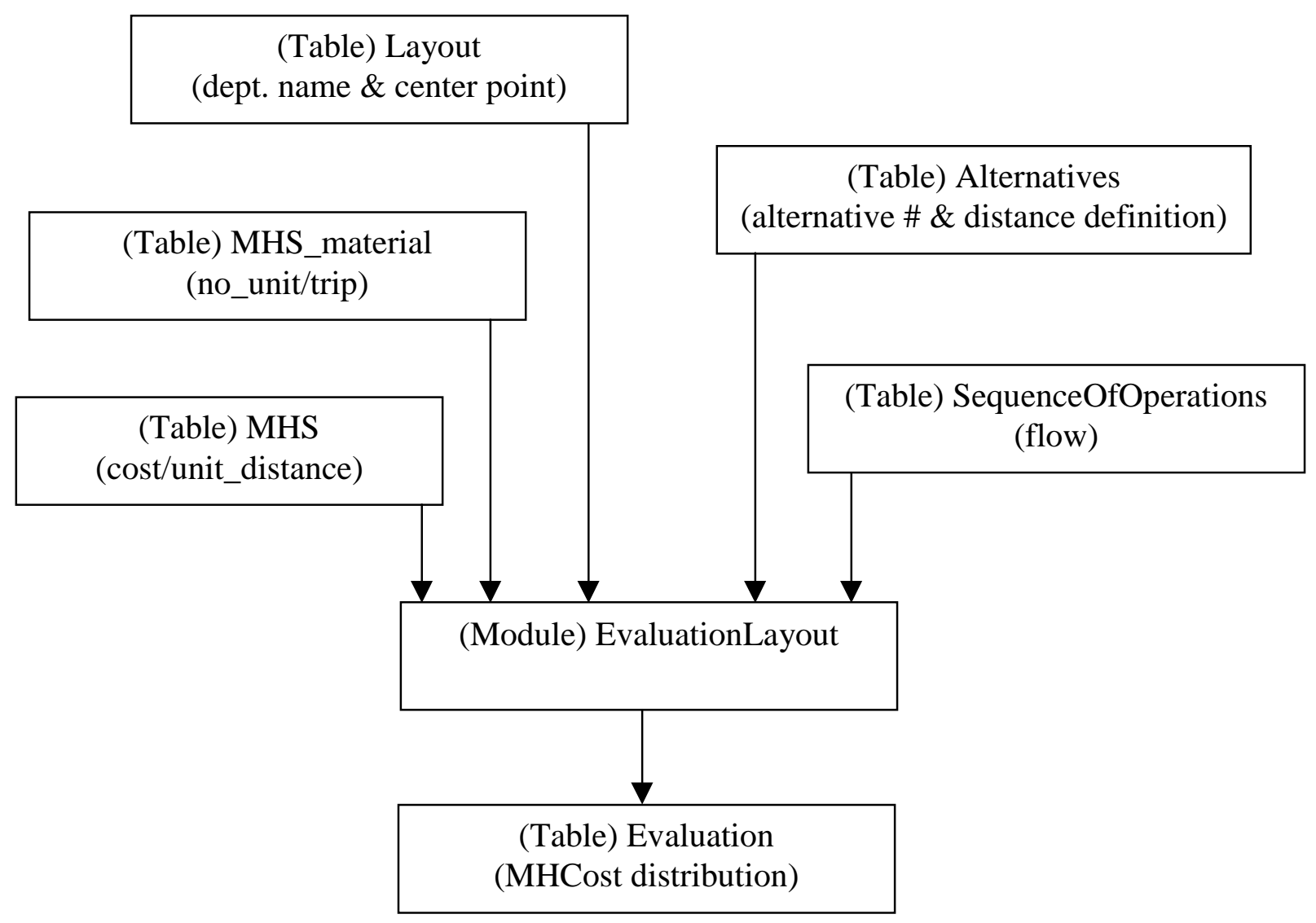

Figure 4-14 Evaluation Module Relationships

\subsubsection{Resequence Module}

If the flexible layout design method is used, some departments may be split. In this case, the parts should be re-scheduled to have a better sequence of operations, i.e. lower total material handling cost. The module Resequence was developed to realize this function. Figure 4-16 shows the Resequence module relationships. For each sequence of operations, every department from the department table is checked to see if it is split. If 


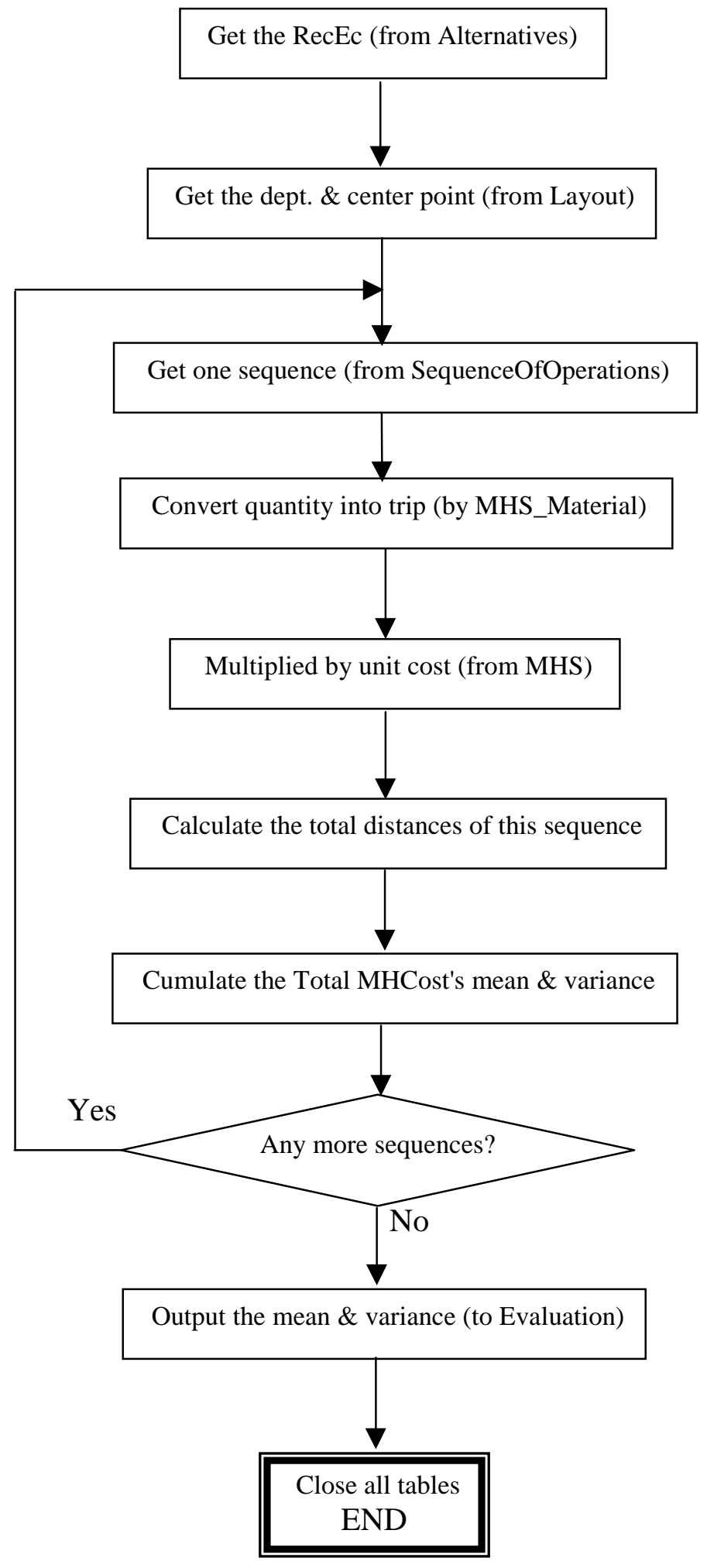

Figure 4-15 Program Flow of Module Evaluation 


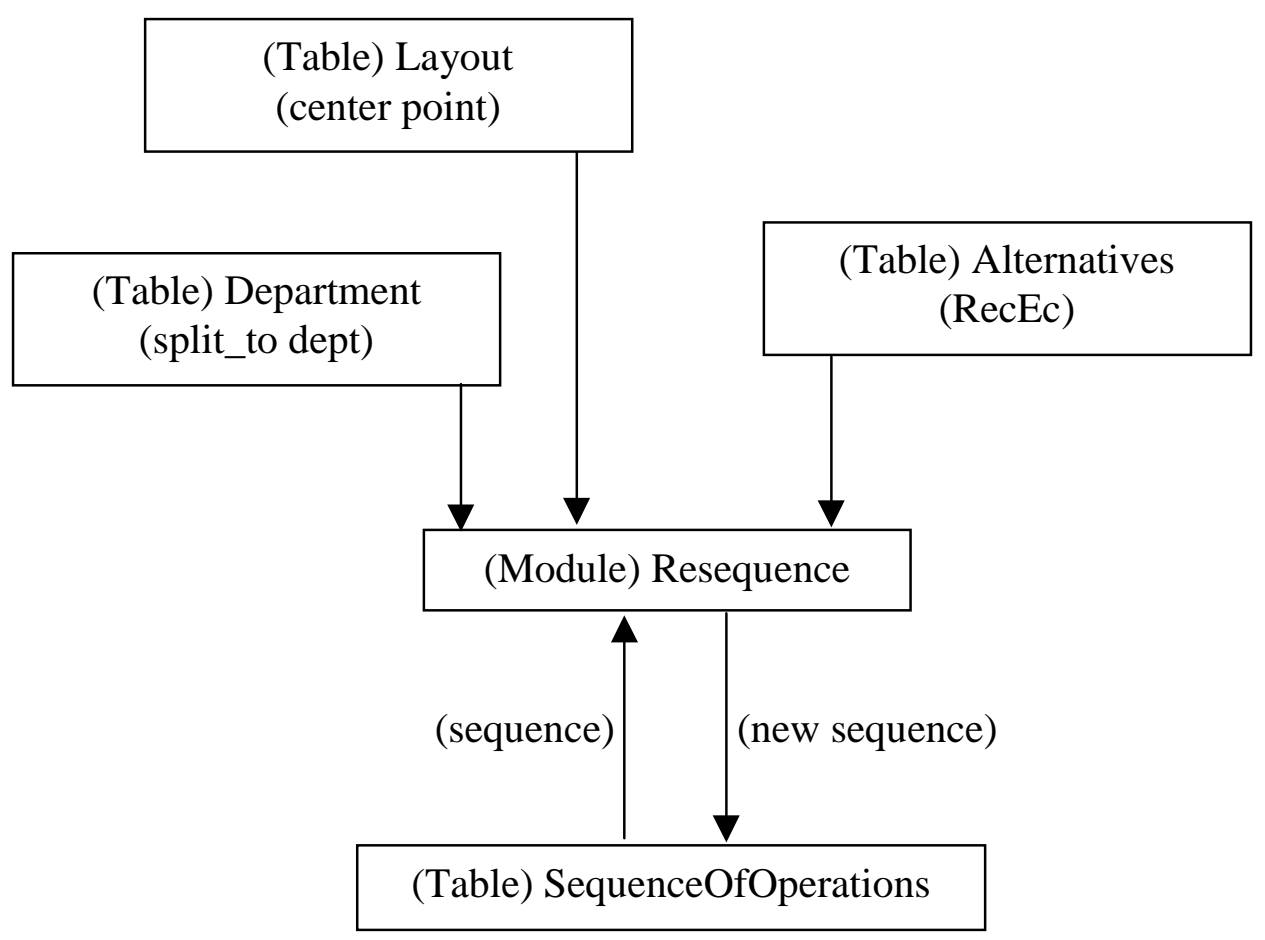

Figure 4-16 Resequence Module Relationships

it is split, then its new portions in the layout from the Layout table are checked to see which portion is selected to have less material handling cost. That means re-sequencing the part. The new sequence is still stored in table the SequenceOfOperations.

Figure 4-17 shows the program flow of the module Resequence. 


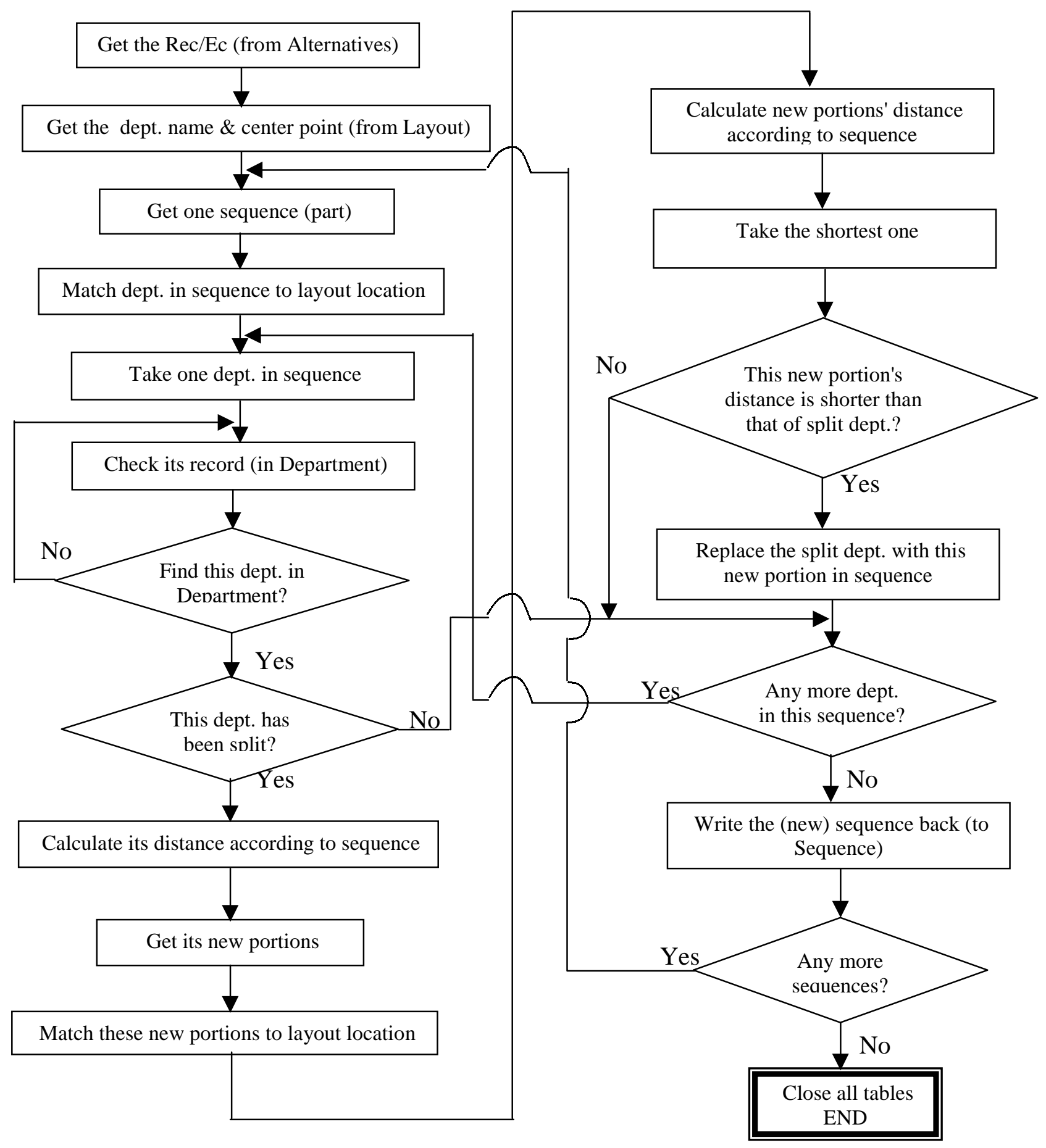

Figure 4-17 Program Flow of Module Resequence 


\subsection{Drawing Module}

The Drawing module draws the layout in two-dimensional space. Figure 4-18 shows the Drawing module relationships. The Drawing module gets information from the tables Layout, Alternatives and Department. Then it draws the position of each department to the report of Microsoft Access. Different colors are used to identify the departments. The total material handling cost of this layout and the department split status are also displayed in the report.

Figure 4-19 shows the program flow of the Drawing module.

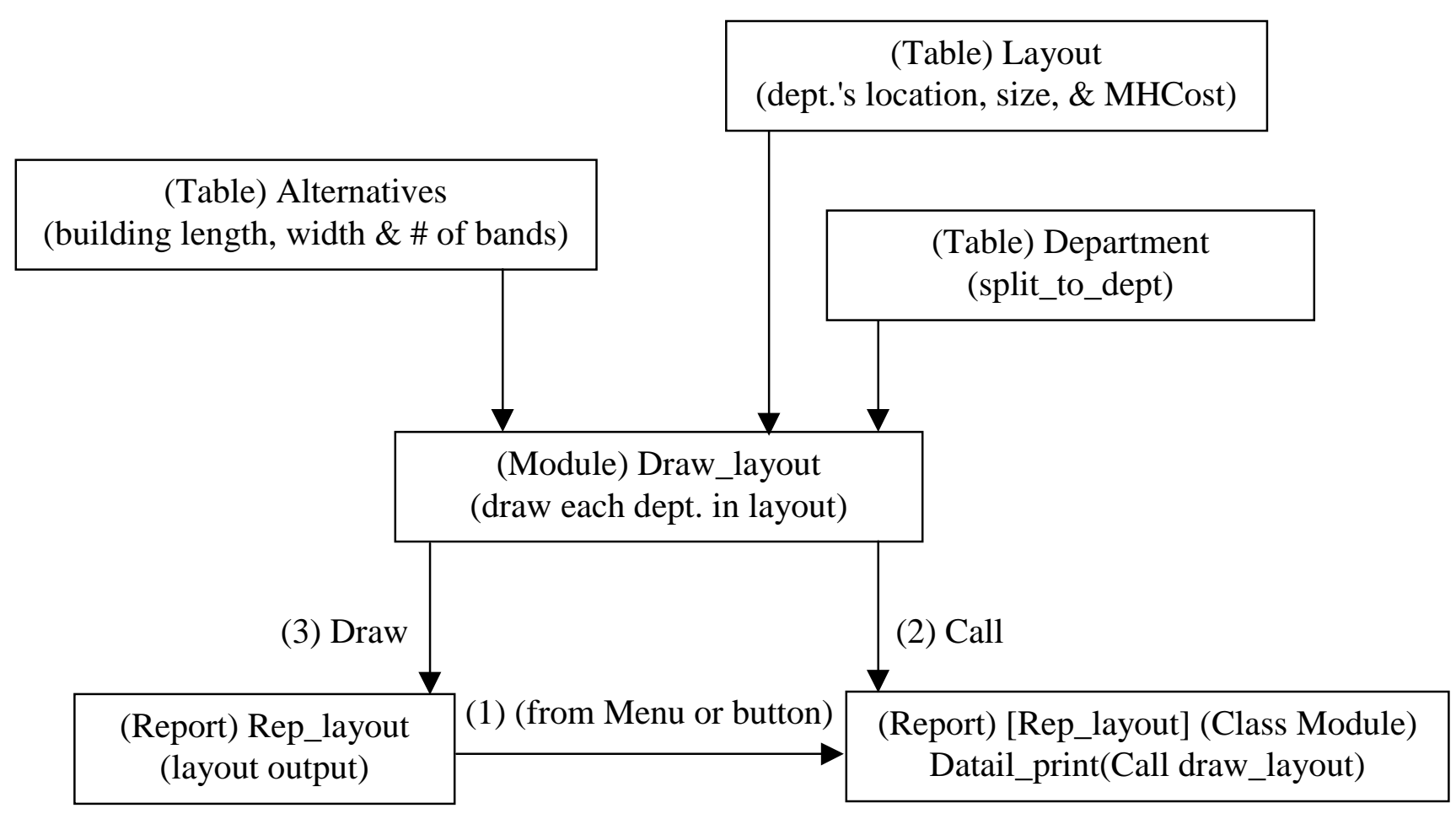

Figure 4-18 Module Drawing_layout Relationships 


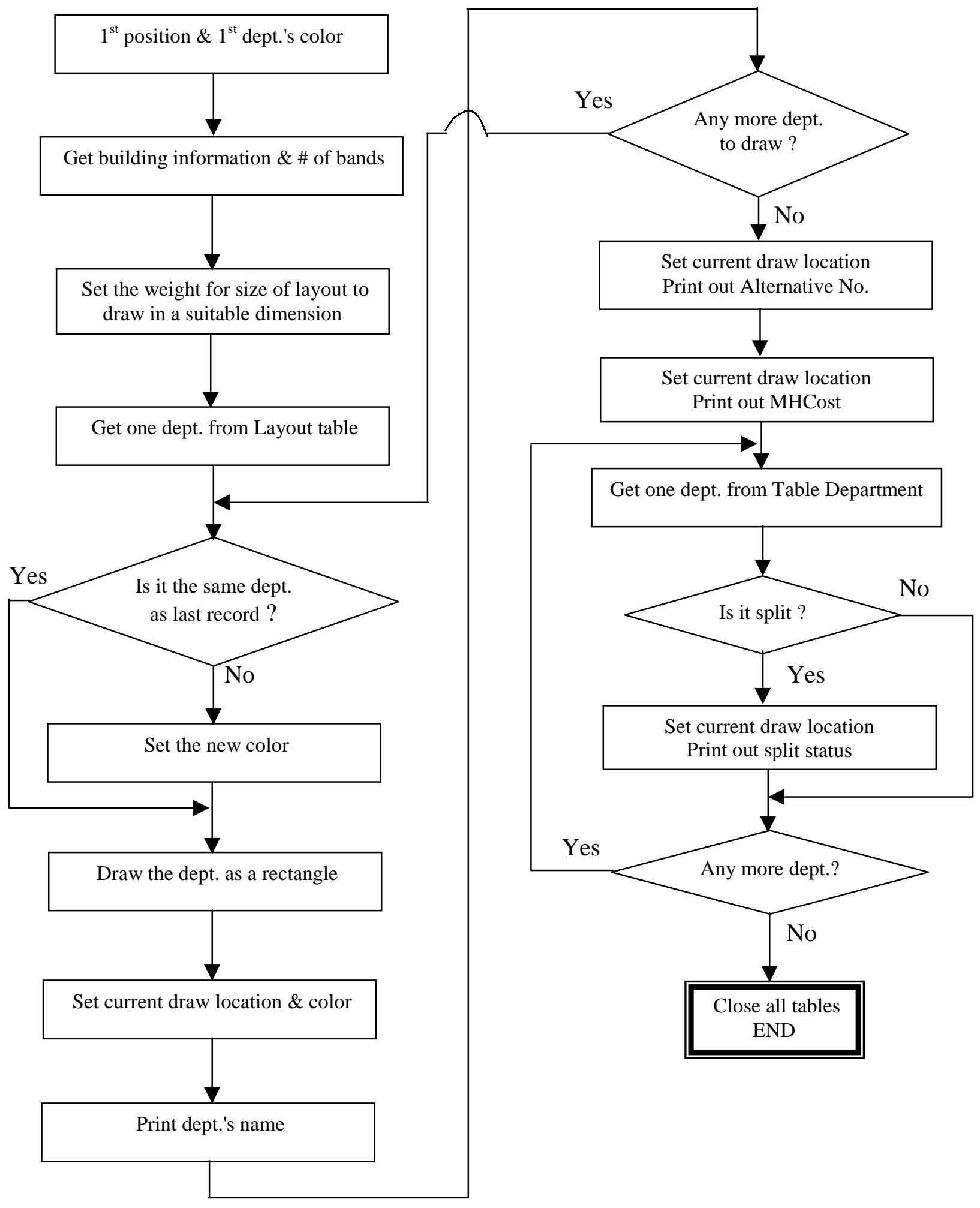

Figure 4-19 Program Flow of Module Drawing 


\subsection{System Integration}

There are ten sub-systems. They all have their own functions, but they interact with each other and share much information. These sub-systems do not necessarily run strictly one after another so it is very important to integrate all these sub-systems.

These sub-systems do not call each other. There is not any argument passing from one sub-system to another. Any changes of one sub-system have nothing to do with other sub-systems. Even removing or adding any sub-systems does not affect other subsystems. All sub-systems use a Microsoft Access database for information sharing. All input information given by users is stored in the database's tables. Each sub-system gets the input information from the tables. The results put out from the sub-systems are also stored in the tables. The results from one sub-system may be the input information to other sub-systems. All sub-systems are integrated by a database. The overall program structure in Figure 4-1 also shows this integration.

Table Layout stores the most important shared information. It has the position of each department in the layout. The results from the pair exchange method by the module PairExchange or from the simple evaluation method by the module OnlyPlaceLayout are stored here. The Drawing module uses the data in the table Layout to draw a figure of the building and departments. The Flexible layout design module uses the data in the table Layout as initial layout information and improves it. The Reschedule flow module uses data in the table Layout to calculate the distances among departments. The Evaluation module uses the data in the table Layout to calculate the total material handling cost's 
distribution. The Resequence module uses the data in the table Layout to re-schedule the parts' sequence of operations. It is clearly defined that only the modules PairExchange and OnlyPlaceLayout can edit the table Layout. The modules Split_dept, EvaluationLayout, Resequence and Draw_layout can only read the table Layout. All these sub-systems react easily by sharing the information in the table Layout.

The table Expected_fromto is another important entity used for system integration. Results from the Forecasting module are converted and stored in this table. Users have the option to input the information stored in this table without using the Forecasting module. The pair exchange layout design algorithm uses this information as the from-to chart to create a layout and calculates the total material handling cost. The module OnlyPlaceLayout also uses this information to calculate the total material handling cost. The Flexible layout design module and the Reschedule flow module rearrange the fromto material flow stored in table Expected_fromto to reduce the total material handling cost.

The table Department also stores information shared by several sub-systems. At the beginning, users should input the department information such as name, area, etc. stored in this table. The module Split_dept changes the information in the table Department after a department is split. The modules PairExchange and OnlyPlaceLayout use the departments' name and area stored in the table Department to place the departments in the layout and calculate the total material handling cost. The module Reschedule Flow checks the split states of departments in the table Department. The module Resequence uses the split status of departments stored in the table Department to reschedule the 
sequences of operations of parts. The module SequenceToFlow also uses the information in the table Department to convert the sequences of operations of parts to a material flow from-to chart.

\subsection{Conclusion}

In this chapter the overall program structure and sub-system structures are presented. For each sub-system, usually two aspects are discussed. One is the sub-system's relationship, which is the interface of this sub-system. All sub-systems do not interact with each other directly; they are relatively independent. It is easy to develop and maintain. The data entities are used to integrate all these sub-systems and ensure they work together properly. The other one is the sub-system's program flow. It shows the functions of this sub-system. Appendix I lists all the data in data entities. 


\section{SYSTEM IMPLEMENTATION AND VALIDATION}

\subsection{System Implementation}

The system is implemented by using Microsoft Access and Visual Basic by Application. All information needed for layout design and the result from the layout design algorithm are stored in Microsoft Access's tables. Microsoft Access's query is used for simple calculation. Visual Basic by Application is used to implement the algorithms. Microsoft Access's report is used to output the layouts to the screen or to the printer.

\subsection{Deterministic Material Flow Case Study}

The case study presented here is taken from J.A. Tompkins, J.A. White, et al, Facilities Planning, $2^{\text {nd }}$ edition, 1996. pp324-325, problem 7.23. There are ten departments. Their areas are different and given. The material flow is assumed to be deterministic and known. The change in material flow is not taken into account. The problem is to arrange these ten departments into a rectangular plant building to minimize the total material handling cost.

Table 5-1 shows the material flows of the departments. 


\section{Table 5-1 Material Flows Chart}

\begin{tabular}{||l|r|r|r|r|r|r|r|r|r|r|r||}
\hline Dept & Area & A & B & C & D & E & F & G & H & I & J \\
\hline A & 400 & - & 0 & 12 & 0 & 132 & 16 & 0 & 220 & 20 & 24 \\
\hline B & 1000 & 0 & - & 176 & 0 & 216 & 0 & 144 & 128 & 0 & 0 \\
\hline C & 2600 & 0 & 0 & - & 0 & 0 & 184 & 0 & 0 & 28 & 0 \\
\hline D & 400 & 212 & 136 & 240 & - & 36 & 0 & 236 & 0 & 164 & 0 \\
\hline E & 2400 & 0 & 0 & 140 & 0 & - & 0 & 192 & 0 & 0 & 160 \\
\hline F & 1000 & 0 & 180 & 0 & 188 & 108 & - & 248 & 228 & 0 & 0 \\
\hline G & 3600 & 172 & 0 & 156 & 0 & 0 & 0 & - & 112 & 224 & 152 \\
\hline H & 1200 & 0 & 0 & 32 & 40 & 204 & 0 & 0 & - & 0 & 0 \\
\hline I & 400 & 0 & 168 & 0 & 0 & 104 & 156 & 0 & 148 & - & 200 \\
\hline J & 2400 & 0 & 124 & 196 & 120 & 0 & 116 & 0 & 108 & 0 & - \\
\hline
\end{tabular}

It is assumed unit cost $c_{i j}=\$ 1 /$ unit load/unit distance for any department $\mathrm{i}, \mathrm{j}$ in this problem. The input layout is A-B-C-D-E-F-G-H-I-J. This order expression is the same as the one used by CRAFT. The number of bays is set to three. Travel distances are rectilinear. The resulting layout from CRAFT is H-A-I-D-F-J-G-E-B-C. The total material handling cost is $\$ 445,476.80$. The resulting layout from the pair exchange layout design algorithm presented in this dissertation is E-B-C-F-D-I-A-H-J-G. The total material handling cost is $\$ 406,985.10$.

After the flexible layout design algorithm is used in this case, department $\mathrm{J}$ is split to departments $\mathrm{J}$ and $\mathrm{X}$. The material flows are rescheduled as shown in Table 5-2. 
Table 5-2 Material Flows of Departments J and X

\begin{tabular}{||c|r|r|r|r|r|r|r|r|r|r|r||}
\hline \hline DEPT & A & B & C & D & E & F & G & H & I & J & X \\
\hline J & 0 & 124 & 0 & 0 & 160 & 0 & 0 & 108 & 0 & - & 0 \\
\hline X & 24 & 0 & 196 & 120 & 0 & 116 & 152 & 0 & 200 & 0 & - \\
\hline
\end{tabular}

The resulting layout from this algorithm is E-C-X-D-I-F-B-J-H-A-G. The total material handling cost is $\$ 390,113.9$. The layout is shown in Figure 5-1.

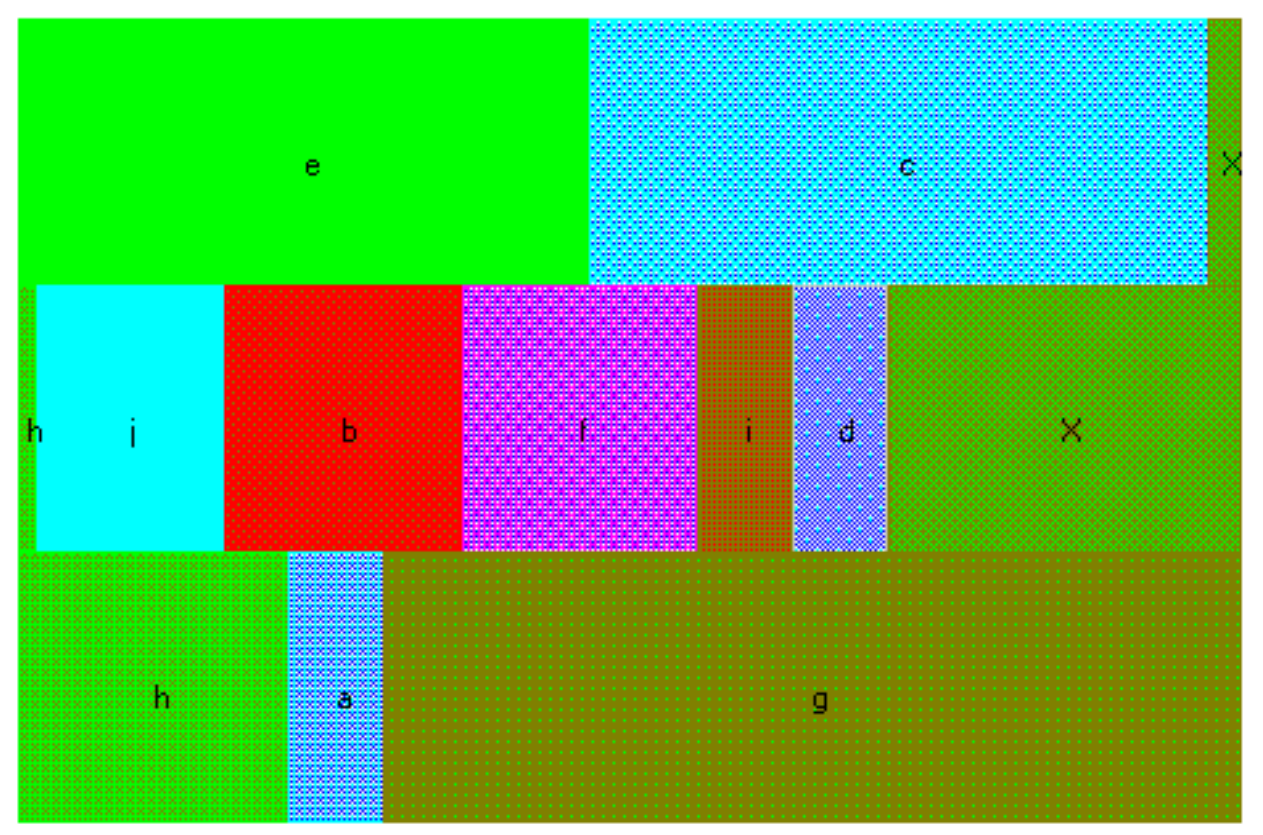

Figure 5-1 Layout After Department J Is Split

Compared to CRAFT, the cost saving is $445,476.80-390,113.90=\$ 55,362.90$, more than a $12 \%$ cost reduction. 
A better result can be obtained by using the Reschedule_flow module to reschedule the material flow. In this case, the total material handling cost is $\$ 387,402.80$. Compared to CRAFT, the cost saving is $445,476.80-387,402.80=\$ 58,074.00$, more than a $13 \%$ cost reduction.

The flexible layout design algorithm can be used for multiple splits. If the splits continued, department $\mathrm{I}$ is split to departments $\mathrm{I}$ and $\mathrm{Y}$. The material flows are rescheduled as shown in Table 5-3. The resulting layout from this algorithm is E-C-X-YD-F-I-B-J-H-A-G. The total material handling cost is $\$ 382,147.70$. The layout is shown in Figure 5-2.

Table 5-3 Material Flows of Department I and Y

\begin{tabular}{||r|r|r|r|r|r|r|r|r|r|r|r|r||}
\hline \hline DEPT & A & B & C & D & E & F & G & H & I & J & X & Y \\
\hline I & 20 & 0 & 28 & 160 & 104 & 156 & 0 & 148 & - & 0 & 0 & 0 \\
\hline Y & 0 & 168 & 0 & 0 & 0 & 0 & 224 & 0 & 0 & 0 & 200 & - \\
\hline
\end{tabular}

After rescheduling, the total material handling cost is $\$ 366,118.40$. Compared to CRAFT, the cost saving is $445,476.80-366,118.40=\$ 79,358.40$, more than a $17 \%$ cost reduction.

If the procedure continues, next department $\mathrm{D}$ is split to departments $\mathrm{D}$ and $\mathrm{Z}$. The material flows are rescheduled as showed in Table 5-4. The resulting layout from this algorithm is E-C-X-D-Y-F-I-B-J-H-A-Z-G. The total material handling cost is $\$ 363,860.30$. The layout is shown in Figure 5-3. 


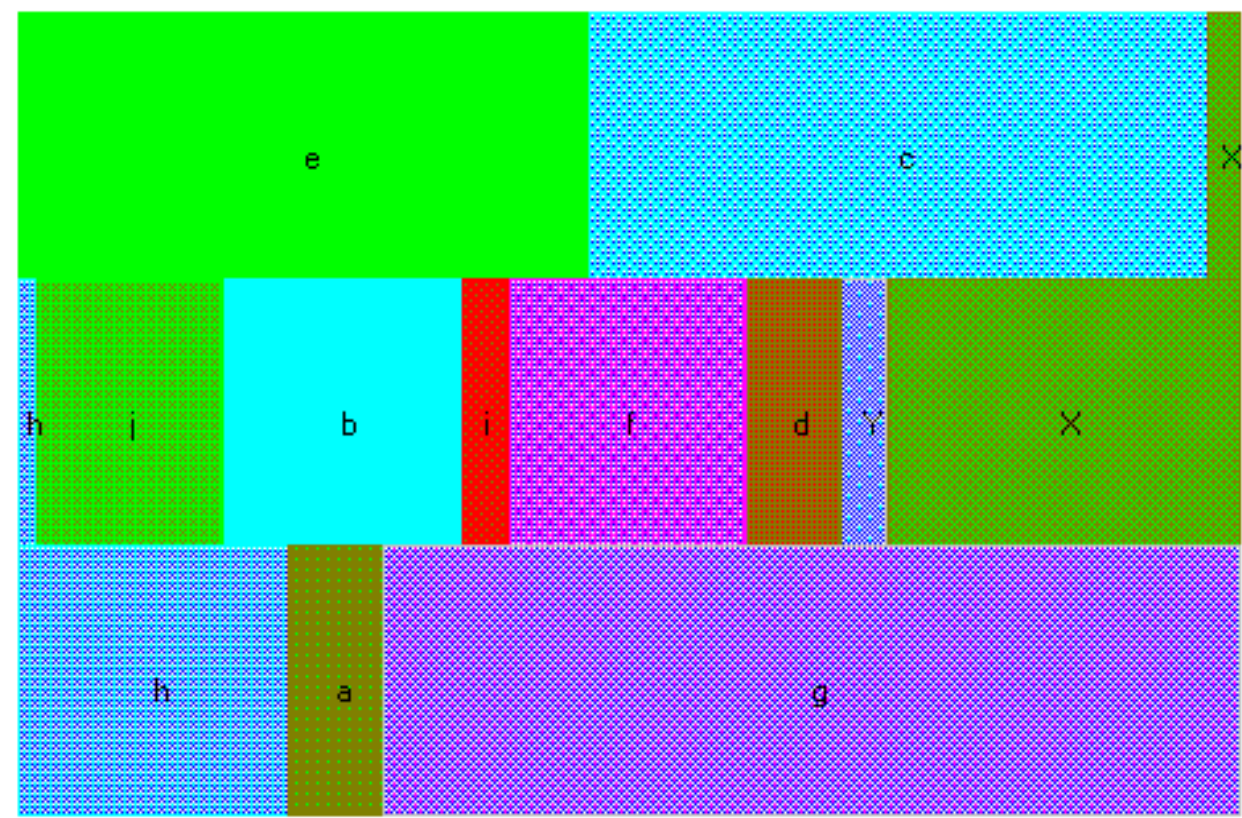

Figure 5-2 Layout After Department L Is Split

Table 5-4 Material Flows of Departments $D$ and $Z$

\begin{tabular}{|c|r|r|r|r|r|r|r|r|r|r|r|r|r||}
\hline DEPT & A & B & C & D & E & F & G & H & I & J & X & Y & Z \\
\hline D & 0 & 136 & 240 & - & 0 & 188 & 0 & 0 & 164 & 0 & 120 & 0 & 0 \\
\hline Z & 212 & 0 & 0 & 0 & 36 & 0 & 236 & 40 & 0 & 0 & 0 & 0 & - \\
\hline
\end{tabular}




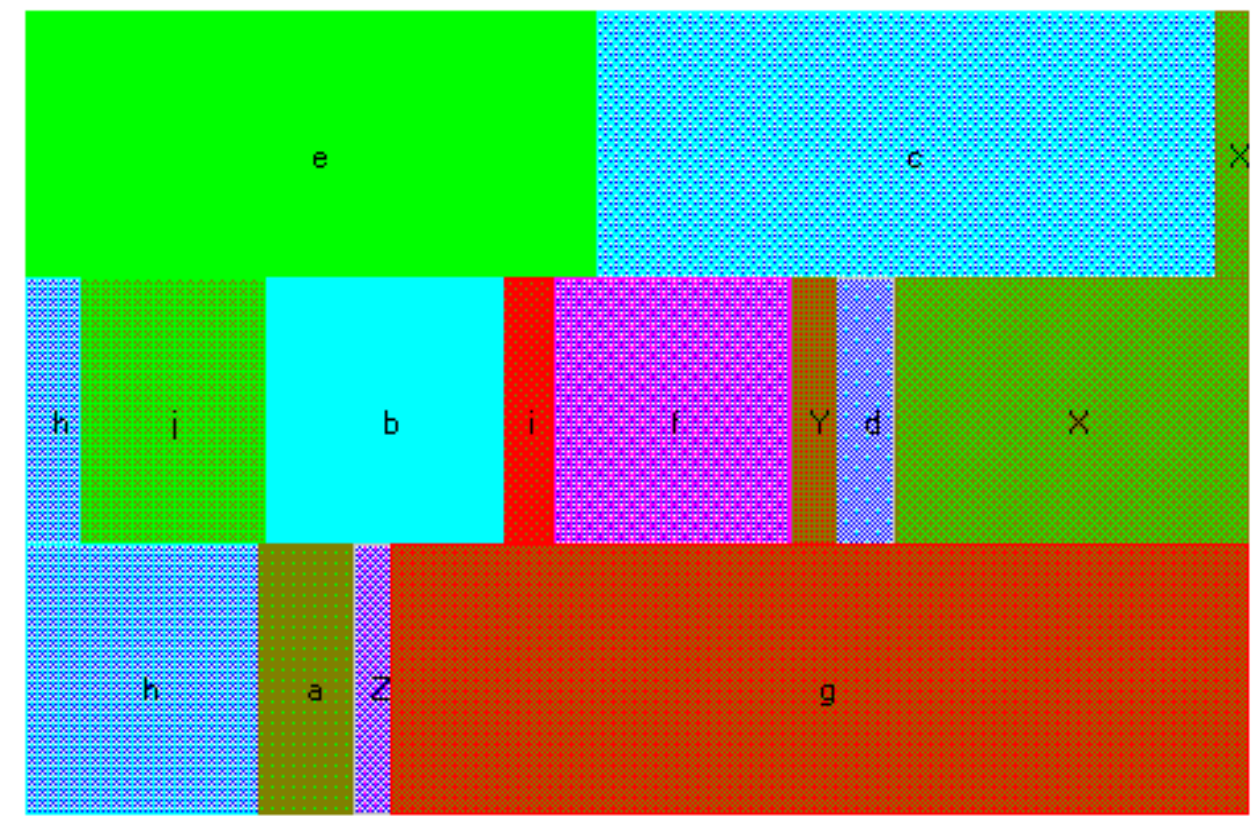

Figure 5-3 Layout After Department D Is Split

After rescheduling, the total material handling cost is $\$ 341,290.10$. Compared to CRAFT, the cost saving is $445,476.80-341,290.10=\$ 104,186.70$, more than a $23 \%$ cost reduction.

\subsection{Validation of Literature Data}

This case is taken from M.J. Rosenblatt, D.H. Kropp, The Single Period Stochastic Plant Layout Problem, IIE Transaction, 1992. v24, n2, pp169-176. This case was also used in M.J. Rosenblatt, The Dynamics of Plant Layout, Management Science, 32, 1, 1986. pp76-86.

There are six total departments. Their areas are given. The material flow is assumed to be stochastic and uncertain. Under this condition, five possible flow sets 
among the departments are given. The data are shown in Table 5-5. The problem is to arrange these six departments into a rectangular plant building to minimize the total material handling cost.

Table 5-5 Five Possible Material Flow Charts

\begin{tabular}{||c|r|r|r|r|r|r|r||}
\hline Dept. & Area & 1 & 2 & 3 & 4 & 5 & 6 \\
\hline 1 & 1 & - & 63 & 605 & 551 & 116 & 136 \\
\hline 2 & 1 & 63 & - & 635 & 941 & 50 & 191 \\
\hline 3 & 1 & 104 & 71 & - & 569 & 136 & 55 \\
\hline 4 & 1 & 65 & 193 & 622 & - & 77 & 90 \\
\hline 5 & 1 & 162 & 174 & 607 & 591 & - & 179 \\
\hline 6 & 1 & 156 & 13 & 667 & 611 & 175 & - \\
\hline
\end{tabular}

\begin{tabular}{||c|c|r|r|r|r|r|r||}
\hline Dept. & Area & 1 & 2 & 3 & 4 & 5 & 6 \\
\hline 1 & 1 & - & 175 & 804 & 904 & 56 & 176 \\
\hline 2 & 1 & 63 & - & 743 & 936 & 45 & 177 \\
\hline 3 & 1 & 168 & 85 & - & 918 & 138 & 134 \\
\hline 4 & 1 & 51 & 94 & 962 & - & 173 & 39 \\
\hline 5 & 1 & 97 & 104 & 730 & 634 & - & 144 \\
\hline 6 & 1 & 95 & 115 & 983 & 597 & 24 & - \\
\hline
\end{tabular}




\begin{tabular}{||c|r|r|r|r|r|r|r||}
\hline Dept. & Area & 1 & 2 & 3 & 4 & 5 & 6 \\
\hline 1 & 1 & - & 90 & 77 & 553 & 769 & 139 \\
\hline 2 & 1 & 168 & - & 114 & 653 & 525 & 185 \\
\hline 3 & 1 & 32 & 35 & - & 664 & 898 & 87 \\
\hline 4 & 1 & 27 & 166 & 42 & - & 960 & 179 \\
\hline 5 & 1 & 185 & 56 & 44 & 926 & - & 104 \\
\hline 6 & 1 & 72 & 128 & 173 & 634 & 687 & - \\
\hline
\end{tabular}

\begin{tabular}{||c|r|r|r|r|r|r|r||}
\hline Dept. & Area & 1 & 2 & 3 & 4 & 5 & 6 \\
\hline 1 & 1 & - & 112 & 15 & 199 & 665 & 649 \\
\hline 2 & 1 & 153 & - & 116 & 173 & 912 & 671 \\
\hline 3 & 1 & 10 & 28 & - & 182 & 855 & 542 \\
\hline 4 & 1 & 29 & 69 & 15 & - & 552 & 751 \\
\hline 5 & 1 & 198 & 71 & 42 & 24 & - & 758 \\
\hline 6 & 1 & 62 & 109 & 170 & 90 & 973 & - \\
\hline
\end{tabular}

\begin{tabular}{||c|r|r|r|r|r|r|r||}
\hline Dept. & Area & 1 & 2 & 3 & 4 & 5 & 6 \\
\hline 1 & 1 & - & 663 & 23 & 128 & 119 & 50 \\
\hline 2 & 1 & 820 & - & 5 & 98 & 141 & 66 \\
\hline 3 & 1 & 822 & 650 & - & 137 & 78 & 91 \\
\hline 4 & 1 & 826 & 570 & 149 & - & 93 & 151 \\
\hline 5 & 1 & 915 & 515 & 53 & 35 & - & 177 \\
\hline 6 & 1 & 614 & 729 & 178 & 10 & 99 & - \\
\hline
\end{tabular}




\subsubsection{Flexible Layout Design Method and Comparison to CRAFT Method}

A unit cost $c_{i j}=\$ 1 /$ unit load/unit distance for any department $\mathrm{i}, \mathrm{j}$ in this problem is assumed. The input layout is 1-2-3-4-5-6. This order expression is the same as the one used by CRAFT. The number of bays is set to two. Travel distances are rectilinear. In order to use the algorithms, expected flows are calculated and used as the material flow chart. The resulting layout from CRAFT is 3-4-2-1-5-6. The total material handling cost is $\$ 14,796.40$. The resulting layout from the pair exchange layout design algorithm presented in this dissertation is the same as CRAFT. The total material handling cost is also $\$ 14,796.40$.

After the flexible layout design algorithm is used in this case, department 3 is split to departments 3 and $\mathrm{X}$. The material flows are rescheduled as shown in Table 5-6.

Table 5-6 Material Flows of Departments 3 and X

\begin{tabular}{||l|r|r|r|r|r|r|r||}
\hline DEPT & 1 & 2 & 3 & 4 & 5 & 6 & $\mathrm{X}$ \\
\hline 3 & 0 & 496 & - & 852 & 0 & 0 & 0 \\
\hline$X$ & 532 & 0 & 0 & 0 & 716 & 616 & - \\
\hline
\end{tabular}

The resulting layout from this algorithm is 3-4-5-1-X-6-2. After rescheduling, the total material handling cost is $\$ 13,286.54$. The layout is shown in Figure 5-4. 


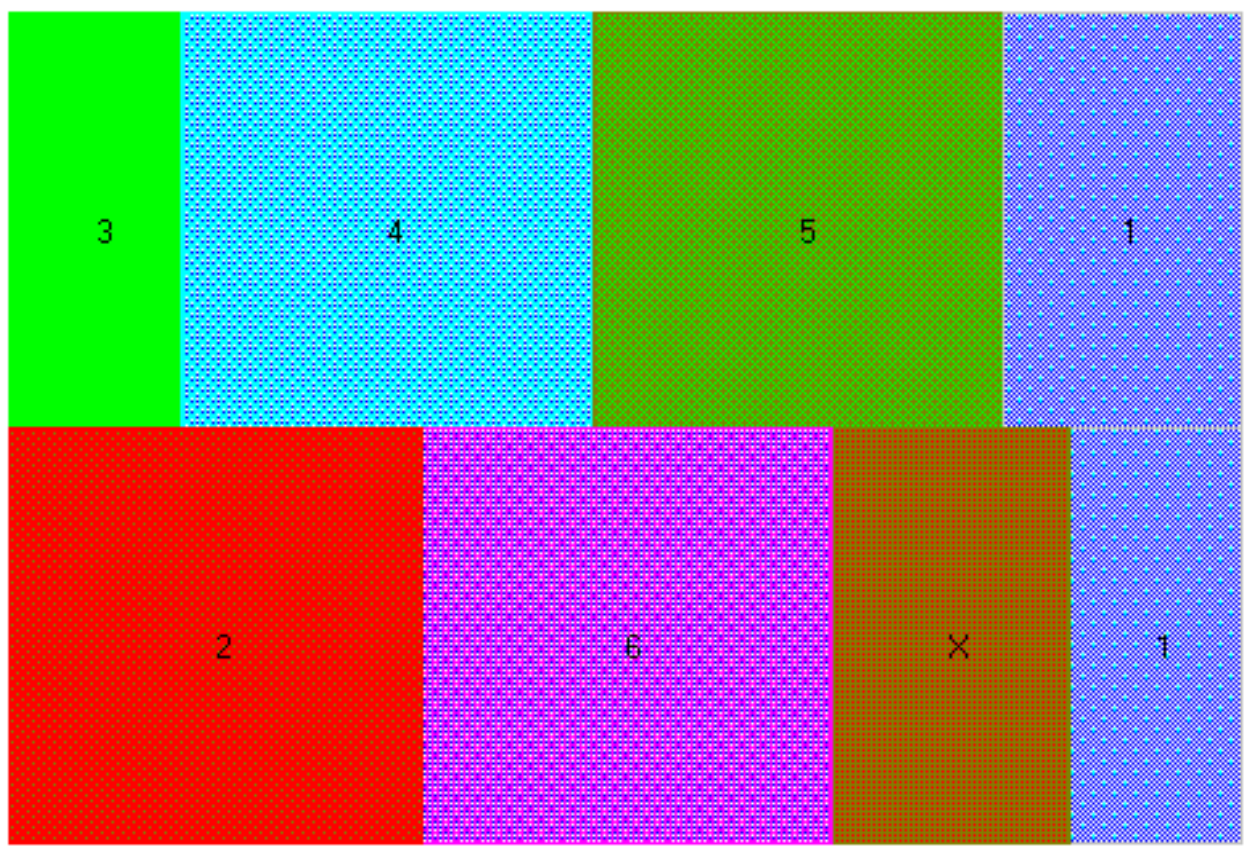

Figure 5-4 Layout After Department 3 Is Split

Compared to CRAFT, the cost saving is $14,796.40-13,286.54=\$ 1,509.86$, more than a $10 \%$ cost reduction.

Above the expected material flow is used for calculating total material handling cost. Now we consider each possible material flow and compare the results. Table 5-7 shows the total material handling cost from the CRAFT layout and the Flexible Layout Design Algorithm layout.

Table 5-7 Total Material Handling Costs

\begin{tabular}{|r|r|r|r|r||}
\hline \multicolumn{1}{|r|}{ Flow } & Split layout & CRAFT layout & Cost saving & \% cost saving \\
\hline 1 & $11,328.31$ & 13,776 & $2,447.69$ & $17.8 \%$ \\
\hline 2 & $13,144.25$ & 16,599 & $3,454.75$ & $20.8 \%$ \\
\hline
\end{tabular}




\begin{tabular}{|r|r|r|r|r||}
\hline 3 & $13,078.80$ & 13,492 & 413.20 & $3.1 \%$ \\
\hline 4 & $13,637.80$ & 14,609 & 971.20 & $6.6 \%$ \\
\hline 5 & $15,243.54$ & 15,506 & 262.46 & $1.7 \%$ \\
\hline Total & $66,432.70$ & 73,982 & $7,549.30$ & $10.2 \%$ \\
\hline
\end{tabular}

The flexible layout design algorithm can be used for multiple splits. If it continues to split, department 1 is split to departments 1 and $\mathrm{Y}$. The material flows are rescheduled as shown in Table 5-8. The resulting layout from this algorithm is 3-4-1-X-6-5-Y-2. The total material handling cost is $\$ 12,206.44$. The layout is shown in Figure 5-5.

Table 5-8 Material Flows of Departments 1 and Y

\begin{tabular}{||l|r|r|r|r|r|r|r|r||}
\hline DEPT & 1 & 2 & 3 & 4 & 5 & 6 & $\mathrm{X}$ & $\mathrm{Y}$ \\
\hline 1 & - & 0 & 0 & 667 & 0 & 0 & 532 & 0 \\
\hline $\mathrm{Y}$ & 0 & 474 & 0 & 0 & 656 & 430 & 0 & - \\
\hline
\end{tabular}

Compared to CRAFT, the cost saving is $14,796.40-12,206.44=\$ 2,589.96$, more than a $17 \%$ cost reduction.

For each possible material flow, Table 5-9 shows the total material handling cost from the CRAFT layout and the Flexible Layout Design Algorithm layout.

The cost saving is $73,982-61,032.21=\$ 12,949.79$, more than a $17 \%$ cost reduction. 


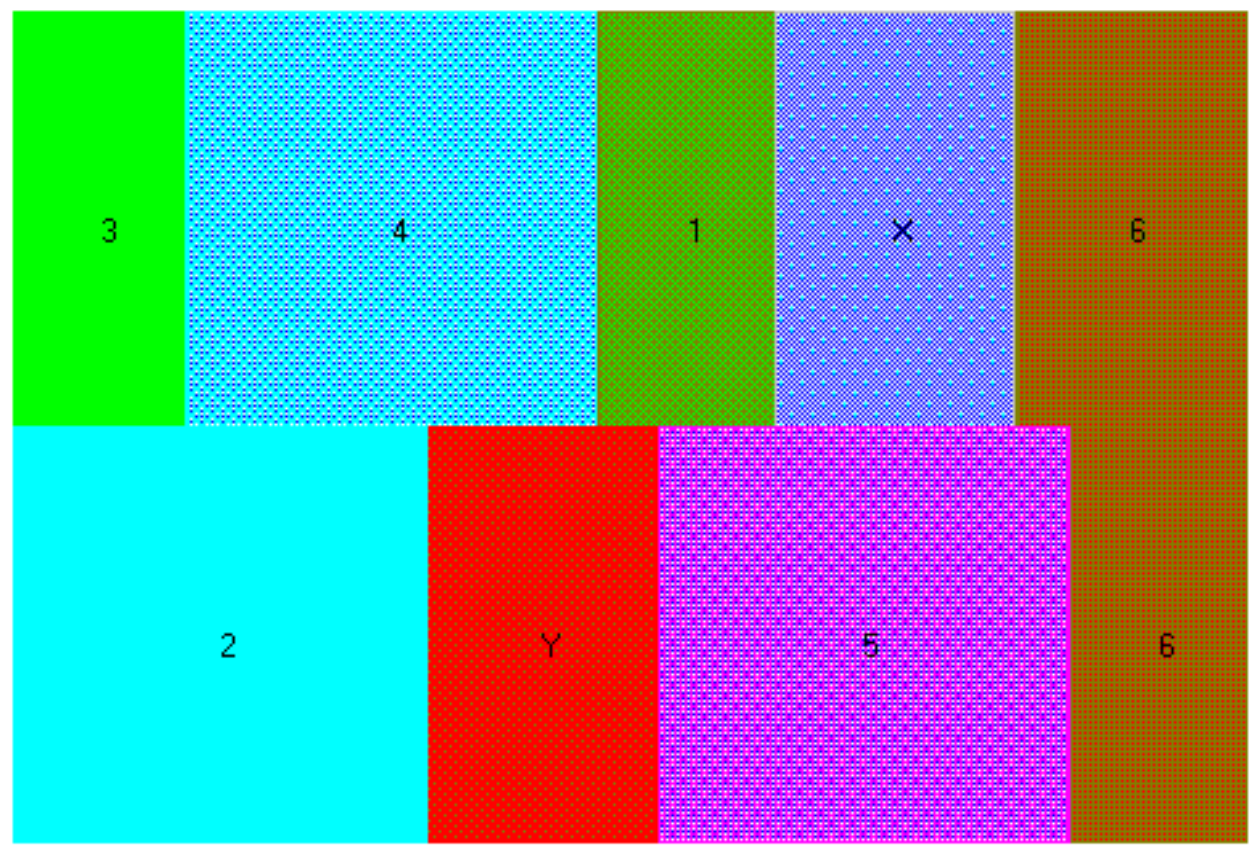

Figure 5-5 Layout After Department 1 Is Split

Table 5-9 Total Material Handling Costs

\begin{tabular}{|r|r|r||}
\hline \multicolumn{1}{|r|}{ Flow } & \multicolumn{1}{|c|}{ Split layout } & CRAFT layout \\
\hline 1 & $10,867.20$ & 13,776 \\
\hline 2 & $12,293.65$ & 13,499 \\
\hline 3 & $13,546.91$ & 14,609 \\
\hline 4 & $13,555.82$ & 15,506 \\
\hline 5 & $10,768.63$ & 73,982 \\
\hline Total & $61,032.21$ & \\
\hline
\end{tabular}


If the procedure continues, next department 6 is split to departments 6 and Z. The material flows are rescheduled as shown in Table 5-10. The resulting layout from this algorithm is 3-4-2-1-X-Z-Y-5-6. The total material handling cost is $\$ 10,731.04$. The layout is shown in Figure 5-6.

Table 5-10 Material Flows of Departments 6 and $Z$

\begin{tabular}{||l|r|r|r|r|r|r|r|r|r||}
\hline DEPT & 1 & 2 & 3 & 4 & 5 & 6 & $\mathrm{X}$ & $\mathrm{Y}$ & $\mathrm{Z}$ \\
\hline 6 & 0 & 0 & 0 & 630 & 664 & - & 0 & 0 & 0 \\
\hline $\mathrm{Z}$ & 0 & 477 & 0 & 0 & 0 & 0 & 616 & 430 & - \\
\hline
\end{tabular}

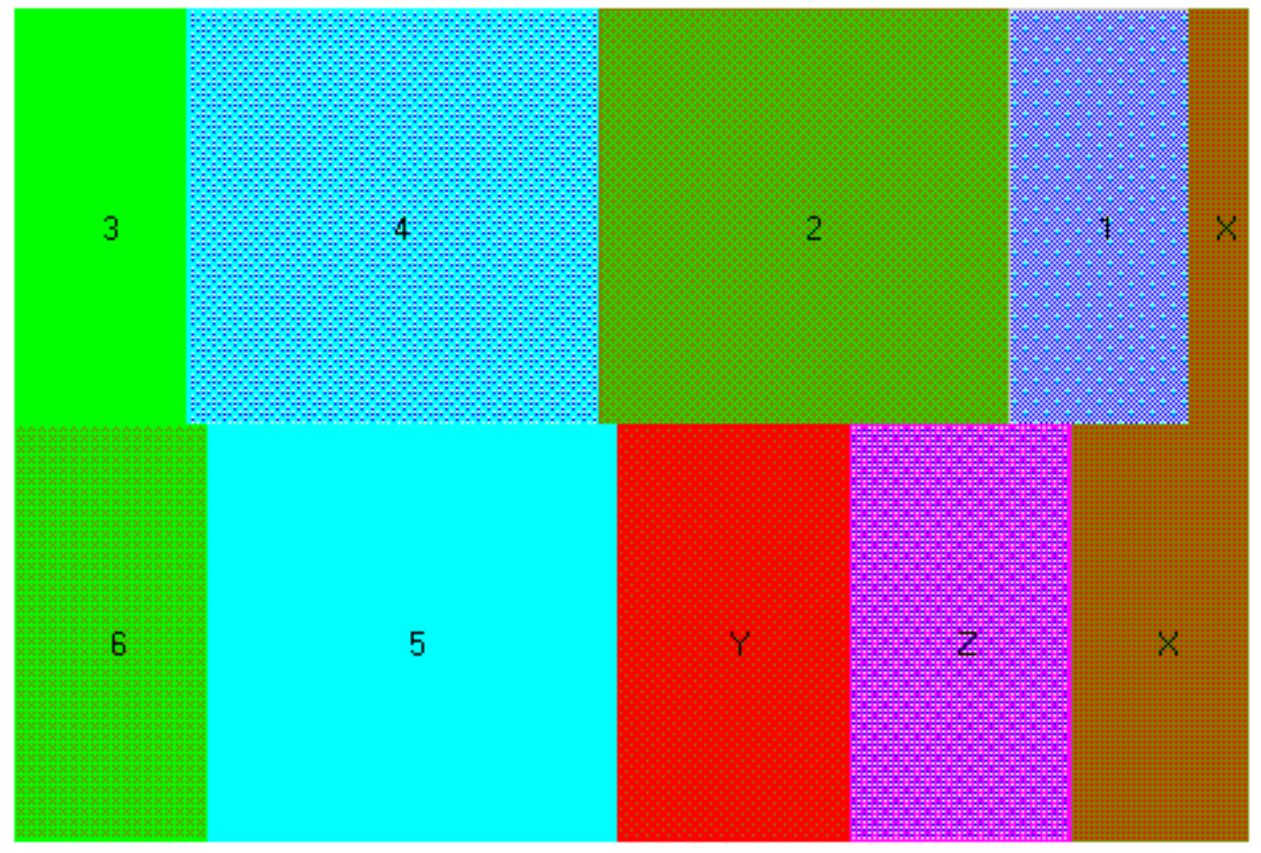

Figure 5-6 Layout After Department 6 is Split

Compared to CRAFT, the cost saving is $14,796.40-10,731.04=\$ 4,065.36$, more than a $27 \%$ cost reduction. 
For each possible material flow, Table 5-11 shows the total material handling cost from the CRAFT layout and the Flexible Layout Design Algorithm layout.

\section{Table 5-11 Total Material Handling Costs}

\begin{tabular}{|r|r|r||}
\hline \multicolumn{1}{|r|}{ Flow } & \multicolumn{1}{|c|}{ Split layout } & CRAFT layout \\
\hline 1 & $10,047.00$ & 13,776 \\
\hline 2 & $11,828.78$ & 16,599 \\
\hline 3 & $11,042.96$ & 14,492 \\
\hline 4 & $10,697.21$ & 15,506 \\
\hline 5 & $10,039.24$ & 73,982 \\
\hline Total & $53,655.19$ & \\
\hline
\end{tabular}

The cost saving is $73,982-53,655.19=\$ 20,326.81$, more than a $27 \%$ cost reduction.

\subsubsection{Comparison to CORELAP method}

CORELAP uses a relationship chart instead of a from-to chart. First the from-to chart will be converted to a relationship chart. Let's define relation $\mathrm{A}=$ over $800, \mathrm{E}=701-$ $800, \mathrm{I}=601-700, \mathrm{O}=501-600, \mathrm{U}=$ below 500. The relationship chart is shown in Figure 5-7. 


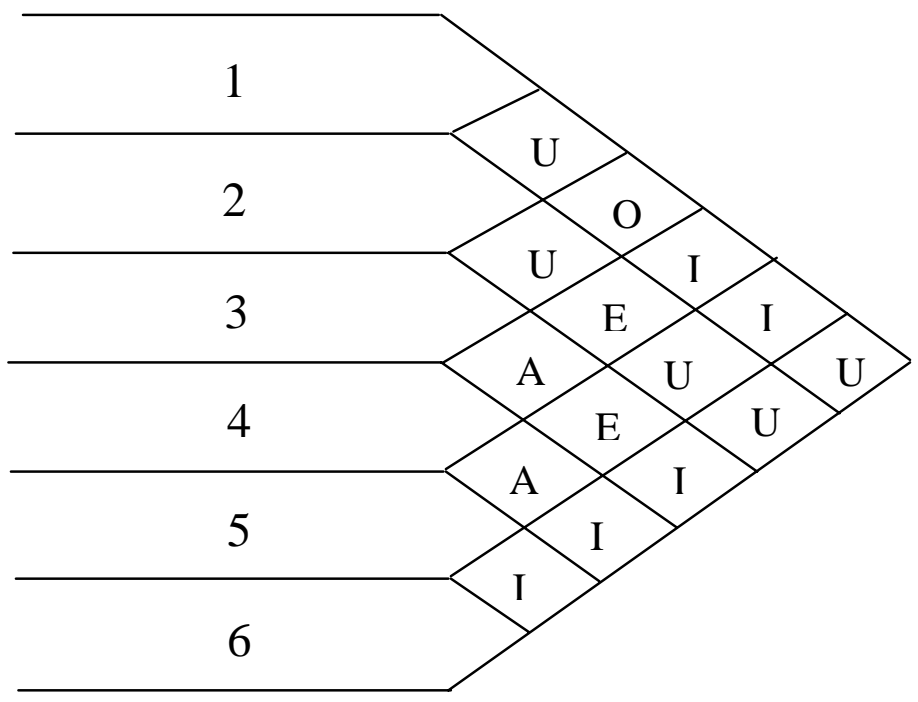

Figure 5-7 Relationship Chart

When $\mathrm{A}=6, \mathrm{E}=5, \mathrm{I}=4, \mathrm{O}=3$ and $\mathrm{U}=2$, the Total Closeness Rating(TCR) for each department is calculated as below:

\begin{tabular}{||c|c|c|c|c|c|c||}
\hline \hline DEPT. & 1 & 2 & 3 & 4 & 5 & 6 \\
\hline TCR & 15 & 13 & 20 & 25 & 21 & 16 \\
\hline
\end{tabular}

According to TCR, the first department to enter the layout is department 4. Both departments 3 and 5 have the relationship 'A' with department 4, but department 5 has a higher TCR, so it enters next. Department 3 is the third one to enter. Department 2 enters next because it has the relationship 'E' with department 4. Both departments 1 and 6 have relationship 'I' with department 4. Department 6 has higher TCR, so it enters before department 1 . So the order to enter is departments $4-5-3-2-6-1$. 
Department 4 is placed at the center of layout. Department 5 is next to it. After Placing Rating(PR) is calculated, each department has its location. The final layout is shown in Figure 5-8.

\begin{tabular}{|l|l|l|}
\hline 3 & 4 & 2 \\
\hline 6 & 5 & 1 \\
\hline
\end{tabular}

Figure 5-8 CORELAP Layout

This layout is the same as the one created by CRAFT. The total material handling cost is $\$ 73,982$. If the flexible layout design algorithm is used for just one department splitting, the cost saving is $73,982-66,432.70=\$ 7,549.30$, more than a $10 \%$ cost reduction compared to CORELAP method.

\subsubsection{Comparison to ALDEP method}

ALDEP uses the same information as CORELAP does, but it selects the first department randomly and uses the sweep method to locate departments. For this case, each department will be tried for first department to enter.

When department 1 enters first, department 4 has relationship 'I' with it, so department 4 enter next. Department 3 has relationship 'A' with department 4 . It enters third. Next is department 2 because it has relationship 'E' with department 3. Department 
5 is selected randomly because both departments 5 and 6 don't have an important relationship with department 2. Department 6 is the last one to enter. The final order is 1 $-4-3-2-5-6$. The order expression is the same as the one used by ALDEP. The layout is shown in Figure 5-9.

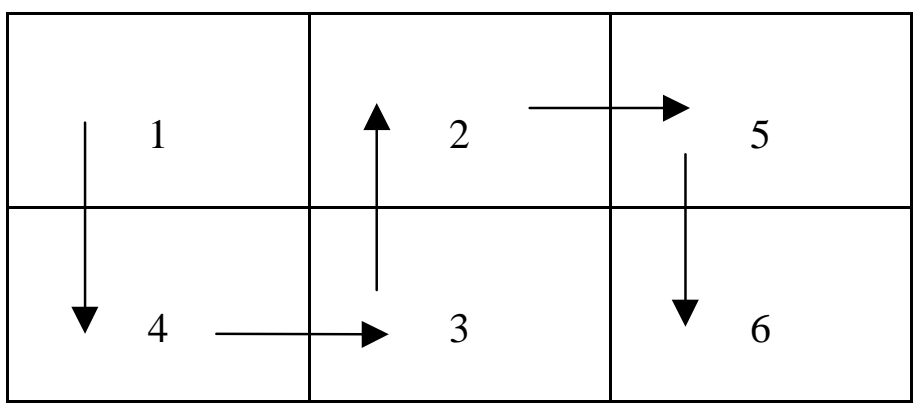

Figure 5-9 One ALDEP Layout

Similarly, when other departments are selected to enter first, the layouts and material handling costs are list in Table 5-12.

Table 5-12 ALDEP Layouts and Material Handling Costs

\begin{tabular}{||c|c|c|c|c|c|c||}
\hline Flow & & & & & & \\
\hline Layout & & & 3 & 4 & 5 & TOTAL \\
\hline $1-4-3-2-5-6$ & 14,357 & 16,471 & 17,516 & 15,417 & 14,223 & 77,984 \\
\hline $2-4-3-5-1-6$ & 13,572 & 16,507 & 15,164 & 14,793 & 16,051 & 76,087 \\
\hline $3-4-5-1-2-6$ & 15,662 & 18,365 & 14,806 & 14,907 & 14,100 & 77,840 \\
\hline $4-3-5-1-2-6$ & 15,231 & 17,638 & 15,162 & 14,414 & 13,867 & 76,312 \\
\hline
\end{tabular}




\begin{tabular}{||c|c|c|c|c|c|c||}
\hline $5-4-3-6-1-2$ & 14,117 & 16,523 & 15,644 & 15,265 & 15,859 & 77,408 \\
\hline $6-3-4-5-1-2$ & 13,776 & 16,599 & 13,492 & 14,609 & 15,506 & $\underline{73,982}$ \\
\hline
\end{tabular}

Therefore, the best layout from ALDEP is $6-3-4-5-1-2$. The layout is shown in Figure 5-10.

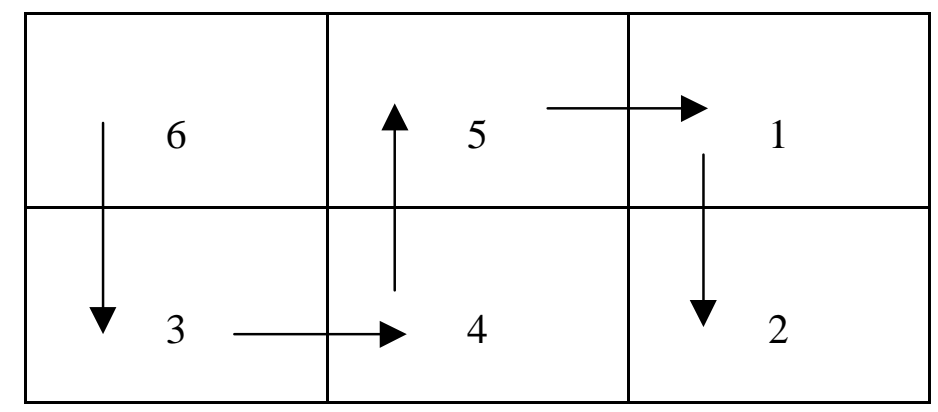

Figure 5-10 The Best ALDEP Layout

The total material handling cost is $\$ 73,982$. If the flexible layout design algorithm is used for just one department splitting, the cost saving is $73,982-66,432.70=\$ 7,549.30$, more than a $10 \%$ cost reduction compared to ALDEP method.

\subsubsection{Comparison to some publishing}

In [Rosenblatt, 1986], the optimal solution is layout $(2,4,6,1,3,5)$ for stages 1 and 2 ; $(2,4,6,1,5,3)$ for stage $3 ;(2,6,4,1,5,3)$ for stage 4 ; and $(2,1,4,6,5,3)$ for stage 5 . The total material handling cost is $\$ 71,187$. The result of the author's heuristic algorithm is layout $(6,3,1,5,4,2)$ for stages 1 and 2 ; and $(6,5,1,3,4,2)$ for stages 3,4 and 5 . The total material handling cost is $\$ 72,228$. By using the flexible split layout design algorithm, the cost savings are $71,187-66,432.70=\$ 4,754.30$ or $6.7 \%$, and $72,228-66,432.70=\$ 5,795.30$ 
or $8.0 \%$, respectively. Furthermore, there is only one single layout. It is not necessary to relocate the departments for each stage.

In [Rosenblatt, 1992], it assumed the probabilities for each future flow are 0.3, 0.1, $0.05,0.15$ and 0.4 , respectively. The layout is $(2,1,5,6,3,4)$. The total material handling cost is $\$ 14,573$. It also used the method in [Shore, 1980] for comparison. That result is $(1,4,2,6,3,5)$. The total material handling cost is $\$ 14,625$. Under the assumption of these probabilities, the cost by using the flexible split layout design algorithm to split only one department is $0.3 * 11,328.31+0.1 * 13,144.25+0.05 * 13,078.80+0.15 * 13,637.80+$ $0.4 * 15,243.54=\$ 13,509.94$. The cost savings are $14,573-13,509.94=\$ 1,063.06$ or $7.3 \%$, and $14,625-13,509.94=\$ 1,115.06$ or $7.6 \%$, respectively.

Furthermore, we can try some other probabilities for each future flow for more comparisons. Table 5-13 lists the material handling cost for different flows. Table 5-14 lists the expected material handling cost for different probabilities. From the results we can see that the cost reduction is very significant compared to these current algorithms for all of these different distributions. So the layout created by the flexible layout design method is flexible.

Table 5-13 Material Handling Costs for Different Flows

\begin{tabular}{||l|l|l|l|l|l||}
\hline & Flow 1 & Flow 2 & Flow 3 & Flow 4 & Flow 5 \\
\hline Split Layout & $11,328.31$ & $13,144.25$ & $13,078.80$ & $13,637.80$ & $15,243.54$ \\
\hline Rosenblatt & 13,694 & 16,269 & 17,046 & 15,711 & 14,072 \\
\hline Shore & 12,964 & 14,853 & 14,962 & 16,165 & 15,193 \\
\hline
\end{tabular}


Table 5-14 Expected Material Handling Costs for Different Probabilities

\begin{tabular}{|l|l|l|l|l|r|r|r|r|r||}
\hline \hline Flow 1 & Flow 2 & Flow 3 & Flow 4 & Flow 5 & Split Layout & Rosenblatt & $\begin{array}{l}\text { Cost } \\
\text { savings } \\
(\%)\end{array}$ & Shore & \multicolumn{1}{l|}{$\begin{array}{l}\text { Cost } \\
\text { savings } \\
(\%)\end{array}$} \\
\hline 0.3 & 0.1 & 0.05 & 0.15 & 0.4 & $13,509.94$ & $14,573.00$ & 7.30 & $14,625.00$ & 7.60 \\
\hline 0.2 & 0.2 & 0.2 & 0.2 & 0.2 & $11,248.54$ & $15,358.40$ & 26.76 & $14,827.40$ & 24.14 \\
\hline 0.1 & 0.3 & 0.3 & 0.2 & 0.1 & $13,251.66$ & $15,913.30$ & 16.73 & $14,993.20$ & 11.62 \\
\hline 0.05 & 0.15 & 0.4 & 0.3 & 0.1 & $13,385.27$ & $16,063.95$ & 16.68 & $15,229.75$ & 12.11 \\
\hline 0.15 & 0.15 & 0.2 & 0.25 & 0.25 & $13,506.98$ & $15,349.40$ & 12.00 & $15,004.45$ & 9.98 \\
\hline 0.3 & 0.15 & 0.1 & 0.25 & 0.2 & $13,136.17$ & $14,995.30$ & 12.40 & $14,693.20$ & 10.60 \\
\hline
\end{tabular}

\subsection{Forecasting Validation}

One case study is to modify the case presented in 5.3. The data were changed slightly different from their original values. All five flow sets were checked. The results are reasonable.

Another case study is taken from J. Heizer and B. Render, Production and Operations Management, $4^{\text {nd }}$ edition, 1996, pp171-173, Example 6. The results are shown in Table 5-15. These results are better than the solution in the literature. 
Table 5-15 Forecasting Results

\begin{tabular}{||l|l|l|l||}
\hline MONTH & $\begin{array}{l}\text { ACTUAL } \\
\text { DEMAND }\end{array}$ & $\begin{array}{l}\text { FORECAST } \\
\text { BY HEIZER }\end{array}$ & $\begin{array}{l}\text { FORECAST BY } \\
\text { FORECASTING MODULE }\end{array}$ \\
\hline 1 & 12 & 11.00 & 12.77 \\
\hline 2 & 17 & 11.28 & 15.60 \\
\hline 3 & 20 & 12.87 & 18.43 \\
\hline 4 & 19 & 14.81 & 21.26 \\
\hline 5 & 24 & 15.87 & 24.09 \\
\hline 6 & 26 & 18.03 & 26.92 \\
\hline 7 & 31 & 20.10 & 29.72 \\
\hline 8 & 32 & 22.98 & 32.58 \\
\hline 9 & 36 & 25.27 & 35.41 \\
\hline 10 & - & 28.03 & 38.24 \\
\hline
\end{tabular}

\subsection{Evaluation Validation}

Because there is no similar work done about this kind of evaluation, the case study is to modify the case presented in 5.3. The sequences of operations are shown in the Table 5-16.

Table 5-16 Sequences of Operations

\begin{tabular}{|c|c|c|c|c|}
\hline Part no & Dept sequence & Material & Quantity mean & Quantity variance \\
\hline 1 & 123456 & 3 & 474 & 10 \\
\hline 2 & 154263 & 5 & 339 & 9 \\
\hline 3 & 325146 & 7 & 22 & 1 \\
\hline 4 & 625314 & 2 & 138 & 3 \\
\hline 5 & 56413 & 8 & 190 & 4 \\
\hline 6 & 31425 & 9 & 204 & 4 \\
\hline
\end{tabular}




\begin{tabular}{|c|c|c|c|c|}
\hline 7 & 416352 & 4 & 113 & 3 \\
\hline 8 & 253461 & 6 & 42 & 1 \\
\hline 9 & 6351 & 1 & 164 & 3 \\
\hline 10 & 51643 & 5 & 131 & 3 \\
\hline 11 & 16435 & 8 & 144 & 3 \\
\hline 12 & 435 & 7 & 61 & 2 \\
\hline 13 & 35 & 3 & 54 & 2 \\
\hline 14 & 642 & 9 & 101 & 3 \\
\hline 15 & 24 & 2 & 135 & 3 \\
\hline
\end{tabular}

If the above sequences of operations are converted to From-To material flow, it is the same as the From-To chart of the case in 5.3, so we can compare their results. For the layout created by the Flexible Layout Design Algorithm with splitting one department, first the Resequence module is used to re-sequence the flow to shorten the distance. Then the Evaluation module is used to calculate the mean and variance of the total material handling cost. The results and comparisons are shown in Table 5-17.

Table 5-17 Evaluation Results

\begin{tabular}{|c|c|c|c|c|c|}
\hline Methods & MHCost Mean & $\begin{array}{l}\text { MHCost } \\
\text { Variance }\end{array}$ & $\begin{array}{l}\text { Standard } \\
\text { Deviation }\end{array}$ & $\begin{array}{c}\text { 0.9544 Prob. } \\
\text { Interval } \\
\text { (Normal Dist.) } \\
\mu \pm 2 \sigma\end{array}$ & $\begin{array}{c}\text { 0.888 Prob. } \\
\text { Interval } \\
\text { (General Dist.) } \\
\mu \pm 3 \sigma\end{array}$ \\
\hline $\begin{array}{l}\text { Flexible } \\
\text { Layout }\end{array}$ & $13,371.46$ & $1,988.28$ & 44.59 & $\begin{array}{l}13,282.28 \\
13,460.64\end{array}$ & $\begin{array}{l}13,237.69 \\
13,505.23\end{array}$ \\
\hline CRAFT & $14,797.00$ & $2,509.00$ & 50.09 & $\begin{array}{l}14,696.82 \\
14,897.18\end{array}$ & $\begin{array}{l}14,646.73 \\
14,947.27\end{array}$ \\
\hline $\begin{array}{c}\text { Rosenblatt } \\
\text { (1992) }\end{array}$ & $15,665.00$ & $2,621.00$ & 51.20 & $\begin{array}{l}15,562.60 \\
15,767.40\end{array}$ & $\begin{array}{l}15,511.40 \\
15,818.60\end{array}$ \\
\hline $\begin{array}{l}\text { Shore } \\
\text { (1980) }\end{array}$ & $15,196.00$ & $2,727.00$ & 52.22 & $\begin{array}{r}15,091.56 \\
15,300.44\end{array}$ & $\begin{array}{r}15,039.34 \\
15,352.66\end{array}$ \\
\hline
\end{tabular}


From Table 5-17 we can find that, first, the total material handling cost's mean by the Flexible Layout Design Algorithm is the least. The layout is efficient. Second, the total material handling cost's variance is also the least. That means the total material handling cost falls into a small interval of value. The layout by the Flexible Layout Design Algorithm is less sensitive to a change of material flows. It is flexible.

\subsection{Conclusion}

In this research, the major emphasis is on the validation of the Flexible Layout Design Algorithm. It must be noted that in order to validate the algorithm the whole computer program and each module have all been validated. The validation procedure demonstrates the robustness of the computer program. The validation results demonstrate that the algorithms built into the program function and yield better results in all of the cases when comparisons are made with the already available data. 


\section{CONTRIBUTIONS OF THE RESEARCH}

The Layout Design System presented in this dissertation has the following major contributions.

\subsection{Algorithm}

The Flexible Layout Design Algorithm is a new method in layout design. The algorithm designs a hybrid layout that takes advantage of process layout and cellular layout. No similar work has been done before. The layout created has two major advantages. By splitting one department into two or more portions like workstations, this heuristic method first shortens the distance among the departments. Hence it reduces the total material handling cost. So it is efficient. Secondly, it provides the opportunity to reschedule the future material flow in spite of their uncertainty at the design stage. It is the material flow that accommodates the layout, not the layout that accommodates the material flow. This ensures that the layouts created have lower total material handling cost and are able to handle disturbances caused by future events. The way that the algorithm integrates the scheduling into its layout design procedure is also a contribution because no other methods functioned this way. The layout created by the Flexible Layout Design Algorithm has better results than those in current literature as well as by some famous algorithms. This algorithm will help plant layout designers to analyze and design efficient and flexible layouts with lower total material handling cost. 


\subsection{Evaluation}

In both traditional and current study, distribution of the material flow is not considered in layout design. The problem is that usually the material From-To flows are used in layout design. That means the flow is between two departments. The joint distribution for these flows is very complicated because they are not independent. So for certain material flow situation, previous works just used the total material handling cost for the evaluation criterion. For uncertain material flow situations, previous work usually used the simulation for evaluation, or just assumed specific probabilities for the material flow.

In this research, sequences of operations of parts are used instead of material FromTo flow between two departments. This is reasonable because they match the processes of parts in a plant. The sequences of operations can be converted to From-To flows. They are consistent. All layout design methods that use the From-To flow chart still work with this input option. The CRAFT algorithm also has the sequences of operations option. Material From-To flows should not be independent of each other because usually materials go through more than two departments. It is not reasonable to assume that every part only has two operations in two departments, but it is reasonable to assume that the sequences of operations are independent of each other because they belong to different parts.

With sequences of operations we can evaluate layouts by using probability distribution. This is a new method in layout evaluation. The total material handling cost 
is a function of all sequences of operations. With or without assuming normal distribution of sequences of operations, the mean and variance of the total material handling cost can be calculated. These two parameters and the probability interval are used to evaluate the layouts. This problem definition and evaluation method are new for layout design. These results under stochastic conditions are very helpful when uncertain material flow is considered.

\subsection{Integration}

The whole layout design system integrates all the related functions of forecasting, pair exchange layout design algorithm, flexible layout design algorithm, layout evaluation, layout drawing, scheduling and data converting. A database is used for the interaction of these functions. There are not any other layout design systems that have such complete functions. Some of the functions such as forecasting, layout evaluation, layout drawing and data converting can be used for layouts created by other methods. The forecasting and data converting functions can even be used for purposes other than layout design.

\subsection{Conclusion}

Above is a summary of the major contributions in this research. All these contributions focus on the flexible layout design problems. This new work can create better results with lower total material handling cost, proved by some case studies. These contributions should be helpful in the research of plant layout design. 


\section{CONCLUSIONS AND FUTURE WORK}

The developed algorithms and computer system presented in this research illustrate the significance of the design for flexible layout. The study performed in this research, along with the developed computer system, is extremely useful for researchers and designers to appreciate the effect of layout design decision, especially flexible layout design. When a layout is designed, it is not only from the perspective of material flows and department information, but also from the perspective of forecasting, scheduling and evaluation. As far as material flow is concerned, not only deterministic situations are considered, but also indeterministic situations as well. The layout results in a low total material handling cost and flexibility regarding disturbance in the future, and helps the industry to maintain a competitive edge.

The research study presented here has investigated the effects of the algorithms on the layout design and other functions related to the layout design. Maximum effort has been taken to develop a new flexible layout design system that includes and integrates forecasting, pair exchange layout design algorithm, flexible layout design algorithm, layout evaluation, layout drawing, scheduling and data converting. The system is implemented by a computer program that includes a database and more than ten modules. Some of the modules can be used not only for flexible layout design, but also for other layout design analysis, even other analysis rather than layout design. 
While this research has made every attempt to encompass all major areas, there is always room to incorporate more details or factors, as part of future work. Some of them are outlined below:

1. In the Evaluation module, there is an assumption that the schedule of one sequence of operations doesn't affect other schedules. Capacity of departments is not considered in the Evaluation module. But in reality the departments have limited capacity. How to involve the capacity in the evaluation can be studied in the future research.

2. In this research, the building is assumed to be rectangular. While this is a common feature in certain industries, there are situations when a building is required to have different dimensions. Future research could incorporate such a feature.

3. The current research assumes all material movement is between department centers. This is a very common assumption in layout approach. But in reality, receiving and shipping stations may be at the boundaries of departments. More work can be done in the future on how to handle this kind of situation.

4. The travel distances are rectilinear or Euclidean, an abstract route of material handling equipment. The material handling system is represented by material moving cost. Future research could integrate practical material handling system design into layout design. This would make the approach closer to industry application. 
5. Basically, this research is a methodological approach. In a practical plant, many qualitative factors need to be considered as well as total material handling cost. Some of these are aisle arrangement, office requirements, and personnel requirements (like lockers, restrooms, food services, health services, etc.). In future studies a computer-aided drawing tool should be developed to assist the designer to modify the layout created by the algorithm.

6. Currently only one forecasting model is used. Other forecasting models can be included in this research in the future to let the users have more options in forecasting.

7. This study is for single-floor layout. Multiple-floor layout situations could be considered in future research. In that case, material movement among floors would be an important factor in layout design.

8. The goal of current research is to design a hybrid layout that is efficient and flexible in case of future disturbance. The layout incorporates the features of process layout and cellular layout. If the plant is limited to locating all equipment within a department together, this approach is not suitable. In this case, other approaches should be developed. One possible approach is to arrange several areas for expansion of departments. A group of departments shares one area. The departments are grouped based on a rule that these departments will not expand or shrink together. Future study can be on how to design these groups and areas and how to utilize these areas before they are used by expansion of departments. 


\section{REFERENCES}

1. Afentakis, P., Millen, R.A., et al, Dynamic layout strategies for flexible manufacturing systems, International Journal of Production Research, 28, 2, 1990, pp311-323.

2. Allred, J.K., Taking a New Direction in Factory Logistics and Material Handling, IIE Solutions, 28, April 1996, pp21-25.

3. Ang, C.L., Willey, P.C.T., A comparative study of the performance of pure and hybrid group technology manufacturing systems using computer simulation techniques, International Journal of Production Research, 22, 1984, pp193-233.

4. Askin, R.G, Standridge, C.R., Modeling and analysis of Manufacturing Systems, Wiley, NY, 1993.

5. Automotive Stamper Designs Plant Layout based on JIT, IIE Solutions, 29, Jan. 1997, pp48-49.

6. Balakrishnan, J., Notes: The Dynamics of Plant layout, Management Science, 39, 5, 1993. pp654-655.

7. Bauer, A., Bowden, R., et al, Shop Floor Control Systems, Chapman \& Hall, London, UK, 1991. 
8. Black, J.T., Jiang, B.C., et al, Design, Analysis, and Control of Manufacturing Cells, American Society of Mechanical Engineers, NY, 1991.

9. Rembold, U., Blume, C., et al, Computer Integrated Manufacturing Technology and Systems, M.Dekker, NY, 1985.

10. Bozer, Y.A., Meller, R.D., A reexamination of the distance-based facility layout problem, IIE Transaction, 29, 7, 1997. pp549-560.

11. Bozer, Y.A., Meller, R.D., et al, An Improvement-type Layout Algorithm for Single and Multiple-floor Facilities, Management Science, 40, 7, 1994, pp918-932.

12. Buffa, E.S., Armour, G.C., et al, Allocating Facilities with CRAFT, Harvard Business Review, 42, 2, 1964. pp136-158.

13. Bussey, L.E., Eschenbach, T.G., The Economic Analysis of Industrial Projects, 2nd edition, Prentice Hall, NJ, 1992.

14. Dahel, N.E., Smith, S.B., Designing flexibility into cellular manufacturing systems, International Journal of Production Research, 31, 4, 1993, pp933-945.

15. Design plans \& ideas, Modern Materials Handling, v51, supplement to Mar., 1996, pps3-s24.

16. Design plans \& ideas, Modern Materials Handling, v51, supplement to Jan., 1996.

17. Dieter, G.E., Engineering Design, 2nd Edition, McGraw-Hill, NJ, 1991. 
18. Drezner, Z., DISCON: a new Method for the layout problem, Operations Research, 28, 6, 1980, pp1375-1384.

19. Facilities Planning Software Buyer's Guide, IIE Solutions, Jan. 1998, pp44-48.

20. Facilities Planning Software Buyer's Guide, IIE Solutions, Jan. 1997, pp40-43.

21. Facilities Planning Software Buyer's Guide, IIE Solutions, July 1996, pp40-43.

22. Facilities Planning Software Buyer's Guide, IIE Solutions, Aug. 1995, pp48-51.

23. Fink, P.K., An Intelligent Facility Planning Adviser, Artificial Intelligence for Engineering, Design, and Manufacturing, ISA(Instrument Society of America) Transactions, 31, 2, 1992, pp77-95.

24. Flynn, B.B., Jacobs, F.R., An experimental comparison of cellular(group technology) layout with process layout, Decision Sciences, 18, 4, 1987, pp562-581.

25. Foulds, L.R., Gibbons, P.B., et al, Facilities layout adjacency determination: an experimental comparison of three graph theoretic heuristics, Operations Research, 33, Sep.-Oct. 1985, pp1091-1106.

26. Francis, R.L., White, J.A., Facility Layout and Location, Prentice-Hall, NJ, 1974.

27. Fu, M.C., Kaku, B.K., Minimizing work-in-process and material handling in the facilities layout problem, IIE Transaction, 29, 1, 1997. pp29-36. 
28. Gerwin, D., Manufacturing Flexibility: A Strategic Perspective, Management Science, 39, 4, 1993. pp395-410.

29. Gopalakrishnan, B., Product Design and Process Planning in Concurrent Engineering, ISPE, 1996.

30. Graybeal, W.J., Simulation: Principles and Methods, Winthrop Publishers, Cambridge, 1980.

31. Gupta, R.M., Flexibility in Layouts: A simulation approach, Material Flow, 3, 4, June 1986, pp243-250.

32. Gupta, R.M., Tompkins, J.A., An examination of the dynamic behavior of part families in group technology, International Journal of Production Research, 20, 1982, pp73.

33. Hales, H.L., Computer-Aided Facilities Planning, M. Dekker, NY, 1984.

34. Hassan, M.M.D., Machine layout problem in modern manufacturing facilities, International Journal of Production Research, 32, 11, 1994. pp2559-2584.

35. Hassan, M.M.D., Hogg, G.L., et al, SHAPE: a Construction Algorithm for Area Placement Evaluation, International Journal of Production Research, 24, 5, 1986, pp1283-1295.

36. Heizer, J., Render, B, Production \& Operations Management, 4th, Prentice Hall, NJ, 1996. 
37. Heragu, S.S., Kusiak, A., Efficient Models for the Facility Layout Problem, European Journal of Operational Research, 53, 1991, pp1-13.

38. Hills, W., Barlow, M., and et al, Layout Design of Large Made-to-order Products using a Knowledge-based System, Journal of Engineering Manufacture, 207, 4, 1993, pp257-263.

39. Hoover, S.V., Perry, R.F., Simulation, Addison-Wesley, Mass., 1989.

40. Hunt, V.D., Reengineering, Omneo, VT, 1993.

41. Hurrion, R.D., Simulation, Springer-Verlag, NY, 1986.

42. Irani, S.A., Cavalier, T.M., et al, Virtual Manufacturing Cells: exploiting layout design and intercell flows for the machine sharing problem, International Journal of Production Research, 31, 4, 1993, pp791-810.

43. Jajodia, S., Minis, L., et al, CLASS: Computerized LAyout Solutions using Simulated annealing, International Journal of Production Research, 30, 1, 1992, pp95-108.

44. James, R.W., Alcorn, P.A., A Guide to Facilities Planning, Prentice Hall, NJ, 1991

45. Jennings, R., Using Microsoft Access 97, Que Corporation, 1997

46. Johnson, R.V., SPACECRAFT for Multi-Floor Layout Planning, Management Science, 28, 4, April 1982. pp407-417. 
47. Kamoun, M., Yano, C.A., Facility Layout to Support Just-In-Time, Transportation Science, 30, 4, Nov. 1996, pp315-329.

48. Katzel, J., Layout Planning Tool Simplifies Development of Block Plans, Plant Engineering, 41, Feb. 1987, pp66-68.

49. Kinney, H.D., McGinnis, L.F., Design and control of manufacturing cells, Industrial Engineering, 19, 10, 1987, pp28-38.

50. Koenig, D.T., Manufacturing Engineering, 2nd edition, Taylor \& Francis, Washington, DC, 1994.

51. Konz, S., Facility Design, Wiley, NY, 1985.

52. Kouvelis, P., Kiran, A.S., Single and multiple period layout models for automated manufacturing systems, European Journal of Operational Research, 52, 3, 1991. pp300-314.

53. Kusiak, A., Intelligent Manufacturing Systems, Prentice Hall, NJ, 1990.

54. Kusiak, A., Heragu, S.S., Machine layout problem in flexible manufacturing systems, Operations Research, 36, 1988, pp258-268.

55. Lacksonen, T.A., Static and Dynamic Layout Problems with Varying Areas, Journal of the Operational Research Society, 45, Jan. 1994, pp59-69. 
56. Langevin, A., Montreuil, B., et al, Spine Layout Design, International Journal of Production Research, 32, 2, 1994, 429-442.

57. Lee, R.C., Moore, J.M., Corelap --- Computerized Relationship Layout Planning, Journal of Industrial Engineering, 18, 3, 1967, pp195-200.

58. Leon, V.J., Wu, S.D., et al, Robustness measures and robust scheduling for job shops, IIE Transaction, 26, 5, 1994. pp32-43.

59. Leung, L.C., Tanchoco, J.M.A., Multiple machine replacement within an integrated system framework, The Engineering Economist 32, 1987, pp89-114.

60. Liggett, R.S., Mitchell, W.J., Optimal Space Planning in Practice, Computer Aided Design, 13, 5, 1981, pp277-288.

61. Luggen, W.W., Flexible Manufacturing Cells and Systems, Prentice Hall, NJ, 1991.

62. Meller, R.D., Gau, K.Y., Facility layout objective functions and robust layouts, International Journal of Production Research, 34, 10, 1996. pp2727-2742.

63. Moder, J.J., Elmaghraby, S.E., Handbook of Operations Research, Van Nostrand Reinhold, NY, 1978.

64. Montgomery, D.C., Design and Analysis of Experiments, 3rd edition, 1991. pp414433. 
65. Montreuil, B., Venkatadri, U., Strategic interpolative design of dynamic manufacturing systems layouts, Management Science, 37, 6, 1991. pp682-694.

66. Montreuil, B., Venkatadri, U., et al, Generating a layout from a design skeleton, IIE Transaction, Jan. 1993.

67. Moodie, C., Uzsoy, R., et al, Manufacturing Cells, Taylor \& Francis, PA, 1995.

68. Moore, J.M., Plant Layout and Design, Macmillan, NY, 1962.

69. Muther, R., Systematic Layout Planning, 2nd edition, Cahners books, Boston, 1973.

70. Nicol, L.M, Hollier, R.H., Plant Layout in Practice, Material Flow, 1, 3, 1983, pp177-188.

71. Nof, S.Y., Moodie, C.L., Flow Control Effects on Facilities Planning, Material Flow, 1, 2, Mar. 1983.

72. Palekar, U.S., Planning and Control of Material Handling Systems, ASME, NY, 1991.

73. Peterson, L.D., How to tell if your manufacturing operations are non competitive, Industrial Engineering, 22, Jan. 1990. pp41.

74. Picone, C.J., Wilhelm, W.E., A Perturbation Scheme to Improve Hillier's Solution to the Facilities Layout Problem, Management Science, 30, Oct. 1984, pp1238-1249. 
75. Rosenblatt, M.J., The Dynamics of Plant Layout, Management Science, 32, 1, 1986. pp76-86.

76. Rosenblatt, M.J., Kropp, D.H., The single Period Stochastic Plant Layout Problem, IIE Transaction, 24, 2, 1992. pp169-176.

77. Rosenblatt, M.J., Lee, H.L., A Robustness Approach to Facilities Design, International Journal of Production Research, 25, 4, 1987. pp479-486.

78. Seehof, J.M., Evans, W.O., Automated Layout Design Program, the Journal of Industrial Engineering, 18, 12, 1967, pp690-695.

79. Shang, J.S., Robust design and optimization of material handling in an FMS, International Journal of Production Research, 33, 9, 1995. pp2437-2454.

80. Shore, R.H., Tompkins, J.A., Flexible Facilities Design, AIIE Transactions, 12, 2, 1980. pp200-205.

81. Sly, D., Computerized Facilities Design and Management, IIE Solutions 27, Aug. 1995, pp43-47.

82. Sly, D., Polashek, E., Fairtron scores big with new cabinet production layout redesign, Industrial Engineering, 25, Mar. 93, pp34-36.

83. Sly, D.P., Using CAD for Space Planning and Asset Management, IIE Solutions, 28, May 1996, pp36-45. 
84. Sly, D.P., Issues and Techniques for using CAD to Draw Factory Layouts, IIE Solutions, 28, Mar. 1996, pp14-18.

85. Sly, D.P., Grajo, E., and et al, Layout Design and Analysis Software, IIE Solutions, 28, July 1996, pp18-25.

86. Smith, J.M., Application of State-dependent Queues to Pedestrian/vehicular Network Design, Operations Research, 42, 3, May 94, pp414-427.

87. Smith, J.M., Bouanaka, B., Queueing Network Decomposition and Facilities Planning, Computers \& Operations Research, 12, 1, 1985, pp1-16.

88. Socha, J., Hall, D., Visual Basic 4.0, MIS:Press, 1995.

89. Smith, J.M., Graves, R.J., and et al, QNET: An Open Queueing Network Model for Material Handling System Analysis, Material Flow, 3, 4, June 1986, pp225-242.

90. Special, Modern Materials Handling, 51, 5, mid-April, 1996, pp17-102.

91. Sweeney, D.S., Tatham, R.L., An Improved Long-Run Model for Multiple Warehouse Location, Management Science, 22, 7, 1976. pp748-758.

92. Thuesen, G.J., Fabrycky, W.J., Engineering Economy, 8th edition, Prentice Hall, NJ, 1993.

93. Tam, K.Y., $\quad$ A Simulated Annealing Algorithm for Allocating Space to Manufacturing Cells, International Journal of Production Research, 30, 1, 1992, pp63-87. 
94. Tamashunas, V.M., Labban, et al, Interactive graphics offer an analysis of plant layout and material handling systems, Industrial Engineering, 22, Jan. 90, pp38-43.

95. Tompkins, J.A., Modularity and flexibility: dealing with future shock in facilities design, Industrial Engineering, Sep. 1980, pp78-81.

96. Tompkins, J.A., Schaffer, B., High-Performance Material Handling Trends, IIE Solutions, 28, April 1996, pp16-19.

97. Tompkins, J.A., Spain, J.D., Utilization of spine concept maximizes modularity in facilities planning, Industrial Engineering, 15, 3, 1983, pp34-42.

98. Tompkins, J.A., White, J.A., et al, Facilities Planning, 2nd edition, John Wiley \& Sons, NY, 1996.

99. Tompkins, J.A., White, J.A., Facilities Planning, Wiley, NY, 1984.

100. Usher, J.S., Ciesielski, C.A., et al, Redesigning an existing layout presents a major challenge -- and produces dramatic results, Industrial Engineering, June, 1990, pp45-59.

101. Vakharia, A.J., Kaku, B.K., Redesigning a Cellular Manufacturing System to Handle Long-Term Demand Changes: A Methodology and Investigation, Decision Sciences, 24, 5, 1993. pp909-930. 
102. Walpole, R.E., Myers, R.H., Probability and Statistics for Engineers and Scientists, 5th edition, Macmillan, NY, 1993.

103. Webster, D.B., Murty, S.V., Computer-based FMS facilities design tool, International Journal of Production Research, 30, 10, 1992. pp2439-2455.

104. Webster, D.B., Tyberghein, M.B., Measuring flexibility of job-shop layouts, International Journal of Production Research, 18, 1, 1980. pp21-29. 


\section{APPENDIX}


APPENDIX - A. USER INTERFACE

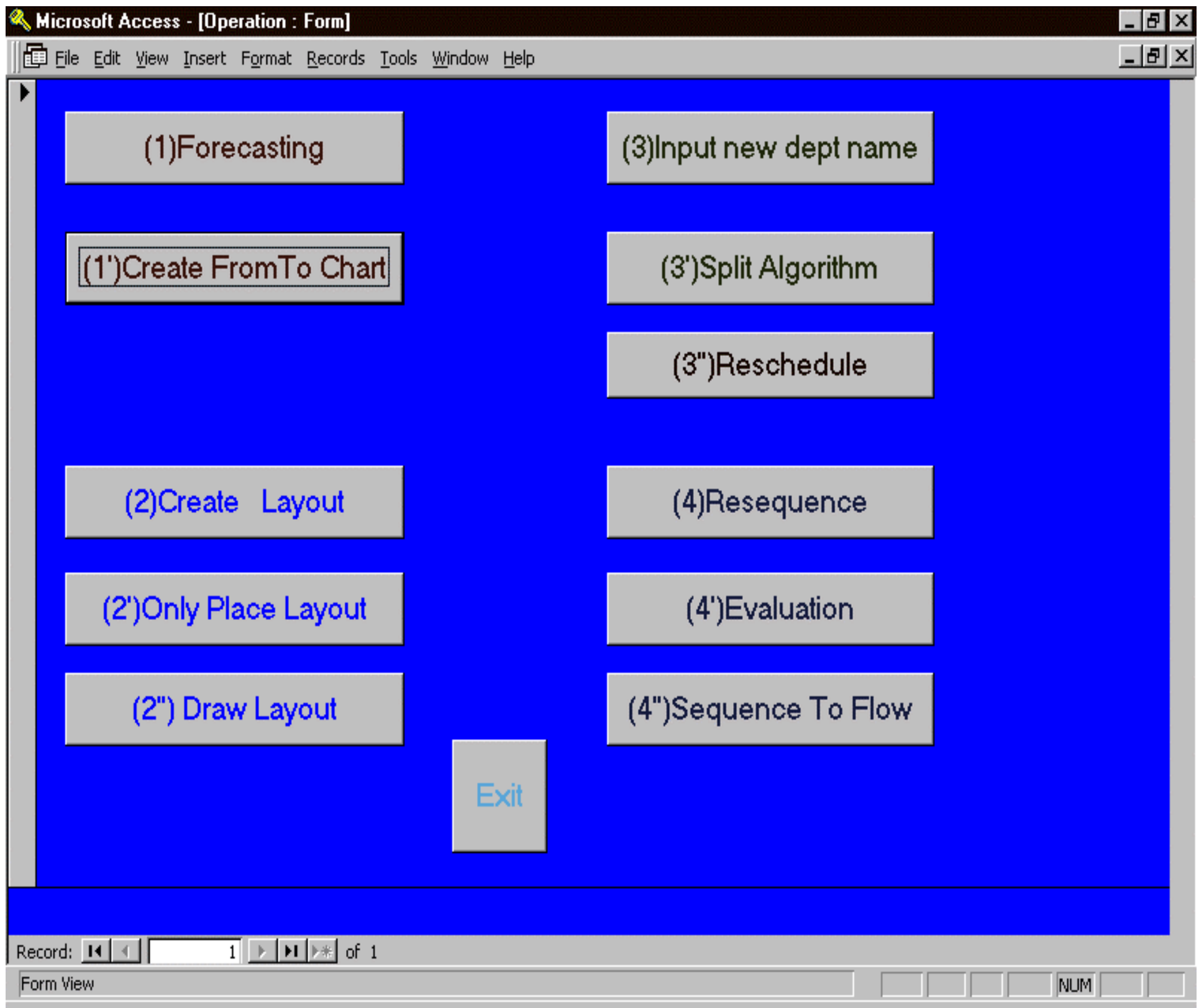




\section{APPENDIX - B. RELATIONSHIPS OF TABLES/QUERIES}

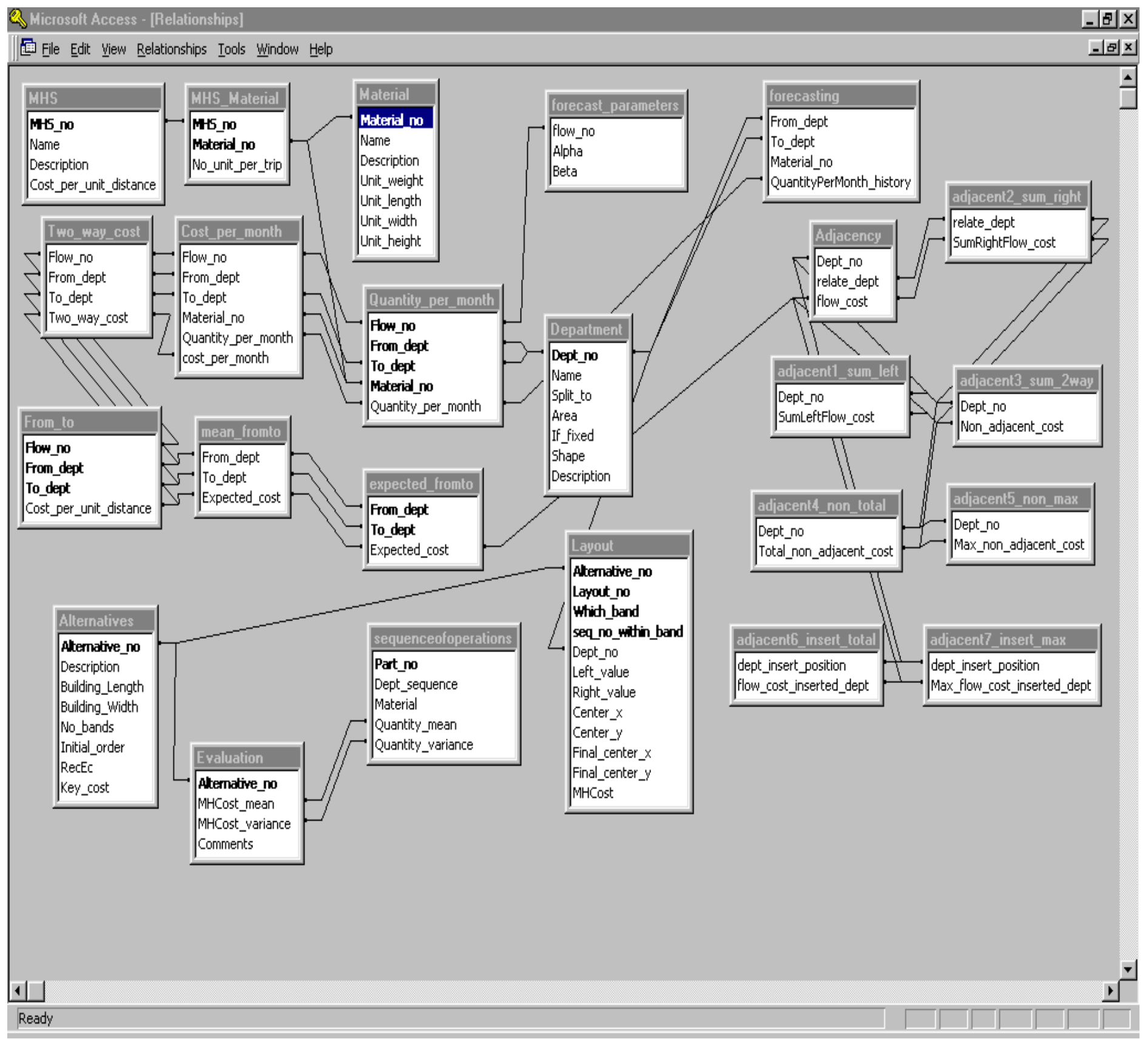




\section{APPENDIX - C. LIST OF ENTITIES}

\section{Table}

1) Adjacency

\begin{tabular}{|c|c|c|}
\hline Dept no & relate dept & flow cost \\
\hline
\end{tabular}

2) Alternatives

\begin{tabular}{|c|c|c|c|c|c|}
\hline Alternative_no & Description & Building_Length & Building_Width & No_bands & Initial order \\
\hline
\end{tabular}

3) Department

\begin{tabular}{|l|l|l|l|l|l|l|} 
Dept_no & Name & Split_to & Area & If_fixed & Shape & Description \\
\hline
\end{tabular}

4) Evaluation

\begin{tabular}{|l|l|l|l}
\hline Alternative_no & MHCost_mean & MHCost_variance & Comments
\end{tabular}

5) Expected_fromto

From_dept $\quad$ To_dept $\quad$ Expected_cost

6) Forecast_parameters
flow_no
Alpha
Beta

7) Forecasting

\begin{tabular}{|l|l|l|l|}
\hline From_dept & To_dept & Material_no & QuantityPerMonth_history \\
\hline
\end{tabular}

8) From_to

\begin{tabular}{|c|c|c|c|}
\hline Flow no & From dept & To dept & Cost per unit distance \\
\hline
\end{tabular}


9) Layout

\begin{tabular}{|l|l|l|l|l|l|l|}
\hline Alternative_no & Layout_no & Which_band & seq_no_within_band & Dept_no & Left_value & Right_value \\
\hline
\end{tabular} \begin{tabular}{|l|l|l|l|l|}
\hline Center_x & Center_y & Final_center_x & Final_center_y & MHCost \\
\hline
\end{tabular}

10) Material

\begin{tabular}{|l|l|l|l|l|l|l|}
\hline Material_no & Name & Description & Unit_weight & Unit_length & Unit_width & Unit_height \\
\hline
\end{tabular}

11) MHS

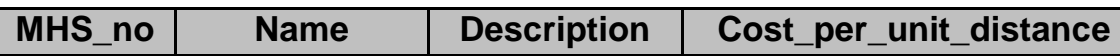

12) MHS_Material

MHS_no $\quad$ Material_no $\quad$ No_unit_per_trip

13) Quantity_per_month

\begin{tabular}{|l|l|l|l|l|} 
Flow_no & From_dept & To_dept & Material_no & Quantity_per_month \\
\hline
\end{tabular}

14) SequenceOfOperations

\begin{tabular}{|l|l|l|l|l|} 
Part_no & Dept_sequence & Material & Quantity_mean & Quantity_variance \\
\hline
\end{tabular}

15) Split_parameter

Max_percentage Max_no_portions

\section{Query}

1) Adjacent1_sum_left 


\section{Dept_no $\quad$ SumLeftFlow_cost}

2) Adjacent2_sum_right

relate_dept SumRightFlow_cost

3) Adjacent3_sum_2way

Dept_no $\quad$ Non_adjacent_cost

4) Adjacent4_non_total

Dept_no $\quad$ Total_non_adjacent_cost

5) Adjacent5_non_max

Dept_no $\quad$ Max_non_adjacent_cost

6) Adjacent6_insert_total

dept_insert_position $\quad$ flow_cost_inserted_dept

7) Adjacent7_insert_max

dept_insert_position $\quad$ Max_flow_cost_inserted_dept

8) Cost_per_month

\begin{tabular}{l|l|l|l|l|l|} 
Flow_no & From_dept & To_dept & Material_no & Quantity_per_month & cost_per_month \\
\hline
\end{tabular}

9) Mean_fromto

From_dept $\quad$ To_dept 1 Expected_cost

10) No_dept

No_dept 
11) No_fromtoRecord

No_FromtoRecord

12) Two_way_cost

\begin{tabular}{|l|l|l|l|}
\hline Flow_no & From_dept & To_dept & Two_way_cos \\
\hline
\end{tabular}




\section{APPENDIX - D. ENTITY INTERACTIONS}

\section{Table}

1) Adjacency

\begin{tabular}{|l|}
\hline \multicolumn{1}{|c|}{ Called by others } \\
\hline (Mod) Adjacent \\
\hline (Que) Adjacent1 \\
\hline (Que) Adjacent2 \\
\hline (Que) Adjacent6_insert_total \\
\hline
\end{tabular}

2) Alternatives

\begin{tabular}{|l|}
\hline \multicolumn{1}{|c|}{ Called by others } \\
\hline (Mod) PairExchange \\
\hline (Mod) Split_dept \\
\hline (Mod) Draw_layout \\
\hline (Mod) EvaluationLayout \\
\hline (Mod) Resequence \\
\hline (Mod) OnlyPlaceLayout \\
\hline (Mod) Reschedule_flow \\
\hline
\end{tabular}

3) Department

\begin{tabular}{|l|}
\hline \multicolumn{1}{|c|}{ Called by others } \\
\hline (Query) No_dept \\
\hline (Mod) PairExchange \\
\hline (Mod) Split_dept \\
\hline (For) For_dept \\
\hline (Mod) Resequence \\
\hline (Mod) OnlyPlaceLayout \\
\hline
\end{tabular}


(Mod) SequenceToFlow

(Mod) Draw_layout

(Mod) Reschedule_flow

4) Evaluation

\begin{tabular}{|c|}
\hline Called by others \\
\hline (Mod) EvaluationLayout \\
\hline
\end{tabular}

5) Expected_fromto

\begin{tabular}{|l|}
\hline \multicolumn{1}{|c|}{ Called by others } \\
\hline (Mod) Expected_fromto_queryToTable \\
\hline (Mod) PairExchange \\
\hline (Mod) CostPerDistance \\
\hline (Mod) Split_dept \\
\hline (Mod) OnlyPlaceLayout \\
\hline (Mod) Expected_fromto \\
\hline
\end{tabular}

6) Forecasting

\begin{tabular}{|c|}
\hline Called by others \\
\hline (Mod) Forecast \\
\hline
\end{tabular}

7) Forecast_parameters

\begin{tabular}{|l|}
\hline \multicolumn{1}{|c|}{ Called by others } \\
\hline (Mod) Forecast \\
\hline (Mod) SequenceToFlow \\
\hline
\end{tabular}

8) From_to

\begin{tabular}{|c|}
\hline Called by others \\
\hline (Mod) Fromto \\
\hline
\end{tabular}


(Que) No_fromtoRecord

(Que) Mean_fromto

9) Layout

\begin{tabular}{|l|}
\hline \multicolumn{1}{|c|}{ Called by others } \\
\hline (Mod) PairExchange \\
\hline (Mod) Adjacent \\
\hline (Mod) Split_dept \\
\hline (Mod) Draw_layout \\
\hline (Mod) EvaluationLayout \\
\hline (Mod) Resequence \\
\hline (Mod) OnlyPlaceLayout \\
\hline (Mod) Reschedule \\
\hline
\end{tabular}

10) MHS

\begin{tabular}{|l|}
\hline \multicolumn{1}{|c|}{ Called by others } \\
\hline (Que) Cost_per_month \\
\hline (Mod) EvaluationLayout \\
\hline
\end{tabular}

11) MHS_Material

\begin{tabular}{|l|}
\hline \multicolumn{1}{|c|}{ Called by others } \\
\hline (Que) Cost_per_month \\
\hline (Mod) EvaluationLayout \\
\hline
\end{tabular}

12) Quantity_per_month

\begin{tabular}{|l|}
\hline \multicolumn{1}{|c|}{ Called by others } \\
\hline (Que) Cost_per_month \\
\hline (Mod) Forecast \\
\hline (Mod) SequenceToFlow \\
\hline
\end{tabular}


13) SequenceOfOperations

\begin{tabular}{|l|}
\hline \multicolumn{1}{|c|}{ Called by others } \\
\hline (Mod) EvaluationLayout \\
\hline (Mod) Resequence \\
\hline (Mod) SequenceToFlow \\
\hline
\end{tabular}

14) [Material]

\section{Query}

1) Adjacent1_sum_left

\begin{tabular}{|l|l|}
\hline \multicolumn{1}{|c|}{ Call others } & \multicolumn{1}{c|}{ Called by others } \\
\hline (Tab) Adjacency & (Que) Adjacen3_sum_2way \\
\hline
\end{tabular}

2) Adjacent2_sum_right

\begin{tabular}{|l|c|}
\hline \multicolumn{1}{|c|}{ Call others } & Called by others \\
\hline (Tab) Adjacency & (Que) Adjacent3_sum_2way \\
\hline
\end{tabular}

3) Adjacent3_sum_2way

\begin{tabular}{|l|l|}
\hline \multicolumn{1}{|c|}{ Call others } & Called by others \\
\hline (Que) Adjacent1_sum_left & (Que) Adjacent4_non_total \\
\hline (Que) Adjacent2_sum_right & \\
\hline
\end{tabular}

4) Adjacent4_non_total

\begin{tabular}{|c|c|}
\hline Call others & Called by others \\
\hline (Que) Adjacent3_sum_2way & (Que) Adjacent5_non_max \\
\hline
\end{tabular}

5) Adjacent5_non_max 


\begin{tabular}{|c|c|}
\hline Call others & Called by others \\
\hline (Que) Adjacent4_non_total & (Que) Adjacent6_insert_total \\
\hline
\end{tabular}

6) Adjacent6_insert_total

\begin{tabular}{|l|l|}
\hline \multicolumn{1}{|c|}{ Call others } & \multicolumn{1}{c|}{ Called by others } \\
\hline (Que) Adjacent5_non_max & (Que) Adjacent7_insert_max \\
\hline (Tab) Adjacency & \\
\hline
\end{tabular}

7) Adjacent7_insert_max

\begin{tabular}{|c|c|}
\hline Call others & \multicolumn{1}{|c|}{ Called by others } \\
\hline (Que) Adjacent6_insert_total & (Mod) Split_dept \\
\hline
\end{tabular}

8) Cost_per_month

\begin{tabular}{|l|l|}
\hline \multicolumn{1}{|c|}{ Call others } & \multicolumn{1}{c|}{ Called by others } \\
\hline (Tab) Quantity_per_month & (Que) Two_way_cost \\
\hline (Tab) MHS_Material & \\
\hline (Tab) MHS & \\
\hline
\end{tabular}

9) Mean_fromto

\begin{tabular}{|l|l|}
\hline \multicolumn{1}{|c|}{ Call others } & \multicolumn{1}{c|}{ Called by others } \\
\hline (Tab) From_to & (Mod) Expected_fromto_queryToTable \\
\hline (Que) No_fromtoRecord & \\
\hline (Que) No_dept & \\
\hline
\end{tabular}

10) No_dept

\begin{tabular}{|l|l|}
\hline \multicolumn{1}{|c|}{ Call others } & \multicolumn{1}{c|}{ Called by others } \\
\hline (Tab) Department & (Que) Mean_fromto \\
\hline
\end{tabular}

11) No_FromtoRecord 


\begin{tabular}{|l|l|}
\hline \multicolumn{1}{|c|}{ Call others } & \multicolumn{1}{c|}{ Called by others } \\
\hline (Tab) From_to & (Que) Mean_fromto \\
\hline
\end{tabular}

12) Two_way_cost

\begin{tabular}{|c|l|}
\hline Call others & \multicolumn{1}{|c|}{ Called by others } \\
\hline (Que) Cost_per_month & (Mod) Fromto \\
\hline
\end{tabular}

\section{Form}

1) For_dept

\begin{tabular}{|l|l|}
\hline \multicolumn{1}{|c|}{ Call others } & \multicolumn{1}{c|}{ Called by others } \\
\hline (Tab) Department & (For) Operation (3) \\
\hline
\end{tabular}

2) Operation

\begin{tabular}{|l|l|}
\hline \multicolumn{1}{|c|}{ Buttons } & \multicolumn{1}{c|}{ Entities Called } \\
\hline (1) Forecasting & (Mod) Forecast \\
\hline (1') Create From-to chart & (Mod) Fromto \\
\hline (1') Create From-to chart & $\begin{array}{l}\text { (Mod) } \\
\text { Expected_fromto_queryToTable }\end{array}$ \\
\hline (2) Create Layout & (Mod) PairExchange \\
\hline (2') OnlyPlaceOneLayout & (Mod) OnlyPlaceLayout \\
\hline (2") Draw Layout & (Rep) Rep_layout \\
\hline (3) Input new dept name & (Mod) Adjacent \\
\hline (3) Input new dept name & (For) For_dept \\
\hline (3') Split Algorithm & (Mod) Split_dept \\
\hline (3") Reschedule & (Mod) Reschedule_flow \\
\hline (4) Resequence & (Mod) Resequence \\
\hline (4') Evaluation & (Mod) EvaluationLayout \\
\hline
\end{tabular}




\section{Report}

1) Rep_layout

\begin{tabular}{|c|c|}
\hline Call others & Called by others \\
\hline (Class Mod of (Rep) Rep_layout) Datail_print & (Mod) Draw_layout \\
\hline & (For) Operation (2") \\
\hline
\end{tabular}

\section{Module}

1) Mod_adjacent

\begin{tabular}{|l|l|}
\hline \multicolumn{1}{|c|}{ Call others } & \multicolumn{1}{c|}{ Called by others } \\
\hline (Tab) Layout & (For) Operation (3) \\
\hline (Mod) CostPerDistance & \\
\hline (Tab) Adjacency & \\
\hline
\end{tabular}

2) Mod_costPerDistance

\begin{tabular}{|c|l|}
\hline \multicolumn{1}{|c|}{ Call others } & \multicolumn{1}{c|}{ Called by others } \\
\hline (Tab) Expected_fromto & (Mod) Adjacent \\
\hline
\end{tabular}

3) Mod_draw_layout

\begin{tabular}{|l|c|}
\hline \multicolumn{1}{|c|}{ Call others } & Called by others \\
\hline (Tab) Layout & (Class Mod of (Rep) Rep_layout) Datail_print \\
\hline (Tab) Alternatives & \\
\hline (Tab) Department & \\
\hline (Rep) Rep_layout & \\
\hline
\end{tabular}


4) Mod_EvaluationLayout

\begin{tabular}{|l|l|}
\hline \multicolumn{1}{|c|}{ Call others } & \multicolumn{1}{c|}{ Called by others } \\
\hline (Tab) Layout & (For) Operation (4') \\
\hline (Tab) Alternatives & \\
\hline (Tab) SequenceOfOperations & \\
\hline (Tab) MHS_Material & \\
\hline (Tab) MHS & \\
\hline (Tab) Evaluation & \\
\hline
\end{tabular}

5) Mod_expected_fromto_queryToTable

\begin{tabular}{|l|l|}
\hline \multicolumn{1}{|c|}{ Call others } & \multicolumn{1}{c|}{ Called by others } \\
\hline (Que) Mean_fromto & (For) Operation (1') \\
\hline (Tab) Expected_fromto & \\
\hline
\end{tabular}

6) Mod_forecast

\begin{tabular}{|l|l|}
\hline \multicolumn{1}{|c|}{ Call others } & \multicolumn{1}{c|}{ Called by others } \\
\hline (Tab) Forecasting & (For) Operation (1) \\
\hline (Tab) Forecast_parameters & \\
\hline (Tab) Quantity_per_month & \\
\hline
\end{tabular}

7) Mod_fromto

\begin{tabular}{|l|l|}
\hline \multicolumn{1}{|c|}{ Call others } & \multicolumn{1}{|c|}{ Called by others } \\
\hline (Que) Two_way_cost & (For) Operation (1') \\
\hline (Tab) From_to & \\
\hline
\end{tabular}

8) Mod_locateDept

\begin{tabular}{|l|l|}
\hline Call others & \multicolumn{1}{|c|}{ Called by others } \\
\hline & (Mod) PairExchange \\
\hline
\end{tabular}

9) Mod_MHCost_calculate 


\begin{tabular}{|l|l|}
\hline Call others & \multicolumn{1}{|c|}{ Called by others } \\
\hline & (Mod) PairExchange \\
\hline
\end{tabular}

10) Mod_onlyPlaceLayout

\begin{tabular}{|l|l|}
\hline \multicolumn{1}{|c|}{ Call others } & \multicolumn{1}{c|}{ Called by others } \\
\hline (Tab) Expected_fromto & (For) Operation (2') \\
\hline (Tab) Alternatives & \\
\hline (Tab) OnlyPlaceLayout & \\
\hline (Tab) Layout & \\
\hline
\end{tabular}

11) Mod_pairExchange

\begin{tabular}{|l|l|}
\hline \multicolumn{1}{|c|}{ Call others } & \multicolumn{1}{c|}{ Called by others } \\
\hline (Mod) MHCost_calculate & (For) Operation (2) \\
\hline (Mod) LocateDept & \\
\hline (Tab) Expected_fromto & \\
\hline (Tab) Alternatives & \\
\hline (Tab) Department & \\
\hline (Tab) Layout & \\
\hline
\end{tabular}

12) Mod_reschedule_flow

\begin{tabular}{|l|l|}
\hline \multicolumn{1}{|c|}{ Call others } & \multicolumn{1}{c|}{ Called by others } \\
\hline (Tab) Layout & (For) Operation (3") \\
\hline (Tab) Alternatives & \\
\hline (Tab) Department & \\
\hline (Tab) Expected_fromto & \\
\hline
\end{tabular}

13) Mod_resequence

\begin{tabular}{|l|l|}
\hline \multicolumn{1}{|c|}{ Call others } & \multicolumn{1}{c|}{ Called by others } \\
\hline (Tab) Layout & (For) Operation (4) \\
\hline
\end{tabular}




\begin{tabular}{|l|l|}
\hline (Tab) Alternatives & \\
\hline (Tab) Department & \\
\hline (Tab) SequenceOfOperations & \\
\hline
\end{tabular}

14) Mod_SequenceToFlow

\begin{tabular}{|l|l|}
\hline \multicolumn{1}{|c|}{ Call others } & \multicolumn{1}{c|}{ Called by others } \\
\hline (Tab) SequenceOfOperations & (For) Operation (4”) \\
\hline (Tab) Forecast_parameters & \\
\hline (Tab) Department & \\
\hline (Tab) Quantity_per_month & \\
\hline
\end{tabular}

15) Mod_split_dept

\begin{tabular}{|l|l|}
\hline \multicolumn{1}{|c|}{ Call others } & \multicolumn{1}{c|}{ Called by others } \\
\hline (Que) Adjacent5_non_max & (For) Operation (3') \\
\hline (Que) Adjacent7_insert_max & \\
\hline (Tab) Expected_fromto & \\
\hline (Tab) Alternatives & \\
\hline (Tab) Department & \\
\hline (Tab) Layout & \\
\hline
\end{tabular}

16) (Class Module) Datail_print [of (REPORT) Rep_layout]

\begin{tabular}{|c|l|}
\hline \multicolumn{1}{|c|}{ Call others } & \multicolumn{1}{c|}{ Called by others } \\
\hline (Mod) Draw_layout & (Rep) Rep_layout \\
\hline
\end{tabular}

